\title{
Realização de conjunto de pontos fixos numa dada classe de homotopia equivariante de aplicações
}

\author{
Rafael Moreira de Souza
}

\author{
TESE APRESENTADA \\ $\mathrm{AO}$ \\ INSTITUTO DE MATEMÁTICA E ESTATÍSTICA \\ DA \\ UNIVERSIDADE DE SÃO PAULO \\ PARA \\ OBTENÇÃA DO TÍTULO \\ DE \\ DOUTOR EM CIÊNCIAS \\ Programa: Matemática \\ Orientador: Prof. Dr. Peter Ngai-Sing Wong \\ Coorientadora: Prof. ${ }^{\text {a }}$ Dr. ${ }^{\text {a }}$ Lucília Daruiz Borsari
}

Durante o desenvolvimento deste trabalho o autor recebeu auxílio financeiro da CAPES/FAPESP

São Paulo, junho de 2014 


\section{Realização de conjunto de pontos fixos numa dada classe de homotopia equivariante de aplicações}

Esta versão da tese contém as correções e alterações sugeridas pela Comissão Julgadora durante a defesa da versão original do trabalho, realizada em 29/05/2014.

Prof. Dr. Peter Ngai-Sing Wong (orientador) - Bates College

Prof. Dr. Daciberg Lima Gonçalves - IME-USP

Prof. Dr. Daniel Vendrúsculo - UfSCar

Prof ${ }^{\mathrm{a}}$. Dr ${ }^{\mathrm{a}}$. Thaís Fernanda Mendes Monis - UNESP

Prof. Dr. Weslem Liberato Silva - UESC 

Dedico este trabalho a você que o está lendo e espero que te ajude tanto quanto me ajudou. 


\section{Agradecimentos}

Agradeço a Deus por tudo que ocorre na minha vida, tanto pelas coisas ditas "boas" quanto pelas "ruins", pois acredito que tudo nessa vida é "remédio" para alguma coisa.

Agradeço a toda minha família, não só as pessoas maravilhosas com quem eu me divirto muito e que estão próximas o tempo todo que são meus pais, meus irmãos e suas esposas, minha afilhada linda, minha priminha Lili e minha namorada Kátia Carolino e sua família, mas aos meus tios, primos, avôs,... pessoas vivas ou mortas que amo e que acrescentaram alguma coisa em mim que foi útil nesse trabalho.

Agradeço aos professores que tive, tanto os que colaboraram em minha formação de base, que hoje ingratamente não me lembro do nome de todos, quanto os mais recentes que tive em minha graduação e pós-graduação. Em especial ao meu orientador Professor Dr. Peter Ngai-Sing Wong que criou esse projeto, me aceitou como orientado, esteve sempre disponível para me ajudar, me recebeu muito bem em Lewiston, me deu várias dicas com relação a minha área de estudo e que contribuiu com várias observações relevantes relacionadas a elaboração deste texto e a minha co-orientadora Professora Dr. ${ }^{a}$ Lucília Daruiz Borsari que aceitou me co-orientar, me aconselhou muito e de forma muito educada, cuidadosa e elegante, esteve sempre presente e disponível contribuindo muito com o meu aprendizado dos pré-requisitos e com a elaboração de todos os textos que apresentei durante o doutorado.

Outros professores que merecem um agradecimento especial são o Professor Dr. Daciberg Lima Gonçalves que muito me ajudou com relação a FAPESP, sempre esteve disponível e agindo com muita solicitude, o Professores Dr. Daniel Vendrúsculo que me orientou desde a graduação até o fim do mestrado, me enviou para São Paulo, me apresentou o Professor Peter Wong e que com muito carinho me refiro por "Boss" , o professor Dr. Ivo Machado da Costa pela amizade e interesse e ao Professor Dr. Roberto Ribeiro Paterlini sem o qual eu teria desistido da matemática há muito tempo. Sei que não sou sempre fácil ou dócil o que aumenta o crédito de todas essas pessoas. Todos me ensinaram algo.

Agradeço aos meus amigos, presentes ou ausentes, próximos ou distantes, os de infância ou adolescência e os mais recentes que estão no começo de minha vida adulta. Pessoas que poderiam ser mais um transeunte mas que Deus caprichosamente os inseriu, cada um em seu momento, em minha vida.

Agradeço o apoio financeiro da CAPES, da FAPESP, o suporte técnico da Bates College e do Instituto de Matemática e Estatística (IME) da USP, a Superintendência de Assistência Social (SAS) da USP e a todos que colaboraram, direta ou indiretamente, na 
concretização deste trabalho.

Por fim, agradeço as pessoas, tão pouco reconhecidas, que tornaram minha vida mais fácil durante esses anos. Falo das faxineiras, dos porteiros, dos pedreiros e seus ajudantes, dos jardineiros, dos seguranças,... funcionários que vivem em seu dia-a-dia a realidade do salário mínimo, o descaso do transporte público, a alienação da educação pública de base e a lentidão da saúde pública. 


\section{Resumo}

Souza, R. M. Realização de conjunto de pontos fixos numa dada classe de homotopia equivariante de aplicações. 2014, 102f. Tese - Instituto de Matemática e Estatística, Universidade de São Paulo, São Paulo, 2014.

Nesse trabalho combinamos a teoria de Nielsen de pontos fixos com a teoria dos grupos de transformações para dar condições necessárias e suficientes para realizar um $G$ subconjunto $A$ localmente contrátil de $X$ como o conjunto de pontos fixos de uma aplicação equivariante $h: X \rightarrow X$ em uma classe de homotopia equivariante dada, onde $G$ é um grupo de Lie compacto e $X$ é uma $G$-variedade suave e compacta.

Além disso, se $X$ é o espaço total de um $G$-fibrado localmente trivial demos condições necessárias e suficientes para o correspondente problema de realização para aplicações $G$-equivariantes que preservam fibra, onde $G$ é um grupo finito. 


\section{Abstract}

Souza, R. M. Realization of fixed point set in a prescribed equivariant homotopy class of maps. 2014, 102f. Thesis - Instituto de Matemática e Estatística, Universidade de São Paulo, São Paulo, 2014.

In this work, we combine the Nielsen fixed point theory with the transformation group theory to present necessary and sufficient conditions for the realization of a locally contractible $G$-subset $A$ of $X$ as the fixed point set of a map $h: X \rightarrow X$ in a given $G$-homotopy class. Here, $G$ is a compact Lie group and $X$ is a compact smooth $G$-manifold.

In adition, if $X$ is the total space of a $G$-fiber bundle we present necessary and sufficient conditions for the corresponding realization problem for $G$-fiber-preserving maps when $G$ is a finite group. 


\section{Sumário}

Introdução $\quad$ xiii

1 Preliminares 1

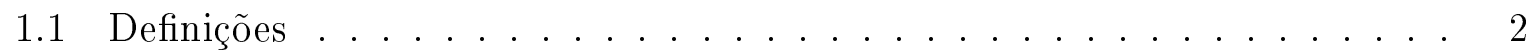

1.1.1 Definições Gerais . . . . . . . . . . . . . . . . . . . 2

1.1 .2 Complexos Simpliciais . . . . . . . . . . . . . . . 3

1.1.3 Fibrados e Fibrações . . . . . . . . . . . . . . . . . . . . . . . 6

1.1.4 Classes de Nielsen e Índice de Pontos Fixos . . . . . . . . . . . . . . 8

1.1.5 Realização de um Conjunto como Conjunto de Pontos Fixos de uma dada Aplicação . . . . . . . . . . . . . . . . . . . . . . 11

1.2 Definições Relativas aos Grupos de Transformações . . . . . . . . . . . . . 15

1.2 .1 G-espaços e $G$-aplicações . . . . . . . . . . . . . . . . . . . 15

1.2 .2 Índice de Pontos Fixos Equivariante . . . . . . . . . . . . . . . . . . 18

1.2 .3 Aplicações que Preservam Fibra . . . . . . . . . . . . . . . . . . 18

1.3 Resultados da Bibliografia . . . . . . . . . . . . . . . . . . . 20

1.3.1 Resultados da Bibliografia para Espaços e Aplicações . . . . . . . . 20

1.3.2 Resultados da Bibliografia para Fibrados e Fibrações . . . . . . . . 25

2 Realização de Conjuntos de Pontos Fixos 28

2.1 Exemplos . . . . . . . . . . . . . . . . . . . . . . 29

2.2 Resultados Preliminares . . . . . . . . . . . . . . . . . . 34

2.3 Procedimentos em $G$-Classes de Pontos Fixos . . . . . . . . . . . . . . 38

2.4 Realização de um Conjunto como Conjunto de Pontos Fixos de uma dada G-Aplicação . . . . . . . . . . . . . . . . . 46

3 Pontos Fixos de Aplicações que Preservam Fibra $\quad 54$

3.1 Exemplos Relacionados a Aplicações que Preservam Fibra . . . . . . . . . 55

3.2 Resultados Preliminares para Aplicações que Preservam Fibra . . . . . . . 58

3.3 Realização de um Conjunto como Conjunto de Pontos Fixos de uma dada G-Aplicação que Preserva Fibra . . . . . . . . . . . . . . . 64

$\begin{array}{ll}\text { A Retração Local } & 73\end{array}$ 
B Fibrado vs Fibração 


\section{Lista de Figuras}

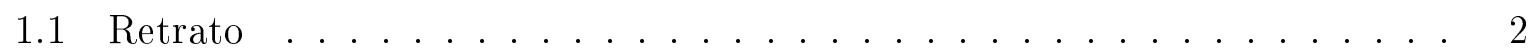

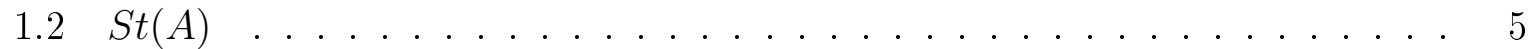

1.3 Aplicação Proximal . . . . . . . . . . . . . . . . . . . 5

1.4 Preservar Fibra . . . . . . . . . . . . . . . . . . . . . . 6

1.5 Fibração . . . . . . . . . . . . . . . . . . . . . . . 7

1.6 Preservar Fibra . . . . . . . . . . . . . . . . . . . . . . . . . 8

2.1 Coordenadas Esféricas . . . . . . . . . . . . . . . . . . . . . . . . . 29

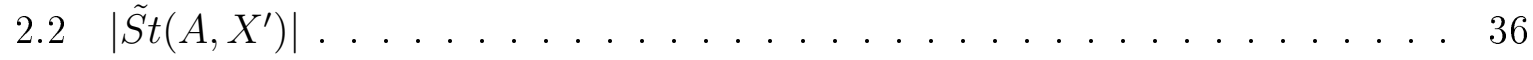

2.3 A e $Y$ contornáveis . . . . . . . . . . . . . . . . 37

2.4 Interseções . . . . . . . . . . . . . . . . . . . . . . . . . . . . . . . 39

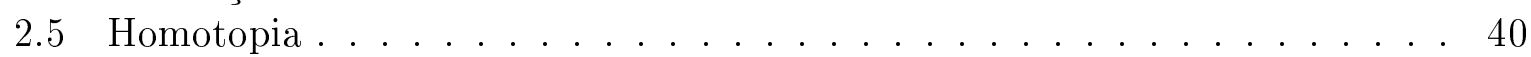

$2.6 U(s(I)) \ldots \ldots \ldots \ldots \ldots \ldots \ldots \ldots$

2.7 Lema VIII.C.3 . . . . . . . . . . . . . . . . . . . . . . . 44

2.8 Vizinhança $V \ldots \ldots \ldots \ldots$

A.1 Plano $\pi \ldots \ldots \ldots \ldots \ldots \ldots \ldots \ldots$ 


\section{Lista de Símbolos}

$\begin{array}{ll}\bar{A}, 2 & \Lambda, 8 \\ \partial A, 2 & \Omega_{p}, 8,15 \\ (X, A), 2,17 & I_{f}, 9,10 \\ \operatorname{Int}(\mathrm{A}), 2 & I_{\left.f\right|_{U}}, 11 \\ \mathrm{I}, 2 & I(f, N), 11 \\ \approx, 2 & \mathcal{N}(f), 11 \\ \text { rel, } 2 & \mathrm{NK}, 15 \\ X^{I}, 3 & \left(K_{1}\right) \leq\left(K_{2}\right), 16 \\ \mathbb{R}^{n}, 4 & \mathrm{Iso}(\mathrm{X}), 16 \\ |K|, 4 & X^{K}, 16 \\ |s|, 4 & X^{(K)}, 16 \\ \sigma(x), 4 & X_{K}, 16 \\ \mathrm{St}(\mathrm{v})(\text { onde v é um vértice }), 4 & \mathrm{WK}, 16 \\ \mathrm{St}(\mathrm{t})(\text { onde t é um simplexo), } & G_{x}, 16 \\ \mathrm{St}(\mathrm{A})(\text { onde A é um subconjunto }), 5 & X_{(K)}, 16 \\ {[a, b], 5} & (\mathrm{~K}), 16 \\ \mathfrak{F}=(X, p, B, Y), 6 & X_{i}, 16 \\ \mathfrak{F}^{\prime}=\left(A, p, B, Y_{0}\right), 6 & C^{\infty}, 17 \\ \left.\mathfrak{F}\right|_{W}=(p-1(W), p, W, Y), 7 & \bar{f}, 19 \\ \mathbb{S}^{n}, 8 & G(x), 22 \\ F i x(f), 8 & \frac{G}{H}, 22\end{array}$




\section{Introdução}

Uma das questões clássicas no estudo de pontos fixos é a propriedade do ponto fixo, isso é, dado um espaço topológico $X$, determinar se qualquer aplicação $f: X \rightarrow X$ obrigatoriamente possui um ponto fixo. De um tal espaço diz-se ter a propriedade do ponto fixo. Usando topologia algébrica pode-se facilmente mostrar que o disco fechado $D^{n}$ em $\mathbb{R}^{n}$ possui a propriedade do ponto fixo.

Uma questão relacionada é indagar se um espaço possui a propriedade de invariância completa para deformações (PICD). Um espaço topológico $X$ possui a PICD se para qualquer subconjunto fechado não vazio $A$ de $X$, existir uma aplicação $f: X \rightarrow X$ homotópica a identidade em $X$ tal que $A=F i x f$, o conjunto de pontos fixos de $f$. Substituindo a identidade em $X$ por uma aplicação $f$ fixada, a alteração apropriada para a questão anterior seria se um subconjunto fechado não vazio $A$ pode ser realizado como o conjunto de pontos fixos de alguma aplicação $f_{1}$ homotópica a $f$ (veja $[9,10]$ ).

Em 1990, H. Schirmer fez uso da teoria de Nielsen de pontos fixos [11] para dar condições necessárias e suficientes sobre subpoliedros não vazios para que pudessem ser realizados como o conjunto de pontos fixos de uma aplicação em uma classe de homotopia de uma autoaplicação dada [9]. Na realidade, o artigo [9] corrigiu uma hipótese incorreta que se pensava ser suficiente como afirmava P. Strantzalos in 1977.

Subsequentemente, generalizações do resultado de Schirmer foram obtidos. Em particular, C. Soderlund [20] ampliou o resultado de Schirmer assumindo que $A$ era localmente contrátil, e obteve também uma versão relativa do teorema de Schirmer para aplicações de pares, estendendo o trabalho de Ng [21]. Mais recentemente, R. Brown e C. Soderlund [19] generalizaram o resultado de Schirmer no contexto de aplicações que preservam fibras.

Muitas aplicações envolvem simetrias como um resultado da ação de um grupo. Questões topológicas para aplicações e espaços envolvendo ações de grupos são particularmente ricas e complexas. No caso equivariante, preocupa-se com um grupo $G$ (finito) atuando sobre um espaço $X$ munido de uma aplicação $G$-equivariante $f: X \rightarrow X$ que respeita a ação do grupo, isso é , para todo $\alpha \in G, f(\alpha x)=\alpha f(x)$ para todo $x \in X$.

Nesse caso, o conjunto de pontos fixos Fixf é a priori um subconjunto $G$-invariante de $X$. O estudo topológico da teoria de pontos fixos para aplicações equivariantes $\grave{a}$ la Nielsen começou com [12] e subsequentemente em [13, 14, 15, 16]. Usando as ideias da teoria de pontos fixos de Nielsen equivariante, uma versão equivariante da PICD em $G$-variedades foi dada por P. Wong [14] onde $G$ é grupo finito atuando suavemente em variedades. 
Combinando os resultados de [9] com o contexto equivariante, a questão natural, e objeto de estudo deste trabalho, é determinar condições necessárias e suficientes para que um subconjunto fechado não vazio $G$-invariante $A$ seja o conjunto de pontos fixos de uma aplicação equivariante em uma dada classe de $G$-homotopia de uma dada aplicação $G$-equivariante.

As respostas que encontramos foram dadas nos capítulos dois e três, sempre trabalhando com variedades no contexto equivariante. No capítulo um estabelecemos uma notação padrão para todo o trabalho. Além disso, apresentamos com mais detalhes os trabalhos [9] de H. Schirmer e [19] de R. Brown e C. L. Soderland no final da primeira seção, e nas duas seções seguintes abordamos os elementos da teoria dos grupos de transformações que usaremos e todos os resultados que faremos referência nos dois capítulos seguintes.

No capítulo dois generalizamos o Lema 3.1 de [9] para o contexto equivariante, e usaremos a ideia apresentada por C. L. Soderlund em [20] que possibilita realizar subconjuntos localmente contráteis como o conjunto de pontos fixos de uma aplicação dada. Os principais resultados encontrados no segundo capítulo são:

Teorema 2.10 (pg 46): Sejam $G$ um grupo de Lie compacto, $X$ uma $G$-variedade suave e compacta, $A$ um $G$-subconjunto de $X$ localmente contrátil, fechado e não vazio e $f: X \rightarrow X$ uma $G$-aplicação tais que para $W K$ finito temos que $\operatorname{dim}\left(X^{K}\right) \geq 3$, $\operatorname{dim}\left(X^{K}\right)-\operatorname{dim}\left(X^{K}-X_{K}\right) \geq 2$ e $A^{K}$ é contornável em $X^{K}$, para todo $(K) \in I s o(X)$.

Então $f$ é $G$-homotópica a uma $h: X \rightarrow X$ tal que $F i x(h)=A$ se, e somente se, as seguintes condições estiverem satisfeitas:

(C1) existe uma $G$-aplicação $\bar{H}:(X \times\{0\}) \cup(A \times I) \rightarrow X$ tal que $\bar{H}(\bullet, 0)=f$ e $\bar{H}(\bullet, 1)=i: A \hookrightarrow X$ (inclusão);

(C2) sempre que $W K$ for finito, toda $W K$-classe de pontos fixos essencial $F$ de $f^{K}$ está conectada a $A^{K}$ por um caminho $p: I \rightarrow X^{K}\left(p(0) \in F\right.$ e $\left.p(1) \in A^{K}\right)$ tal que $\{p(t)\}$ é homotópica à $\left\{f^{K} \circ p\right\} *\left\{\bar{H}^{K}(p(1), t)\right\}$ com pontos extremos fixados.

Corolário 2.16 (pg 51): Sejam $G$ um grupo finito, $X$ uma $G$-variedade suave e compacta com $\operatorname{dim}\left(X^{K}\right) \geq 3$ e $\operatorname{dim}\left(X^{K}\right)-\operatorname{dim}\left(X^{K}-X_{K}\right) \geq 2$ e $\Phi$ um $G$-subconjunto fechado, não vazio e localmente contrátil de $X$ tais que $\Phi^{K}$ é contornável em $X^{K}$, onde $(K) \in I \operatorname{so}(X)$.

Sejam $A$ um $G$-subconjunto fechado e não vazio de $\Phi$ que intersecta todas as componentes conexas de $\Phi$ e $f: X \rightarrow X$ uma $G$-aplicação. Então $f$ é $G$-homotópica a uma $h: X \rightarrow X$ tal que $F i x(h)=A$ se as seguintes condições estiverem satisfeitas:

(C1) existe uma $G$-aplicação $\bar{H}:(X \times\{0\}) \cup(\Phi \times I) \rightarrow X$ tal que $\bar{H}(\bullet, 0)=f$ e $\bar{H}(\bullet, 1)=i: \Phi \hookrightarrow X$ (inclusão). 
(C2) toda $W K$-classe de pontos fixos essencial $F$ de $f^{K}$ está conectada a $\Phi^{K}$ por um caminho $p: I \rightarrow X^{K}\left(p(0) \in F\right.$ e $\left.p(1) \in \Phi^{K}\right)$ tal que $\{p(t)\}$ é homotópica à $\left\{f^{K} \circ p\right\} *\left\{\bar{H}^{K}(p(1), t)\right\}$ (com pontos extremos fixados).

O artigo [19] traz condições necessárias e suficientes para realizar um conjunto localmente contrátil como conjunto de pontos fixos de uma dada aplicação entre fibrados que preserva fibra, generalizando os resultados de [9] para o contexto dos fibrados e aplicações que preservam fibra. No terceiro capítulo generalizamos essas condições para o contexto equivariante e o principal resultado encontrado é o

Teorema 3.11 (pg 70): Dados $G$ um grupo finito, $\mathfrak{F}=(X, p, B, Y)$ um $G$-fibrado onde $X, B$ e $Y$ são $G$-variedades suaves e compactas, $\operatorname{dim}\left(B^{K}\right) \geq 3, \operatorname{dim}\left(B^{K}\right)-\operatorname{dim}\left(B^{K}-\right.$ $\left.B_{K}\right) \geq 2$, para $(K) \in I s o(B)$, e $\operatorname{dim}\left(Y^{K}\right) \geq 3$ e $\operatorname{dim}\left(Y^{K}\right)-\operatorname{dim}\left(Y^{K}-Y_{K}\right) \geq 2$, para $(K) \in I s o(Y)$.

Seja $A$ um $G$-subconjunto não vazio, fechado e localmente contrátil de $X$ tal que $(X, A)$ é um $G$-par de fibrados para $\mathfrak{F}, p(A)$ é um $G$-subconjunto fechado de $B$, cada componente $p(A)_{j}$ de $p(A)$ se contrai equivariantemente em um ponto $b_{j} \in p(A)_{j}, p^{K}\left(A^{K}\right)$ é contornável em $B^{K}$, para $(K) \in I s o(B)$.

Supondo que $f: X \rightarrow X$ é uma $G$-aplicação que preserva fibra, que para todo subfibrado $Y_{j}$ de $A$ temos que $Y_{j}$ é um $G$-subconjunto fechado, localmente contrátil de $Y$ e $Y_{j}^{K}$ é contornável em $Y^{K}$, para todo $(K) \in I s o(Y)$, e que $A^{K}$ intercepta todas as $W K$-classes de pontos fixos essenciais de $f_{b_{j}}^{K}: W K\left(p^{K}\right)^{-1}\left(b_{j}\right) \rightarrow W K\left(p^{K}\right)^{-1}\left(b_{j}\right)$ para pelo menos um $b_{j}$ na componente $p^{K}\left(A^{K}\right)_{j}$, para todo $(K) \in I s o(X)$.

Se $Z$ é um $G$-subconjunto fechado e fibrado de $A$ que intercepta todas as componentes de $A$, então, existe uma $G$-aplicação $g: X \rightarrow X$ que é $G$-homotópica a $f$ por uma homotopia que preserva fibra tal que $F i x(g)=Z$ se as condições seguintes condições forem válidas:

$(C 1)_{\mathfrak{F}}$ existe uma $G$-homotopia parcial que preserva fibra $H_{A}:(X \times\{0\}) \cup(A \times I) \rightarrow X$ tal que $H_{A}(\bullet, 0)=f$ e $H_{A}(\bullet, 1)=i: A \hookrightarrow X$ (inclusão);

$(C 2)_{\mathfrak{F}}$ toda $W K$-classe de pontos fixos essencial $F$ de $f^{K}$ está conectada a $A^{K}$ por um caminho $\gamma: I \rightarrow X^{K}$ tal que $\gamma(0) \in F, \gamma(1) \in A^{K}$ e $\{\gamma(t)\}$ é homotópica à $\left\{f^{K} \circ \gamma\right\} *\left\{H_{A}^{K}(\gamma(1), t)\right\}$ com pontos extremos fixados. 


\section{Capítulo 1}

\section{Preliminares}

O objetivo desse trabalho é apresentar condições necessárias e suficientes para que um subconjunto fechado não vazio $G$-invariante $A$ seja o conjunto de pontos fixos de uma aplicação equivariante em uma dada classe de $G$-homotopia de uma dada aplicação $G$ equivariante, e o caminho escolhido foi combinar os resultados de [9] e de [19] com o contexto equivariante.

Nesse sentido, foi necessário consultar várias referências relativas a teoria dos grupos de transformações e a teoria de ponto fixo de Nielsen encontradas em [9] e em [19]. Cada texto trazia suas próprias notações e muitas vezes definições com significados diferentes entre si. Assim, coube a esse prólogo unificar as notações e reunir os resultados encontrados nas referências que serão usados nas próximas partes do texto para que o trabalho fique o mais padronizado e autocontido possível.

O primeiro capítulo desse texto está dividido em três seções, cada uma delas contendo um breve compêndio auto-explicativo relacionado ao seu tema. No primeiro estão as notações e definições dos objetos não equivariantes usados para exprimir as ideias usadas por H. Schirmer em [9] e por R. F. Brown e C. L. Soderlund em [19], e termina com uma breve prova dos resultados que generalizamos. O segundo abrange as notações e definições dos objetos equivariantes que serão trabalhados nos capítulos 2 e 3 . Por fim, o terceiro é um catálogo dos resultados tirados da bibliografia. 


\subsection{Definições}

Como mencionado anteriormente, faremos na primeira seção desse primeiro capítulo um compêndio com notações e definições dos objetos não equivariantes usados para exprimir as ideias usadas em $[9,19]$.

É importante citar que começaremos aqui a estabelecer uma linguagem padrão que será utilizada até o final deste trabalho. Para que os objetos que apresentaremos não fiquem espalhados de forma desorganizada quebraremos essa seção, e as duas seguintes, em subseções.

\subsubsection{Definições Gerais}

Adotaremos o termo aplicação quando estivermos tratando de uma função que é no mínimo contínua entre espaços topológicos.

Diremos que $(X, A)$ é um par de espaços se $X$ for um espaço topológico e $A \subset X$ um subespaço de $X$. Assim, dado um par topológico $(X, A)$ diremos que um subconjunto $V$ de $X$ é uma vizinhança de $A$ em $X$ se $A \subset V, V$ será chamado de vizinhança aberta (fechada) de $A$ em $X$ se $V$ for aberto (fechado) em $X$. Indicaremos por $\bar{A}$ o fecho de $A$

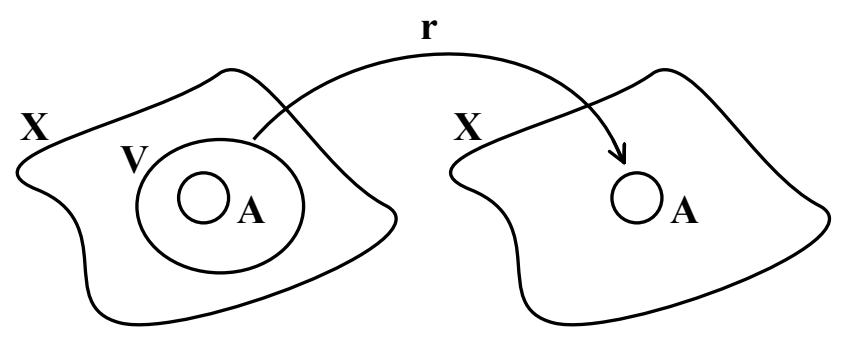

Figura 1.1: Retrato em $X$, por $\partial(A)$ o bordo de $A$ em $X$ e por $\operatorname{Int}(A)$ o interior de $A$ em $X$.

Daí, dado um subespaço $V$ de $X$ que contém $A$ diremos que uma aplicação $r: V \rightarrow A$ é uma retração se a restrição $\left.r\right|_{A}: A \rightarrow A$ for igual a identidade, isto é, $\left.r\right|_{A}(a)=I d_{A}(a)=a$ para todo $a \in A$.

Quando $V$ for um aberto de $X$ chamaremos $A$ de um retrato de uma vizinhança de $X$ e diremos que o aberto $V \subset X$ se retrai sobre $A$. Em todo esse trabalho denotaremos por $I$ o intervalo fechado $[0,1]$, assim, diremos que $A$ é um retrato forte de uma vizinhança de $X$ quando existir uma homotopia $H: V \times I \rightarrow V$ entre $i \circ r$ e a identidade em $V$ relativamente a $A\left(i \circ r \approx I d_{V} \operatorname{rel} A\right)$, isto é, $H(x, 0)=x$ para todo $x \in V$, $H(x, t)=x$ para todo $(x, t) \in A \times I$ e $H(x, 1)=i \circ r(x)$ para todo $x \in V$, onde $i: A \hookrightarrow V$ é a inclusão e $r: V \rightarrow A$ é a retração. Nesse casso, diremos que a homotopia $H$ é relativa a $A$.

Quando $X$ for um espaço métrico com métrica $d$ temos que uma homotopia $H$ : $X \times I \rightarrow X$ é uma $\epsilon$-homotopia se para todo $t \in I$ tivermos que:

$$
\sup _{t, t^{\prime} \in I}\left\{d\left(H(x, t), H\left(x, t^{\prime}\right)\right) ; x \in X\right\}<\epsilon .
$$

Além disso, o espaço métrico $X$ é um ANR se, e somente se, existe uma aplicação injetiva $i: X \hookrightarrow Y$, onde $Y$ é um espaço métrico separável, tal que $i(X)$ é um retrato de uma 
vizinhança de $Y$. Usaremos a palavra mergulho como sinônimo para aplicação injetora. Assim $i$ é um mergulho.

Um espaço métrico $X$ é um ENR se, e somente se, existe um mergulho $i: X \rightarrow E$ de $X$ em um espaço euclidiano $E$ tal que $i(X)$ é um retrato de uma vizinhança de $E$. Dessa forma, a diferença entre os espaços ANR e ENR é que o espaço métrico separável imposto pelos ANR é substituído por um espaço euclidiano nos ENR, o que torna todo espaço ENR um espaço ANR.

Um caminho em um espaço topológico $X$ é qualquer aplicação $\gamma: I \rightarrow X$. Dado um par de espaços $(X, A)$ diremos que $A$ é contornável com relação a $X$ se todo caminho $\gamma: I \rightarrow X$ em $X$ tal que $\gamma(0), \gamma(1) \in X-A$ for homotópico a um caminho $\gamma^{\prime}: I \rightarrow X$ tal que $\gamma^{\prime}(I) \subset X-A$. Uma outra construção feita com caminhos é o espaço dos caminhos. Dado um espaço topológico $X$ tomemos $\alpha \in X^{I}=\{\gamma: I \rightarrow X ; \gamma$ contínua $\}$, daí, diremos que $X^{I}$ com a topologia compacto-aberto é o espaço dos caminhos de $X$.

Diremos que um ponto $x \in X$ de um espaço topológico $X$ é um ponto de corte local se existir um aberto $V$ de $X$ tal que $x \in V$ e $V-\{x\}$ não é conexo. Dessa forma, a existência, ou não, de pontos de corte local é uma propriedade relacionada com a geometria de $X$. Observamos que todos os pontos da esfera $S^{1}$ são pontos de corte local e que o cilindro $S^{1} \times I$ não possui nenhum pontos de corte local.

Dado um espaço topológico $X$ diremos que $X$ é localmente contrátil se toda vizinhança aberta $V_{x}$ de um ponto $x \in X$ contém uma vizinhança aberta $W_{x}$ de $x$ em $V_{x}$ que se contrai em $x$ e diremos que $X$ é localmente compacto se toda vizinhança aberta $A_{x}$ de um ponto $x \in X$ contém uma vizinhança compacta $K_{x} \subset A_{x}$ de $x$.

\subsubsection{Complexos Simpliciais}

As definições e notações dessa subseção seguem principalmente, mas não unicamente, de $[1,2,8]$. Um complexo simplicial $K$ é uma coleção finita de conjuntos finitos tal que se $s \in K$ e $t \subset s$ então $t \in K$.

Um conjunto $s \in K$ será chamado de simplexo de $K$, mais especificamente, se $s$ possuir $(\mathrm{p}+1)$ elementos diremos que $s$ é um p-simplexo de $K$. Os 0 -simplexos serão chamados de vértices. Um subcomplexo $L$ de $K$ é um complexo simplicial $L$ que é uma subcoleção de $K$.

Diremos que um $n$-simplexo $s$ de $K$ é maximal em $K$ se $K$ não possuir nenhum $m$-simplexo $t$, com $m>n$, tal que $s \subset t$. Daí, se não existir nenhum $m$-simplexo $t$ de $K$ tal que $m>n$ diremos que a dimensão de $K$ é $n$ e denotaremos isso por $\operatorname{dim}(K)=n$. Dados dois simplexos $s$ e $t$ de $K$ tais que $s \subset t$ diremos que $s$ é uma face de $t$, e se $t$ for um $n$-simplexo e $s$ um $(n-1)$-simplexo então diremos que $s$ é uma face maximal de $t$.

Dados dois complexos simpliciais $K$ e $L$ diremos que uma função $f: K \rightarrow L$ é simplicial se $f$ levar os simplexos de $K$ em simplexos de $L$, isto é, dado um n-simplexo $\left\{v_{0}, \ldots, v_{n}\right\}$ de $K$ temos que:

$$
f\left(\left\{v_{0}, \ldots, v_{n}\right\}\right)=\left\{f\left(v_{0}\right), \ldots, f\left(v_{n}\right)\right\} \in L .
$$


Notemos que $\left\{f\left(v_{0}\right), \ldots, f\left(v_{n}\right)\right\}$ é necessariamente um simplexo de $L$ mas não é necessário que seja um n-simplexo, pois pode ocorrer que existam $i<j \in\{0, \ldots, n\}$ tais que $f\left(v_{i}\right)=f\left(v_{j}\right)$.

Podemos agregar uma estrutura geométrica oriunda de um espaço euclidiano a um complexo simplicial $K$. De fato, sejam $\left\{v_{1}\right\},\left\{v_{2}\right\}, \ldots,\left\{v_{n}\right\}$ os vértices de $K$, segue que existe uma correspondência entre os vértices $\left\{v_{i}\right\}$ e os vetores $e_{i}$ da base canônica do espaço euclidiano $\mathbb{R}^{n}$. Consideremos $\Psi$ o conjunto de todas as combinações lineares $\sum_{i=1}^{n} r_{i} v_{i}$ que satisfazem as seguintes condições:

1. $r_{i}$ é um número real e $r_{i} \geq 0$ para $i=1, \ldots, n$;

2. $\sum_{i=1}^{n} r_{i}=1$;

3. $\left\{v_{i} \mid r_{i} \neq 0\right\} \in K$.

A métrica que usaremos em $\Psi$ é dada por:

$$
\begin{aligned}
& d: \quad \Psi \times \Psi \quad \rightarrow \quad \mathbb{R} \\
& \left(\sum_{i=1}^{n} r_{i} v_{i}, \sum_{i=1}^{n} t_{i} v_{i}\right) \mapsto\left(\sum_{i=1}^{n}\left(r_{i}-s_{i}\right)^{2}\right)^{\frac{1}{2}} .
\end{aligned}
$$

Daí, o conjunto $\Psi$ munido da topologia induzida por $d$ é um espaço métrico compacto que chamaremos de realização geométrica de $K$ e denotaremos por $|K|$. Observamos que existe uma cópia de $|K|$ em $\mathbb{R}^{n}$ tal que $\left\{v_{2}-v_{1}, \ldots, v_{n}-v_{1}\right\}$ é um conjunto linearmente independente. Dado um p-simplexo $s=\left\{v_{s_{0}}, \ldots, v_{s_{p}}\right\} \in K$ denotaremos por:

$$
|s|=\left\{\sum_{i=1}^{n} r_{i} v_{i} \in|K| ; r_{i} \neq 0 \Leftrightarrow i \in\left\{s_{0}, \ldots, s_{p}\right\}\right\}
$$

a realização geométrica de $s$. Assim, $|s|$ não contém seu bordo com relação a $|K|$ e cada elemento $\sum_{i=1}^{n} r_{i} v_{i} \in|K|$ está na realização geométrica de um único simplexo de $K$. Dessa forma, dado $x \in|K|$ denotaremos por $\sigma(x)$ o único simplexo de $K$ tal que $x \in|\sigma(x)|$.

Uma subdivisão $K^{\prime}$ de um complexo simplicial $K$ é um complexo simplicial tal que:

1. os vértices de $K^{\prime}$ são pontos de $|K|$ e todo vértice de $K$ é vértice de $K^{\prime}$;

2. se $s^{\prime}$ for um simplexo de $K^{\prime}$, então existe algum simplexo $s \in K$ tal que $\left|s^{\prime}\right| \subset|s|$;

3. a aplicação linear $\left|K^{\prime}\right| \rightarrow|K|$ que leva cada vértice de $K^{\prime}$ em seu correspondente em $|K|$ é um homeomorfismo.

Dado um vértice $\{v\}$ de um complexo simplicial $K$ denotaremos por $S t(v)$ a união de todas as realizações geométricas de simplexos de $K$ que contém $v$, isto é:

$$
S t(v)=\bigcup_{v \in s \in K}|s|
$$


Daí, dado um simplexo $t \in K$ indicaremos por:

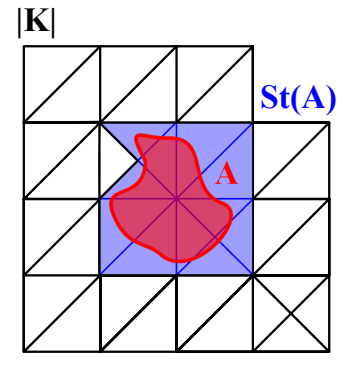

Figura 1.2: $S t(A)$

$$
S t(t)=\bigcup_{\substack{t \cap s \neq \emptyset \\ s \in K}}|s| .
$$

Por fim, dado um subconjunto $A$ de $|K|$ usaremos:

$$
S t(A)=\bigcup_{\substack{|\overline{\mid}| \cap A \neq \emptyset \\ t \in K}}|t| .
$$

Diremos que um espaço topológico $X$ é um poliedro se existirem um complexo simplicial $K$ e um homeomorfismo entre $|K|$ e $X$. Dessa forma, uma triangularização de um poliedro $X$ é um par $(K, \tau)$, onde $K$ é um complexo simplicial e $\tau:|K| \rightarrow X$ é um homeomorfismo. É importante que fique claro que em muitas ocasiões pensaremos em um poliedro $X$ como sendo a realização geométrica de um complexo simplicial $X$.

Sejam um complexo simplicial $K$ e $f: L \rightarrow|K|$ uma aplicação, onde $L$ é um subespaço de $|K|$, diremos que $f$ é uma aplicação proximal em $L$ se para todo $x \in L$ tivermos que $f(x) \in\{y \in$ $|K| ; \quad \sigma(x) \cap \sigma(y) \neq \emptyset\}$, onde $\sigma(x)$ é o único simplexo de $K$ tal que $x \in|\sigma(x)|$. Portanto, toda aplicação proximal $f: L \rightarrow|K|$ possui a propriedade $f(L) \subset S t(L)$.

Agora vamos retomar as notações relacionadas aos caminhos, mas no contexto dos complexos simpliciais. O caminho mais simples que podemos tomar entre dois pontos $a$ e $b$ na mesma componente

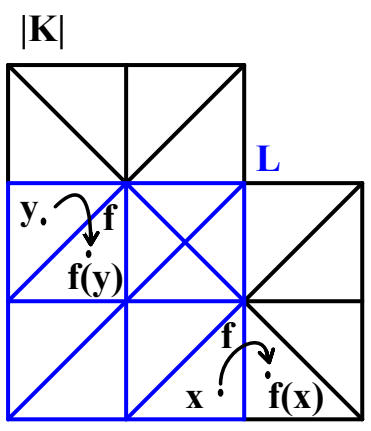

Figura 1.3: Aplicação Proximal conexa da realização geométrica de um simplexo de $|K|$ é o segmento entre $a$ e $b$ dado por $[a, b]=\{x \in|K| ; x=t a+(1-t) b, t \in I\}$. Um caminho $p: I \rightarrow|X|$ na realização geométrica de um complexo simplicial $X$ será chamado de PL-caminho se satisfaz as seguintes condições:

1. $p$ não possui auto-interseção, isto é, não existem $0 \leq t_{0}<t_{1} \leq 1$ tais que $p\left(t_{0}\right)=$ $p\left(t_{1}\right)$

2. existem $0=t_{0}<t_{1}<\cdots<t_{n}=1$ tais que dado $\left.i \in \underline{\{0,1}, \ldots, n-1\right\}$ existe um simplexo $\sigma_{i}$ de $X$ tal que $p\left(\left[t_{i}, t_{i+1}\right]\right)=\left[p\left(t_{i}\right), p\left(t_{i+1}\right)\right] \subset \overline{\left|\sigma_{i}\right|}$;

Além disso, diremos que o PL-caminho $p$ é normal se:

1. os simplexos $\sigma_{i}$ são maximais em $X$ e $p\left(t_{i}\right) \in\left|\sigma_{i-1} \cap \sigma_{i}\right|$, onde $\sigma_{i-1} \cap \sigma_{i}$ é uma face de $\sigma_{i-1}$ e de $\sigma_{i}$ diferente de um vértice, para $i=2, \ldots, n$; 
2. $p(0) \subset\left|\sigma_{0}\right|, p(1) \subset\left|\sigma_{n}\right|$ e $p(] t_{i}, t_{i+1}[) \subset\left|\sigma_{i}\right|$, para $i=0,1, \ldots, n-1$.

Outrossim, dados um PL-caminho normal $q: I \rightarrow X$ e um caminho $p: I \rightarrow X$ diremos que $p$ é especial com relação a $q$ se $p(t) \neq q(t)$ para $t \in] 0,1[, p(0)=q(0)$ e $p(1)=q(1)$, quando não houver risco de ambiguidade diremos apenas que $p$ é especial . Além disso, diremos que dois caminhos $p_{0}: I \rightarrow X$ e $p_{1}: I \rightarrow X$ são especialmente homotópicos se existe homotopia $\left\{p_{t}\right\}_{t \in I}$ conectando $p_{0}$ a $p_{1}$, relativa aos extremos, tal que $p_{t}$ é especial, para todo $t \in I$.

\subsubsection{Fibrados e Fibrações}

Os fibrados e as fibrações serão usados principalmente no terceiro capítulo deste trabalho, mas os apresentamos aqui, apenas no contexto não equivariante, para entendermos as ideias usadas em [19]. Seguiremos principalmente, mas não unicamente, [4, 5, 19].

Um fibrado é uma coleção $\mathfrak{F}=(X, p, B, Y)$ que consiste de uma aplicação $p: X \rightarrow B$, tal que para cada $b \in B$ existe um aberto $U_{b}$ de $B$ que contém $b$ e uma trivialização local consistindo de um homeomorfismos $\phi_{b}: U_{b} \times Y \rightarrow p^{-1}\left(U_{b}\right)$ tais que $p \circ \phi_{b}=\pi$, onde $\pi: U_{b} \times Y \rightarrow U_{b}$ é a projeção na primeira coordenada.

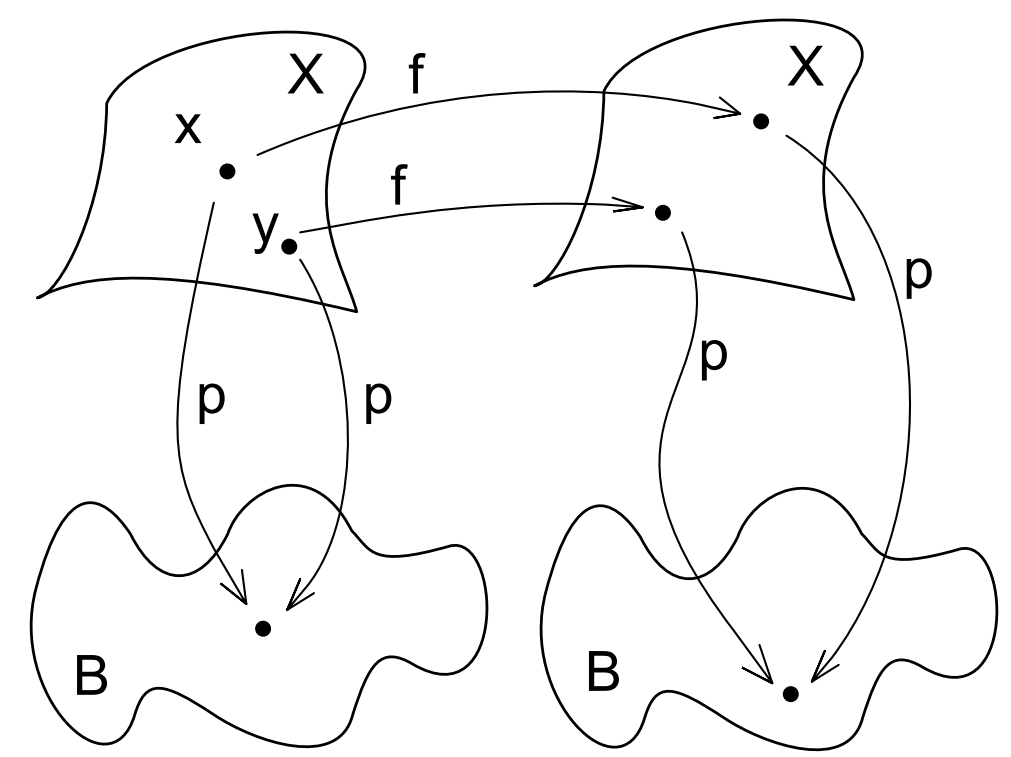

Figura 1.4: Preservar Fibra

Desse modo, dado um fibrado $\mathfrak{F}=(X, p, B, Y)$ e uma aplicação $f: X \rightarrow X$ diremos que $f$ preserva fibra com relação a $\mathfrak{F}$ se dados $x, y \in$ $X$ tais que $p(x)=p(y)$ então $p \circ f(x)=p \circ f(y)$. Assim, $f$ induz uma aplicação $\bar{f}: B \rightarrow B$ tal que $\bar{f} \circ p=p \circ f$.

O conceito de preservar fibra também é usado para homotopias, isto é, dada uma homotopia $H: X \times I \rightarrow X$ diremos que $H$ preserva fibra com relação a $\mathfrak{F}$ se fixado $t \in I$ tivermos que $H(\bullet, t)$ : $X \rightarrow X$ é uma aplicação que preserva fibra. De modo similar ao que ocorre com as aplicações que preservam fibra temos que $H$ induz uma homotopia $\bar{H}: B \times I \rightarrow B$ tal que $\bar{H}(p(x), t)=p \circ H(x, t)$, para todo $(x, t) \in X \times I$.

Para cada fibrado $\mathfrak{F}=(X, p, B, Y)$ existem pelo menos dois possíveis fibrados associados. O primeiro é chamado de par fibrado e é dado por um par topológico $(X, A)$, onde $A$ é um subconjunto fechado de $X$, com a propriedade de existir um subespaço não vazio $Y_{0} \subset Y$ tal que a restrição de cada trivialização local $\phi_{\alpha}: U_{\alpha} \times Y_{0} \rightarrow p^{-1}\left(U_{\alpha}\right) \cap A$ de $\mathfrak{F}$ é um homeomorfismo. Esse novo fibrado é denotado por $\mathfrak{F}^{\prime}=\left(A, p, B, Y_{0}\right)$. 
O segundo é quando tomamos um subconjunto $W$ fechado de $B$ e pensamos em $\left.\mathfrak{F}\right|_{W}=$ $\left(p^{-1}(W), p, W, Y\right)$. Nesse caso, as restrições de cada trivialização local serão $\phi_{\alpha}:\left(U_{\alpha} \cap\right.$ $W) \times Y \rightarrow p^{-1}\left(U_{\alpha} \cap W\right)$. Daí, diremos que um subconjunto $A$ de $X$ é um subconjunto fibrado do fibrado $\mathfrak{F}$ se para cada componente $p(A)_{j}$ de $p(A)$, o par $\left(p^{-1}\left(p(A)_{j}\right), A_{j}\right)$ for um par de fibrados com relação a $\mathfrak{F} \mid p(A)_{j}$, onde $A_{j}=A \cap p^{-1}\left(p(A)_{j}\right)$.

Notemos que quando tomamos o subconjunto fibrado $A$ do fibrado $\mathfrak{F}$ existe a possibilidade de cada componente $p(A)_{j}$ possuir uma fibra $Y_{j} \subset Y$ diferente.

Agora vamos mudar um pouco o foco. Sejam $X$ e $B$ dois espaços relacionados por uma aplicação $p: X \rightarrow B$. Diremos que $p$ possui a propriedade de levantamento de homotopia para um espaço $Z$ quando dadas uma aplicação $f: Z \rightarrow X$ e uma homotopia $F: Z \times I \rightarrow B$ tais que $p \circ f(x)=F(x, 0)$, para todo $x \in Z$, existir uma homotopia $\bar{F}: Z \times I \rightarrow X$ tal que $p \circ \bar{F}=F$ e $\bar{F}(x, 0)=f(x)$, para todo $x \in Z$.

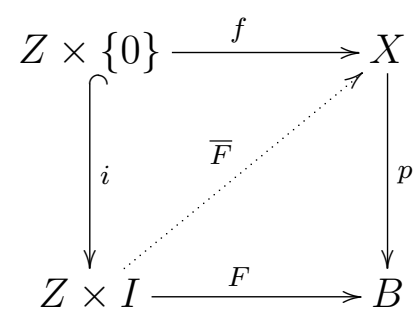

Daí, $p$ é uma fibração para uma classe de espaços $\mathfrak{A}$ se tiver a propriedade de levantamento de homotopias para todo elemento de $\mathfrak{A}$. Quando $\mathfrak{A}$ for a classe de todos os espaços chamaremos $p$ de fibração apenas. Uma fibração é regular se

$$
\{x \in Z ; F(x, t)=F(x, 0), \forall t \in I\} \subset\{x \in Z ; \bar{F}(x, t)=\bar{F}(x, 0), \forall t \in I\} .
$$

Um exemplo de fibração é a fibração trivial onde consideramos quaisquer dois espaços $X$ e $B$ e tomamos a projeção na primeira coordenada $p: B \times X \rightarrow B$. Um exemplo de fibração que não é fibrado é dado tomando $X=\left\{(x, y) \in \mathbb{R}^{2} ; x^{2}+(y-2)^{2} \leq 1\right\}$, $B=\left\{(x, 0) \in \mathbb{R}^{2} ;-1 \leq x \leq 1\right\}$ e $p: X \rightarrow B$ dada por $p(x, y)=$ $(x, 0)$.
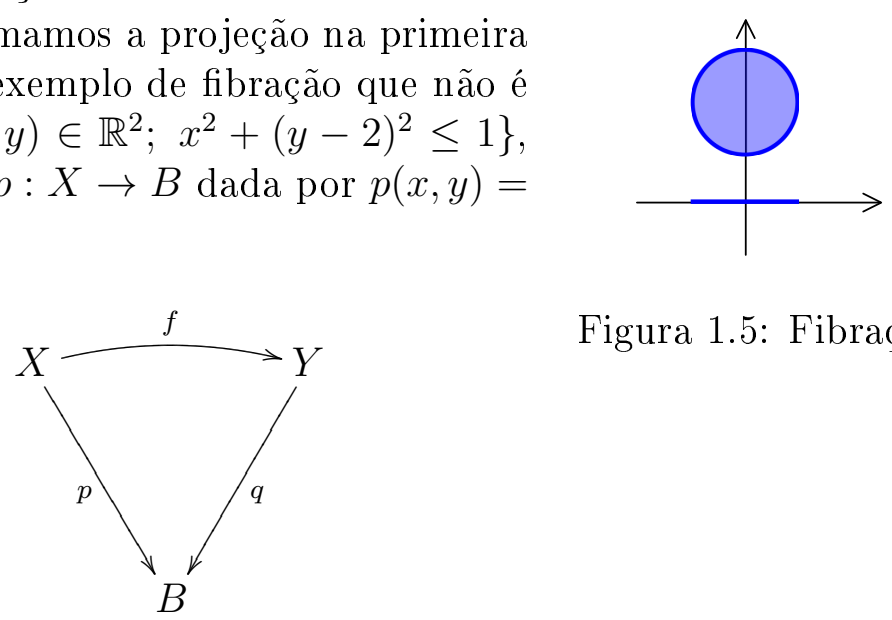

Figura 1.5: Fibração

Dadas duas fibrações $(X, p, B)$ e $(Y, q, B)$ e uma aplicação $f: X \rightarrow Y$, diremos que $f$ é uma aplicação entre fibrações que preserva fibra quando $q \circ f=p$. 


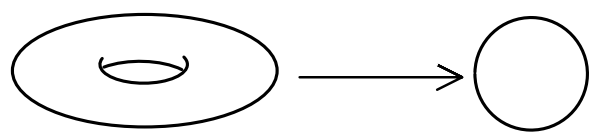

Figura 1.6: Preservar Fibra

Observamos que uma aplicação que preserva fibra com relação a um fibrado pode não ser uma aplicação entre fibrações que preserva fibra. Por exemplo, tomando $\pi_{1}: \mathbb{S}^{1} \times \mathbb{S}^{1} \rightarrow \mathbb{S}^{1}$ a projeção na primeira coordenada temos que $\left(\mathbb{S}^{1} \times \mathbb{S}^{1}, \pi_{1}, \mathbb{S}^{1}\right)$ é uma fibração e $\mathfrak{A}=\left(\mathbb{S}^{1} \times \mathbb{S}^{1}, \pi_{1}, \mathbb{S}^{1}, \mathbb{S}^{1}\right)$ é um fibrado. Daí, $f: \mathbb{S}^{1} \times \mathbb{S}^{1} \rightarrow \mathbb{S}^{1} \times \mathbb{S}^{1}$ dada por $f(\cos (\theta)+i \operatorname{sen}(\theta), z)=(\cos (2 \theta)+i \operatorname{sen}(2 \theta), z)$ é uma aplicação que preserva fibra com relação a $\mathfrak{A}$, pois se $p(a, b)=p(x, y)$ então $a=x$ e $p \circ f(a, b)=p \circ f(x, y)$. Contudo, $f$ não é uma aplicação entre fibrações que preserva fibra, pois nem sempre teremos que $p(\cos (\theta)+i \operatorname{sen}(\theta), z)=\cos (\theta)+i \operatorname{sen}(\theta)$ é igual a

$$
\begin{aligned}
p \circ f(\cos (\theta)+i \operatorname{sen}(\theta), z) & =(\cos (2 \theta)+i \operatorname{sen}(2 \theta), z) \\
& =p(\cos (2 \theta)+i \operatorname{sen}(2 \theta), z) \\
& =\cos (2 \theta)+i \operatorname{sen}(2 \theta) .
\end{aligned}
$$

Duas fibrações $(X, p, B)$ e $(Y, q, B)$ são equivalentes se existirem duas aplicações que preservam fibra $f: X \rightarrow Y$ e $g: Y \rightarrow X$ tais que $f \circ g$ é homotópica a $I d_{Y}$ (vamos denotar essa homotopia por $\phi_{1}$ ) e $g \circ f$ é homotópica a $I d_{X}$ (vamos denotar essa homotopia por $\left.\phi_{2}\right)$ de modo que o caminho $\phi_{i}(e, \bullet)$ está em uma única fibra, para todo $e \in X$ quando $i=2$ ou para todo $e \in Y$ quando $i=1$.

Dados uma fibração $(X, p, B)$, um espaço $Z$ e duas homotopias $\bar{F}: Z \times I \rightarrow X$ e $F: Z \times I \rightarrow B$ tais que $p \circ \bar{F}=F$ diremos que $\bar{F}$ é estacionária com relação a $F$ se para todo $x \in Z$ tal que o caminho $F(x, \bullet): I \rightarrow B$ é constante tivermos que o caminho $\bar{F}(x, \bullet): I \rightarrow X$ também é constante. Daí, diremos que a fibração $(X, p, B)$ é regular se dados um espaço $Z$ e uma homotopia $F: Z \times I \rightarrow B$ pudermos tomar $\bar{F}: Z \times I \rightarrow X$ estacionária com relação a $F$.

Por fim, dados $X$ e $B$ espaços topológicos relacionados por uma aplicação $p: X \rightarrow B$, definimos uma aplicação de levantamento como sendo uma aplicação $\Lambda: \Omega_{p} \rightarrow X^{I}$ tal que $\Lambda(e, \alpha)(0)=e$ e $p \circ \Lambda(e, \alpha)(t)=\alpha(t)$, onde $\Omega_{p}=\left\{(e, \gamma) \in X \times B^{I} ; p(e)=\gamma(0)\right\}$. Quando $\Lambda(e, p(e))=e$, onde $e \in X^{I}$ e $p(e) \in B^{I}$ estão sendo entendidos como os caminhos constantes, então chamaremos $\Lambda$ de aplicação de levantamento regular.

\subsubsection{Classes de Nielsen e Índice de Pontos Fixos}

As notações que apresentaremos nessa subseção estão relacionadas a pontos fixos e foram retiradas principalmente de $[1,7]$.

Dados um espaço topológico $X$ e uma aplicação $f: X \rightarrow X$ diremos que $f$ é compactamente fixada quando o conjunto de pontos fixos de $f$, Fix $(f)=\{x \in X \mid f(x)=$ $x$ \}, for compacto em $X$. Dado $A$ um subespaço de um espaço topológico $X$, diremos que uma homotopia $\left\{h_{t}\right\}_{t \in I}: A \rightarrow X$ é especial se Fix $\left(h_{t}\right)=F i x\left(h_{0}\right)$, para $t \in I$.

Agora vamos apresentar um pouco sobre a teoria de índice de pontos fixos. Sejam $\mathbb{S}^{n}$ a esfera n-dimensional, um subconjunto aberto $V$ de $\mathbb{S}^{n}$, uma aplicação $f: V \rightarrow \mathbb{S}^{n}$ e 
um ponto $Q \in \mathbb{S}^{n}$ tais que $f^{-1}(Q)$ é compacto. Consideremos as seguintes aplicações em homologia sobre os inteiros:

$$
H_{n}\left(\mathbb{S}^{n}\right) \stackrel{i_{*}}{\rightarrow} H_{n}\left(\mathbb{S}^{n}, \mathbb{S}^{n}-f^{-1}(Q)\right) \stackrel{\operatorname{exc}}{\cong} H_{n}\left(V, V-f^{-1}(Q)\right) \stackrel{f_{*}}{\rightarrow} H_{n}\left(\mathbb{S}^{n}, \mathbb{S}^{n}-Q\right) \cong H_{n}\left(\mathbb{S}^{n}\right)
$$

a aplicação exc é o isomorfismo dado pela excisão.

Essa composição é tal que um gerador $o$ de $H_{n}\left(\mathbb{S}^{n}\right)$ é levado na classe $m . o$ onde $m$ é um número inteiro. Nesse caso, diremos que $m$ é o grau local de $f$ sobre $Q$. Uma propriedade importante do grau é que se $f^{-1}(Q) \subset K \subset U \subset V$, onde $K$ é um subconjunto compacto e $U$ uma vizinhança aberta de $K$, então o grau local de $f$ sobre $Q$ também é dado pela composição:

$$
H_{n}\left(\mathbb{S}^{n}\right) \rightarrow H_{n}\left(\mathbb{S}^{n}, \mathbb{S}^{n}-K\right) \stackrel{e x c}{\cong} H_{n}(U, U-K) \stackrel{f_{*}}{\rightarrow} H_{n}\left(\mathbb{S}^{n}, \mathbb{S}^{n}-Q\right) \cong H_{n}\left(\mathbb{S}^{n}\right)
$$

Daí, dado um par $K \subset U$, onde $f^{-1}(Q) \subset K, K$ é compacto e $U$ é uma vizinhança aberta de $K$, diremos que o elemento $o_{K} \in H_{n}(U, U-K)$ que é a imagem de um gerador $o$ de $H_{n}\left(\mathbb{S}^{n}\right)$ é uma classe fundamental ao redor de $K$.

Agora, sejam $V$ um aberto de $\mathbb{R}^{n}, i: V \rightarrow \mathbb{R}^{n}$ a inclusão e uma aplicação $f: V \rightarrow \mathbb{R}^{n}$ compactamente fixada, denotemos $F i x(f)$ por $F$. Então, consideremos a aplicação:

$$
(i-f)_{*}: H_{n}(V, V-F) \rightarrow H_{n}\left(\mathbb{R}^{n}, \mathbb{R}^{n}-\{0\}\right) \cong \mathbb{Z} .
$$

Dessa forma, o índice de pontos fixos $I_{f} \in \mathbb{Z}$ de $f$ é o inteiro tal que $(i-f)_{*}\left(o_{F}\right)=$ $I_{f} \cdot O_{\{0\}}$. Observamos que esta definição não depende da escolha do gerador $o$ de $H_{n}\left(\mathbb{S}^{n}\right)$, pois $(-O)_{F}=-\left(o_{F}\right)$ e $(-o)_{\{0\}}=-\left(o_{\{0\}}\right)$.

Como consequência da propriedade de grau que demos acima temos que se $F i x(f) \subset$ $K \subset U \subset V$, onde $K$ é um subconjunto compacto e $U$ uma vizinhança aberta de $K$, então $i-f$ leva $(U, U-K)$ em $\left(\mathbb{R}^{n}, \mathbb{R}^{n}-\{0\}\right)$ e $(i-f)_{*}\left(o_{K}\right)=I_{f} \cdot o_{\{0\}}$.

Uma generalização do índice que apresentamos é dada quando tomamos $X$ um espaço topológico e um aberto $U$ de $X$ que é um ENR então qualquer aplicação $f: U \rightarrow X$ admite uma decomposição $f=\beta \circ \alpha$ dada por:

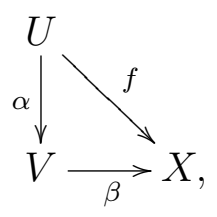

onde $V$ é um aberto de $\mathbb{R}^{n}$ para algum $n$. Podemos construir uma das possíveis $\beta$ e $\alpha$ da seguinte forma: $U$ é um ENR então existe uma vizinhança $V$ em algum espaço euclidiano $\mathbb{R}^{n}$, para algum $n$, de modo que $i: U \hookrightarrow V$ é um homeomorfismo sobre $i(U)$, $r: V \rightarrow i(U)$ é uma retração e $r \circ i(u)=i(u)$ para todo $u \in U$. Assim, existe um homeomorfismo $j: i(U) \rightarrow U$ tal que $j \circ i$ é a identidade $I d_{U}$ de $U$. Daí, tomamos $\alpha=i$ e $\beta=f \circ j \circ r \mathrm{e}$

$$
\beta \circ \alpha=f \circ j \circ r \circ i=f \circ j \circ i=f .
$$


O diagrama que ilustra essa situação é dado abaixo:

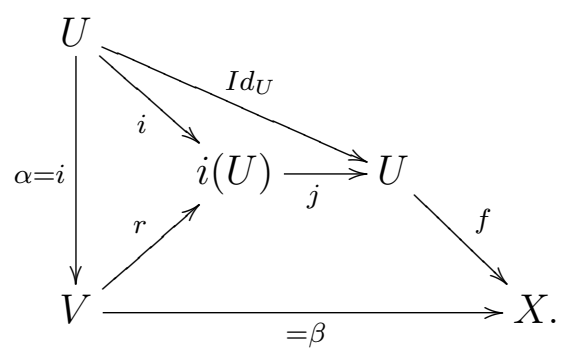

Desse modo, se $f$ for compactamente fixada então podemos usar a aplicação $\alpha \circ \beta$ : $\beta^{-1}(U) \rightarrow V \subset \mathbb{R}^{n}$ para definir $I_{\alpha \circ \beta}$ o índice de pontos fixos de $\alpha \circ \beta$.

A propriedade que torna essa construção importante é que dada qualquer decomposição $f=b \circ a$ temos que $I_{\alpha \circ \beta}=I_{a \circ b}$, isto é, $I_{\alpha \circ \beta}$ depende apenas de $f$. Por isso diremos que $I_{\alpha \circ \beta}=I_{f}$ é o índice de pontos fixos de $f$.

O índice definido para os espaços ENR possuem as seguinte propriedades:

1. se $W$ for um aberto de $X$ tal que $F i x(f) \subset W \subset U$ então $I_{f}=I_{\left.f\right|_{W}}$;

2. dado $x_{0} \in X$ suponhamos que $f$ é a aplicação constante dada por $f(x)=x_{0}$, para todo $x \in U$, então:

$$
I_{f}= \begin{cases}1 & \text { se } x_{0} \in U \\ 0 & \text { se } x_{0} \notin U\end{cases}
$$

3. se $U=\bigcup_{i=1}^{r} U_{i}$, onde $U_{i}$ é aberto para $i=1, \ldots, r$, e $U_{i} \cap U_{j} \cap F i x(f)=\emptyset$ para todo $i \neq j$ então $I_{f}=\sum_{i=1}^{r}\left(I_{\left.f\right|_{U_{i}}}\right)$;

4. se $H: U \times I \rightarrow X$ é uma homotopia e $\bigcup_{t \in I} F i x(H(\bullet, t))$ é compacto então $I_{H(\bullet, t)}=$ $I_{H(\bullet, 0)}$ para todo $t \in I$

5. se $Y$ é um espaço topológico, $W$ é um aberto de $Y$ que é um ENR e $h: W \rightarrow Y$ é uma aplicação compactamente fixada então $I_{f \times h}=I_{f} \cdot I_{h}$, onde $f \times h: U \times W \rightarrow X \times Y$ é a aplicação compactamente fixada dada por $(f \times h)(x, y)=(f(x), h(y))$ para todo $(x, y) \in U \times W$

6. se $Y$ é um espaço topológico, $W$ é um aberto de $Y$ que é um ENR e $h: W \rightarrow X$ e $k: U \rightarrow Y$ são aplicação então o conjunto de pontos fixos de $h \circ k: k^{-1}(W) \rightarrow X$ é homeomorfo ao conjunto de pontos fixos de $k \circ h: h^{-1}(U) \rightarrow Y$. Se esses conjuntos forem compactos então $I_{h \circ k}=I_{k \circ h}$.

Agora vamos particionar o conjunto de pontos fixos de uma aplicação compactamente fixada. Sejam $X$ um espaço topológico ENR conexo e compacto, $f: X \rightarrow X$ uma aplicação e $x, y \in F i x(f) \neq \emptyset(F i x(f)$ é compacto porque é fechado dentro de um compacto), diremos que $x$ e $y$ estão na mesma classe de pontos fixos se, e somente 
se, existir um caminho $\alpha: I \rightarrow X$ tal que $\alpha(0)=x, \alpha(1)=y$ e $\alpha$ é homotópico a $f \circ \alpha$ relativo aos extremos.

Como cada classe de pontos fixos $N$ de $f$ é aberta e fechada em Fix $(f)$ (veja a demonstração do Teorema VI.B.1 de [1] pg 86-87), podemos escolher um aberto $U$ de $X$ tal que $N \subset U$ e $U \cap N^{\prime}=\emptyset$ para toda classe de pontos fixos $N^{\prime} \neq N$ e $I_{\left.f\right|_{U}}$ está bem definido. Daí, diremos que $I(f, N)=I_{\left.f\right|_{U}}$ é o índice da classe de pontos fixos $N$. Observamos que $I(f, N)$ está bem definido porque se for possível tomar um aberto $W$ de $X$ tal que $N \subset W \subset U$ então segue da primeira propriedade do índice para espaços $E N R$ que $I_{\left.f\right|_{U}}=I_{\left.f\right|_{W}}$. Se $I(f, N) \neq 0$ então $N$ é chamada uma classe de pontos fixos essencial de $f$. O número de Nielsen de $f$, que denotamos por $\mathcal{N}(f)$, é a quantidade de classes de pontos fixos essenciais que $f$ possui.

\subsubsection{Realização de um Conjunto como Conjunto de Pontos Fixos de uma dada Aplicação}

Nessa subseção, que encerra o primeiro compêndio, faremos um esboço das demonstrações dos resultados que generalizaremos para o contexto equivariante. Antes de começar, é importante saber que dados um espaço topológico $X$ e uma aplicação $f: X \rightarrow X$, denotaremos por Fix $(f)=\{x \in X \mid f(x)=x\}$ o conjunto de pontos fixos de $f$. O objetivo do capítulo 2 será generalizar o Lema 3.1 de [9] (pg 157) que é o primeiro resultado que apresentamos.

Lema 1.1 Seja $f: X \rightarrow X$ uma auto-aplicação de um poliedro conexo, compacto $X$ sem pontos de corte local e $A$ um subpoliedro. Suponha que $X-A$ não é uma dois variedade e que A é contornável em $X$. Se (C1) e (C2) valerem então existe uma aplicação $h: X \rightarrow X$ homotópica a f com Fix $(h)=A$.

(C1) Existe uma homotopia $\bar{H}: A \times I \rightarrow X$ entre $\bar{H}(\bullet, 0)=\left.f\right|_{A}$ e $\bar{H}(\bullet, 1)=i: A \hookrightarrow X$ (inclusão).

(C2) Para toda classe de pontos fixos essencial $F$ de $f$ existe um caminho $p: I \rightarrow X$ tal que $p(0) \in F, p(1) \in A$ e $\{p(t)\}$ é homotópica à $\bar{H}(p(t), t)$ (com pontos extremos fixados).

Ideia da Prova: Primeiramente, estendemos a homotopia $\bar{H}$ dada em $(C 1)$ para uma homotopia $H_{1}: X \times I \rightarrow X$, o que é cabível pela propriedade de extensão de homotopias. Em seguida, concatenamos uma segunda homotopia $H_{2}: X \times I \rightarrow X$ relativa a $A$ que nos leva a uma aplicação $h_{2}$ com a propriedade de possuir uma quantidade finita de pontos fixos em $X-A$. Como $H_{2}: X \times I \rightarrow X$ é relativa a $A$ temos que $A \subset F i x\left(h_{2}\right)$.

Daí, unimos os pontos fixos que são Nielsen relacionados e que estão fora de $A$. Depois, enviamos os pontos fixos que estão fora de $A$ e que são Nielsen relacionados aos pontos de $A$ para $A$ e eliminamos, de modo usual, as classes de pontos inessenciais que restaram fora de $A$. 
Seja $H$ a homotopia construída entre $f$ e a aplicação $h$, então $F i x(h)=A$. De fato, se existir $x \in F i x(h)-A$ então $\{x\}$ é uma classe de pontos fixos essencial de $h$ que não está relacionada com nenhuma classe de pontos fixos em $A$. Contudo, $\{x\}$ está $H$-relacionada com uma classe de pontos fixos essencial $F$ de $f$. Por $(C 2)$, existe um caminho $p: I \rightarrow X$ tal que $p(0) \in F, p(1) \in A$ e $\{p(t)\}$ é homotópica à $H(p(t), t)$ (com pontos extremos fixados). Mas,

$$
H(p(1), 1)=h(\underbrace{p(1)}_{\in A})=\underbrace{p(1)}_{\in\{x\}}=x,
$$

o que é um absurdo. Logo, Fix $(h)=A$.

O segundo resultado que trataremos é o Teorema 4.1 de [19] (pg 49) que será generalizado no capítulo 3.

Teorema 1.2 Seja $\mathfrak{F}=(E, p, B, Y)$ um fibrado onde $E, B$ e $Y$ são poliedros conexos $e$ $B$ e $Y$ não possuem pontos de corte local.

Seja $A$ um subconjunto fechado e localmente contrátil de $E$ tal que $(E, A)$ é um par de fibrados para $\mathfrak{F}, p(A)$ é um subconjunto fechado e localmente contrátil de $B$, cada componente $p(A)_{j}$ de $p(A)$ é contrátil em $B, p(A)$ é contornável em $B$ e $B-p(A)$ não é uma 2-variedade.

Seja $f: E \rightarrow E$ uma aplicação que preserva fibra tal que:

$(C 1)_{\mathfrak{F}}$ existe uma homotopia parcial que preserva fibra $H_{A}:(E \times\{0\}) \cup(A \times I) \rightarrow E$ tal que $H_{A}(\bullet, 0)=f$ e $H_{A}(\bullet, 1)=i: A \hookrightarrow E($ inclusão $)$;

$(C 2)_{\mathfrak{F}}$ toda classe de pontos fixos essencial $F$ de $f$ está conectada a A por um caminho $\gamma: I \rightarrow E$ tal que $\gamma(0) \in F, \gamma(1) \in A$ e $\{\gamma(t)\}$ é homotópica à $\{f \circ \gamma\} *\left\{H_{A}(\gamma(1), t)\right\}$ com pontos extremos fixados.

Supondo ainda que para todo subfibrado $Y_{j}$ de A temos que $Y_{j}$ é um subespaço fechado, localmente contrátil e contornável em $Y$ tal que $Y-Y_{j}$ não é uma 2-variedade e que $A$ intercepta todas as classes de pontos fixos essenciais de $f_{b_{j}}: p^{-1}\left(b_{j}\right) \rightarrow p^{-1}\left(b_{j}\right)$ para pelo menos um $b_{j}$ na componente $p(A)_{j}$. Se $Z$ é um subconjunto fechado e fibrado de $A$ que intercepta todas as componentes de $A$, então existe uma aplicação $g: E \rightarrow E$ que é homotópica a $f$ por uma homotopia que preserva fibra tal que Fix $(g)=Z$.

Ideia da Prova: Primeiramente provamos que $f$ é homotópica a uma aplicação $h$ tal que:

$$
A \subset \operatorname{Fix}(h) \subset p^{-1}(p(A)),
$$

e que $\bar{h}: B \rightarrow B$ possui uma quantidade finita de pontos fixos em $B-p(A)$, nenhum desses pontos fixos está na imagem por $p$ de uma classe de pontos fixos essencial de $h$.

Para tanto, tomamos a extensão $\bar{H}_{1}: B \times I \rightarrow B$ da aplicação $\bar{H}_{A}: p(A) \times I \rightarrow B$ induzida por $\bar{H}_{A}$ dada em $(C 1)_{\mathfrak{F}}$ e eliminamos a maior quantidade possível de pontos 
fixos da aplicação $\bar{H}_{1}(\bullet, 1)$ que estão em $p(A)$. Observamos que, nessa passagem usamos quase todas as hipóteses que temos relacionadas com $p(A)$ e que os pontos fixos residuais de $\bar{H}(\bullet, 1)=\bar{h}$ em $B-p(A)$ não podem ser eliminados porque $(C 2)_{\mathfrak{F}}$ é uma hipótese em $E$ que não temos como levar para $B$, onde $\bar{H}$ é a homotopia resultante de todos esses procedimentos em $B$.

Daí, levantando $\bar{H}$ criamos uma homotopia que preserva fibra com relação a $\mathfrak{F} H_{1}$ : $X \times I \rightarrow X$ tal que $f(x)=H_{1}(x, 0)$ e $h_{1}(x)=H_{1}(x, 1)$, para todo $x \in X . h_{1}=H_{1}(\bullet, 1)$ não possui classes de pontos fixos essenciais na imagem inversa dos pontos fixos residuais de $\bar{h}$, com isso criamos $H_{2}: X \times I \rightarrow X, h_{2}=H_{2}(\bullet, 1)$, tal que Fix $\left(h_{2}\right) \subset p^{-1}(p(A))$. Para achar $h: X \rightarrow X$ homotópica a $f$ tal que $A \subset F i x(h) \subset p^{-1}(p(A))$ basta perceber que $C 1_{\mathfrak{F}}$ dá condição para $h_{2}$ ser homotópica a inclusão $i_{A}$ por uma homotopia parcial. Denotaremos essa homotopia parcial por $\widetilde{H}_{A},\left.\operatorname{com} h_{2}\right|_{A}=\widetilde{H}_{A}(\bullet, 0)$ e $i_{A}=\widetilde{H}_{A}(\bullet, 1)$. Daí, definimos:

$$
\begin{aligned}
\widetilde{H}:(X \times\{0\}) \cup\left(p^{-1}\left(\left(\left\{b_{1}, \ldots, b_{r}\right\}\right) \cup p(A)\right) \times I\right) & \rightarrow X \\
(x, t) & \mapsto \begin{cases}h_{2}(x), & \text { se } t=0 ; \\
h_{2, b_{j}}(x), & \text { se } x \in p^{-1}\left(b_{j}\right) ; \\
\widetilde{H}_{A}(x, t), & \text { se } x \in A,\end{cases}
\end{aligned}
$$

onde $b_{i}$ são os pontos fixos residuais de $\bar{h}$. Estendemos agora $\widetilde{H}$ para uma homotopia que preserva fibra $H: X \times I \rightarrow X$.

Na segunda parte da prova analisamos a relação de $h \operatorname{com} p^{-1}\left(p(A)_{j}\right)$. Tomamos $h_{j}$ : $p^{-1}\left(p(A)_{j}\right) \rightarrow p^{-1}\left(p(A)_{j}\right)$ uma restrição de $h$. Daí, provamos que existe uma homotopia que preserva fibra

$$
H_{j}: p^{-1}\left(p(A)_{j}\right) \times I \rightarrow p^{-1}\left(p(A)_{j}\right),
$$

tal que $H_{j}(e, 0)=h_{j}(e)$ para todo $e \in p^{-1}\left(p(A)_{j}\right)$ e $F i x\left(H_{j}(\bullet, 1)\right)=Z \cap A_{j}$.

Isso é feito observando que $\left(p^{-1}\left(p(A)_{j}\right), p, p(A)_{j}\right)$ é uma fibração equivalente a fibração trivial $p(A)_{j} \times Y_{j}$ e que existe homeomorfismo $\Phi: p(A)_{j} \times Y_{j} \rightarrow p^{-1}\left(p(A)_{j}\right)$. Assim, através de uma série de construções se estabelece uma relação biunívoca entre as classes de pontos fixos de $h_{j}$ e $h_{b_{0}}^{*}$, onde $h^{*}=\Phi^{-1} \circ h_{j} \circ \Phi: p(A)_{j} \times Y_{j} \rightarrow p(A)_{j} \times Y_{j}$ é dada por $h^{*}(b, y)=\left(b, h_{b}^{*}(y)\right)$ e $b_{0}$ é um ponto no qual $p(A)_{j}$ se contrai.

Nesse ponto usamos quase todas as hipóteses relacionadas ao subfibrado $Y_{j}$ para provar que $h_{b_{0}}^{*}$ é homotópica a uma aplicação $g_{b_{0}}^{*}: Y_{j} \rightarrow Y_{j}$ tal que Fix $\left(g_{b_{0}}^{*}\right)=\Omega$, onde o par fibrado $\left(\left(p^{-1}\left(p(A)_{j}\right) \cap A, p^{-1}\left(p(A)_{j}\right) \cap Z\right), p, p(A)_{j},\left(Y_{j}, \Omega\right)\right)$ é tal que o conjunto $\Omega$ intercepta todas as componentes conexas de $Y_{j}$. Definimos a homotopia $H^{*}$ entre $h^{*} \mathrm{e}$ $i d \times g_{b_{0}}^{*}$. Lembrando que, Fix $\left(i d \times g_{b_{0}}^{*}\right)=p(A)_{j} \times \Omega$.

Daí, definimos a homotopia $H_{j}: p^{-1}\left(p(A)_{j}\right) \times I \rightarrow p^{-1}\left(p(A)_{j}\right)$ dada por $H(e, t)=$ $\Phi \circ H^{*}\left(\Phi^{-1}(e), t\right)$. Assim, $H(e, 0)=h_{j}(e)$ e $\operatorname{Fix}(H(\bullet, 1))=\Phi\left(p(A)_{j} \times \Omega\right)=p^{-1}\left(p(A)_{j}\right) \cap Z$.

Por fim, seja $F=F i x(h) \cap(B-p(A))$, definimos a seguinte homotopia:

$$
\begin{aligned}
H:[E \times\{0\})] \cup\left[\left(p^{-1}(F) \cup p^{-1}(p(A))\right) \times I\right] & \rightarrow E \\
(e, t) & \mapsto \begin{cases}f(e) & \text { se } t=0 \text { ou } p(e) \in F \\
H_{j}(e, t) & \text { se } p(e) \in p(A)_{j}\end{cases}
\end{aligned}
$$


Essa homotopia parcial pode ser estendida para uma homotopia que preserva fibra $G: E \times I \rightarrow E$ tal que $p \circ G(e, t)=\bar{h} \circ p(e)$ para todo $e \in E$ e todo $t \in I$. Logo, a aplicação $G(\bullet, 1)=g$ preserva fibra, é homotópica a $f$ e $F i x(g)=Z$. 


\subsection{Definições Relativas aos Grupos de Transformações}

Nessa segunda seção apresentaremos um pouco da teoria dos Grupos de Transformações e uma ponte entre tal teoria e os objetos apresentados na seção anterior. Também faremos aqui algumas observações relacionadas à teoria de Nielsen para aplicações equivariantes.

\subsection{1 $G$-espaços e $G$-aplicações}

Uma ação de um grupo $G$ em um conjunto $X$ é uma função $\varphi: G \times X \rightarrow X$ tal que:

1. $\varphi(1, x)=x$ para todo $x \in X$ e $1 \in G$ é o elemento identidade de $G$;

2. $\varphi\left(g_{2}, \varphi\left(g_{1}, x\right)\right)=\varphi\left(g_{2} . g_{1}, x\right)$ para todos $g_{1}, g_{2} \in G$ e $x \in X$.

Quando existir uma ação de um grupo $G$ em um conjunto $X$ diremos que $X$ é um $G$ conjunto e que $\varphi$ é uma $G$-ação de $G$ em $X$. O grupo $G$ é chamado de grupo topológico se $G$ for um espaço topológico Hausdorff e a função:

$$
\begin{aligned}
\alpha: G \times G & \rightarrow G \\
(g, h) & \mapsto g \cdot h^{-1},
\end{aligned}
$$

for contínua. Daí, dados um grupo topológico $G$ e um espaço topológico $X$ diremos que $X$ é um $G$-espaço se existir uma ação contínua $\varphi: G \times X \rightarrow X$.

Um subconjunto $A$ de um $G$-espaço $X$ é $G$-invariante, ou apenas invariante quando não houver risco de ambiguidade, quando $g A \subset A$ para todo $g \in G$. Dada uma aplicação $f: X \rightarrow Y$ entre os $G$-espaços $X$ e $Y$ diremos que $f$ é uma $G$-aplicação, ou que $f$ é equivariante, quando $f(g x)=g(f(x))$ para todo $g \in G$. Uma homotopia $H: X \times I \rightarrow Y$ entre os $G$-espaços $X$ e $Y$ é uma $G$-homotopia quando $H(\bullet, t): X \rightarrow Y$ for uma $G$ aplicação para cada $t \in I$.

Quando existir um $G$-subespaço $A$ de $X$ tal que $H(x, t)=x$, para todo $(x, t) \in A \times I$, diremos que a $G$-homotopia $H$ é relativa a $A$. Observamos que no caso de $G$-homotopias a ação de $G$ em $I$ é trivial.

Consideremos espaços topológicos $X$ e $B$, uma aplicação sobrejetora $p: X \rightarrow B$ e o conjunto $\Omega_{p}=\left\{(e, \gamma) \in X \times B^{I} ; p(e)=\gamma(0)\right\}$, onde $B^{I}$ é o espaço dos caminhos em $B$. Notemos que se $G$ for um grupo finito, $X$ e $B$ forem $G$-espaços e $p$ for equivariante então podemos definir $G$-ações em $X^{I}$ e em $\Omega_{p}$ dadas por:

$$
\begin{array}{rlrlll}
G \times X^{I} & \rightarrow X^{I} & & & G \times \Omega_{p} & \rightarrow \Omega_{p} \\
(g, \alpha) & \mapsto(g \alpha): & I & \rightarrow X & (g,(e, \gamma)) & \mapsto(g e, g \gamma) . \\
& & t & \mapsto(g \alpha)(t)=g(\alpha(t)) & &
\end{array}
$$

A ação em $\Omega_{p}$ está bem definida porque $p(g e)=g p(e)=g \gamma(0)$.

Dado um subgrupo fechado $K$ do grupo topológico $G$ usaremos as seguintes notações:

1. $N K=\left\{g \in G ; g h g^{-1} \in K\right.$ para todo $\left.h \in K\right\}$ o normalizador de $K$ em $G$; 
2. $W K=\frac{N K}{K}$ o grupo de Weyl de $K$ em $G$;

3. $(K)=\left\{g h g^{-1} \in G ; g \in G\right.$ e $\left.h \in K\right\}$ a classe de conjugação de $K$ em $G$. Dadas duas classes $\left(K_{1}\right)$ e $\left(K_{2}\right)$ diremos que $\left(K_{1}\right) \leq\left(K_{2}\right)$ se e somente se existe algum $g \in G$ tal que $g K_{1} g^{-1}$ é um subgrupo de $K_{2}\left(g K_{1} g^{-1} \leq K_{2}\right)$;

4. Dado um ponto $x \in X$, denotamos por $G_{x}=\{g \in G ; g x=x\}$ o subgrupo de isotropia de $x$. $G_{x}$ é um subgrupo de $G$, daí, diremos que $\left(G_{x}\right)$ é um tipo de isotropia de $X$. Denotaremos por $I$ so $(X)$ o conjunto de todos os tipos de isotropias de $X$;

5. $X^{K}=\left\{x \in X ; K \leq G_{x}\right\}$;

6. $X^{(K)}=\left\{x \in X ; g K g^{-1} \leq G_{x}\right.$ para algum $\left.g \in G\right\}$

7. $X_{K}=\left\{x \in X ; G_{x}=K\right\}$;

8. $X_{(K)}=\left\{x \in X ; G_{x} \subset(K)\right\}$

Será importante para a leitura desse trabalho ter todas essas notações em mente, pois serão muito usadas em vários momentos. Quando tomamos $\left\{\left(K_{i}\right)\right\}_{i=1}^{n}$ uma subcoleção finita de subgrupos de $G$ podemos parcialmente ordená-la de forma que se $\left(K_{j}\right) \leq\left(K_{i}\right)$ então $i \leq j$. Uma tal forma de ordenar $\left\{\left(K_{i}\right)\right\}$ é chamada de uma ordem admissível para $\left\{\left(K_{i}\right)\right\}$.

Assim, supondo que $I s o(X)=\left\{\left(K_{i}\right)\right\}_{i=1}^{n}$ é finito e que está munido de uma ordem admissível, definimos a cadeia (ou filtração ) $X_{1} \subset \cdots \subset X_{n}=X$ de $G$-subespaços de $X$ dada por:

$$
X_{i}=\left\{x \in X ;\left(G_{x}\right)=\left(K_{j}\right) \text { para algum } j \leq i\right\} .
$$

Dessa forma, dado $l \in\{2,3, \ldots, n\}$ temos que $X_{l}^{K_{l}}-X_{l-1}^{K_{l}}=X_{K_{l}}$. Observamos que sempre que $G$ for finito teremos que $I s o(X)$ será finito.

Sejam $X$ um $G$-espaço tal que $I s o(X)$ é finito e $X_{1}, \ldots, X_{n}$ a filtração associada a uma ordem admissível de $I s o(X)$. Notemos que toda $G$-aplicação $f: X \rightarrow X$ preserva filtração, isto é, $f\left(X_{i}\right) \subset X_{i}$, para $i=1, \ldots, n$. De fato, dados $x \in X_{i}$ e $g \in G_{x}$ temos que $g f(x)=f(g x)=f(x)$. Daí, $G_{x} \subset G_{f(x)}$ e assim $\left(G_{f(x)}\right)=\left(K_{j}\right)$ com $j \leq i$. Portanto, $f\left(X_{i}\right) \subset X_{i}$.

Ainda com relação a $f$, temos que $W K$ age em $X^{K}$ e que a aplicação $f^{K}: X^{K} \rightarrow X^{K}$ é uma $W K$-aplicação, pois dados $x \in X^{K}, g K \in W K$ e $h \in K$ temos que:

$$
h((g K) x)=\left(g g^{-1} h\right)(g x)=g(\underbrace{g^{-1} h g}_{\in K}) x=g x=(g K) x .
$$

Portanto, $(g K) x \in X^{K}$.

Além disso, $W K_{i}$ age livremente em $X^{K_{i}}-X_{i-1}$. De fato, dado $x \in X^{K_{i}}-X_{i-1}$ temos que $K_{i} \leq G_{x}$ e existe $l \in G$ tal que $l G_{x} l^{-1}=K_{i}$. Dessa forma, $l \in N K_{i}$, 
pois $l K_{i} l^{-1} \leq l G_{x} l^{-1} \leq K_{i}$. Se $g K_{i} \in W K_{i}$ for tal que $x=\left(g K_{i}\right) x=g x$ temos que $g \in G_{x} \cap N K_{i}$. Consequentemente, $g \in l^{-1} K_{i} l \leq K_{i}$ e $g K_{i}=K_{i}$. Logo, $W K_{i}$ age livremente em $X_{i}^{K_{i}}-X_{i-1}^{K_{i}}$.

Até o momento estabelecemos notações para $G$-espaços arbitrários sem estruturas particulares. Contudo, usaremos $G$-espaços com estruturas específicas e por isso precisaremos definir mais conceitos. Sejam $U \subset \mathbb{R}^{n}$ e $V \subset \mathbb{R}^{m}$ dois abertos de seus respectivos espaços. Dada uma aplicação $f: U \rightarrow V, f(x)=\left(f_{1}(x), \ldots, f_{m}(x)\right)$, diremos que a aplicação $f_{i}$ é a i-ésima função coordenada de $f$, para $i=1, \ldots, m$, e diremos que $f$ é suave ou de classe $C^{\infty}$ se cada função coordenada de $f$ possuir derivadas parciais contínuas de todas as ordens.

Dados $X$ um espaço topológico, $U$ um aberto de $X$ e um homeomorfismo $\varphi: U \rightarrow$ $V \subset \mathbb{R}^{n}$ de $U$ em um aberto $V$ de $\mathbb{R}^{n}$ expressaremos por $(U, \varphi)$ a carta de $X$ relacionada $\operatorname{com} \varphi$. Uma coleção $\left\{\left(U_{\alpha}, \varphi_{\alpha}\right)\right\}_{\alpha \in \Lambda}$ de cartas de $X$ tal que a coleção $\left\{U_{\alpha}\right\}_{\alpha \in \Lambda}$ é uma cobertura de $X$ é chamado de atlas de $X$.

Um atlas $\left\{\left(U_{\alpha}, \varphi_{\alpha}\right)\right\}_{\alpha \in \Lambda}$ de $X$ é suave se para cada par de índices $\alpha, \beta \in \Lambda$ tais que $U_{\alpha} \cap U_{\beta} \neq \emptyset$ a aplicação $\varphi_{\beta} \circ \varphi_{\alpha}^{-1}: \varphi_{\alpha}\left(U_{\alpha} \cap U_{\beta}\right) \rightarrow \varphi_{\beta}\left(U_{\alpha} \cap U_{\beta}\right)$ é suave.

Um espaço topológico $X$, Hausdorff e com base enumerável, munido de um atlas suave $\left\{\left(U_{\alpha}, \varphi_{\alpha}\right)\right\}_{\alpha \in \Lambda}$ é chamado de variedade suave ou variedade diferenciável. Diremos que $G$ é um grupo de Lie se $G$ possuir as estruturas de grupo topológico, a de variedade suave e a aplicação $\alpha: G \times G \rightarrow G$, dada por $\alpha(g, h)=g \cdot h^{-1}$, for suave.

Notemos que todo grupo finito é um grupo de Lie, tendo isso em mente, introduziremos algumas definições que vimos nas subseções anteriores no contexto equivariante.

Sejam $G$ um grupo de Lie e $(X, A)$ um par de $G$-espaços, diremos que $A$ é um $G$ retrato de uma vizinhança de $X$ se existir um aberto $G$-invariante $V \subset X$, com $A \subset V$, e existir uma $G$-retração $r: V \rightarrow A$. Nesse caso, diremos que $V$ se retrai equivariantemente em $A$. Além disso, diremos que $A$ é um $G$-retrato forte de uma vizinhança de $X$ quando a $G$-retração $r: V \rightarrow A$ é tal que $i \circ r$ é $G$-homotópica a identidade de $V\left(i \circ r \approx I d_{V}\right)$, relativo a $A$, onde $i: A \hookrightarrow V$ é a inclusão.

Dado $G$ um grupo de Lie, diremos que um $G$-espaço métrico $X$ é um $G$-ANR se, e somente se, existe um $G$-mergulho $i: X \hookrightarrow Y$, onde $Y$ é um $G$-espaço métrico separável e $i(X)$ é um $G$-retrato de uma vizinhança de $Y$.

Dados $G$ um grupo de Lie e $X$ um $G$-espaço métrico, diremos que $X$ é um $G$-ENR se, e somente se, existe um $G$-mergulho $i: X \hookrightarrow E$ de $X$ em um $G$-espaço euclidiano $E$ tal que $i(X)$ é um $G$-retrato de uma vizinhança de $E$. Diremos que $(X, A)$ é um $G$-par topológico quando $X$ for um $G$-espaço (munido da ação contínua $\varphi: G \times X \rightarrow X$ ) e $A$ um subespaço topológico de $X$ tal que $A$ munido da ação $\left.\varphi\right|_{G \times A}$ é um $G$-espaço topológico. Um par $G$-ENR $(X, A)$ é um $G$-par topológico tal que $X$ é um $G$-ENR (munido da ação contínua $\varphi: G \times X \rightarrow X)$ e $A$ um subespaço fechado de $X$ tal que $A$ munido da ação $\left.\varphi\right|_{G \times A}$ é um $G$-ENR.

Vejamos agora a versão equivariante para as definições relativas aos complexos simpliciais. Dados $K$ um complexo simplicial e $G$ um grupo finito que age em $K$, diremos que a ação de $G$ em $K$ é simplicial se para todo $g \in G$ a aplicação $g: K \rightarrow K$ induzida 
pela ação de $G$ em $K$ é uma aplicação simplicial.

Quando a ação de $G$ em $K$ for simplicial diremos que $K$ é um $G$-complexo simplicial. Observamos que existem ações de grupos infinitos em complexos simpliciais, mas esses não usaremos.

A versão equivariante para triangularizações ocorre de forma muito natural quando $G$ for um grupo finito. Nesse caso, diremos que um $G$-espaço $X$ é equivariantemente triangularizável se existir um homeomorfismo equivariante $\tau:|K| \rightarrow X$, onde $K$ é um $G$-complexo simplicial e $|K|$ a realização geométrica do complexo simplicial $K$.

Observamos que quando $G$ é finito e $X$ é uma $G$-variedade suave e compacta (com ou sem bordo) temos que $X$ é equivariantemente triangularizável, ver [23]. Além disso, existirá uma métrica $\rho$ no poliedro $X$ que usamos para definir a métrica $G$-invariante de $X$ dada por:

$$
d(x, y)=\frac{1}{|G|} \sum_{g \in G} \rho(g x, g y) .
$$

\subsection{2 Índice de Pontos Fixos Equivariante}

As notações que apresentaremos nessa subseção estão relacionadas a pontos fixos no contexto equivariante, foram retiradas principalmente de $[13,16]$ a fim de completar as informações dadas na Subseção 1.1.4 para o contexto equivariante.

Definição 1.3 Sejam $G$ um grupo finito, $Y$ um $G$-ENR conexo e compacto, $h: Y \rightarrow Y$ uma $G$-aplicação e $x, y \in F i x(h) \neq \emptyset$. Então $x$ e $y$ estão na mesma $G$-classe de pontos fixos se, e somente se, ocorre uma dentre as seguintes:

(1) $y=\sigma x$ para algum $\sigma \in G$;

(2) existe um caminho $\alpha: I \rightarrow Y$ tal que $\alpha(0)=x, \alpha(1)=\sigma^{\prime} y$ para algum $\sigma^{\prime} \in G$ e $\alpha \approx h \circ \alpha$ relativo aos extremos.

Como cada $G$-classe de pontos fixos $N$ de $h$ é aberta e fechada em Fix $(h)$, podemos proceder como no caso não equivariante e escolher um aberto $G$-invariante $U$ de $X$ tal que $N \subset U$ e $U \cap N^{\prime}=\emptyset$ para toda $G$-classe de pontos fixos $N^{\prime} \neq N$ e $I_{\left.h\right|_{U}}$ está bem definido. Daí, diremos que $I(h, N)=I_{\left.h\right|_{U}}$ é o índice da $G$-classe de pontos fixos $N$. Se $I(h, N) \neq 0$ então $N$ é chamada uma $G$-classe de pontos fixos essencial de $h$ e quando $I(h, N)=0$ então $N$ é chamada uma $G$-classe de pontos fixos não essencial (ou inessencial) de $h$.

Por fim, observamos que uma $G$-classe de pontos fixos $N$ de $h$ é formada pela união de classes de pontos fixos $\left\{N_{i}\right\}_{i \in \Lambda}$ de $h$, ignorando a ação de $G$, e que $I(h, N)=\sum_{i \in \Lambda} I\left(h, N_{i}\right)$.

\subsubsection{Aplicações que Preservam Fibra}

$\mathrm{Na}$ última parte desse segundo compêndio faremos considerações referentes a fibrados e fibrações no contexto equivariante, que serão usados principalmente no capítulo 3. 
Dado um fibrado $\mathfrak{F}=(X, p, B, Y)$ temos que para cada $b \in B$ existe um aberto $U_{b}$ de $B$ que contém $b$ e uma trivialização local $\phi_{b}: U_{b} \times Y \rightarrow p^{-1}\left(U_{b}\right)$ tais que $p \circ \phi_{b}=\pi$. Daí, dado um grupo de Lie compacto $G$, se $X, B$ e $Y$ forem $G$-espaços, os abertos $U_{b}$ de $B$ forem $G$-invariantes e $p$ e $\phi$ forem $G$-aplicações diremos que $\mathfrak{F}$ é um $G$-fibrado.

Assim, dados um $G$-fibrado $\mathfrak{F}=(X, p, B, Y)$ e uma $G$-aplicação $f: X \rightarrow X$ que preserva fibra com relação a $\mathfrak{F}$ temos que $f$ induz uma $G$-aplicação $\bar{f}: B \rightarrow B$ tal que $\bar{f} \circ p=p \circ f$.

Com um $G$-fibrado $\mathfrak{F}=(X, p, B, Y)$ é possível construir dois $G$-fibrados associados de forma análoga a feita no contexto não equivariante. Daí, criamos o $G$-fibrado $\mathfrak{F}^{\prime}=$ $\left(A, p, B, Y_{0}\right)$ chamado $G$-par fibrado e dado por um $G$-par topológico $(X, A)$, onde $A$ é um $G$-subconjunto fechado de $X$, com a propriedade de existir um $G$-subespaço não vazio $Y_{0} \subset Y$ tal que a restrição de cada trivialização local $\phi_{\alpha}: U_{\alpha} \times Y_{0} \rightarrow p^{-1}\left(U_{\alpha}\right) \cap A$ de $\mathfrak{F}$ é um $G$-homeomorfismo.

Criamos também o $G$-fibrado $\left.\mathfrak{F}\right|_{W}=\left(p^{-1}(W), p, W, Y\right)$, onde $W$ é um subconjunto fechado e $G$-invariante de $B$. Além disso, um subconjunto $G$-invariante $A$ de $X$ é um $G$-subconjunto fibrado do $G$-fibrado $\mathfrak{F}$ se para cada componente $p\left(A_{j}\right)$ de $p(A)$, o par $\left(p^{-1}\left(p(A)_{j}\right), A_{j}\right)$ for um $G$-par de fibrados com relação a $\mathfrak{F} \mid p(A)_{j}$, onde $A_{j}=A \cap$ $p^{-1}\left(p(A)_{j}\right)$.

Por outro lado, se $G$ for um grupo finito temos que uma $G$-fibração é uma $G$-aplicação $p: X \rightarrow B$ com a propriedade de levantamento de $G$-homotopias para qualquer $G$-espaço, e $p$ possui a propriedade de levantamento de $G$-homotopia para um $G$-espaço $Z$ quando dadas uma $G$-aplicação $f: Z \rightarrow X$ e uma $G$-homotopia $F: Z \times I \rightarrow B$ tais que $p \circ f(x)=F(x, 0)$, para todo $x \in Z$, existir uma $G$-homotopia $\bar{F}: Z \times I \rightarrow X$ tal que $p \circ \bar{F}=F$ e $\bar{F}(x, 0)=f(x)$, para todo $x \in Z$.

Logo, uma $G$-aplicação $f: X \rightarrow Y$ relacionando duas $G$-fibrações $(X, p, B)$ e $(Y, q, B)$ é dita uma $G$-aplicação entre $G$-fibrações que preserva fibra quando $q \circ f=p$. Daí, duas $G$-fibrações $(X, p, B)$ e $(Y, q, B)$ são $G$-equivalentes se existirem duas $G$-aplicações entre $G$-fibrações que preservam fibra $f: X \rightarrow Y$ e $g: Y \rightarrow X$ tais que $f \circ g$ é $G$ homotópica a $I d_{Y}$ (vamos denotar essa homotopia por $\phi_{1}$ ) e $g \circ f$ é $G$-homotópica a $I d_{X}$ (vamos denotar essa homotopia por $\phi_{2}$ ) de modo que o caminho $\phi_{i}(e, \bullet)$ está em uma única fibra, para todo $e \in Y$ quando $i=1$ ou para todo $e \in X$ quando $i=2$.

Por fim, dados $X$ e $B G$-espaços relacionados por uma $G$-aplicação $p: X \rightarrow B$, definimos uma $G$-aplicação de levantamento como sendo uma $G$-aplicação $\Lambda: \Omega_{p} \rightarrow$ $X^{I}$ tal que $\Lambda(e, \alpha)(0)=e$ e $p \circ \Lambda(e, \alpha)(t)=\alpha(t)$. Quando $\Lambda(e, p(e))=e$, onde $e \in X^{I}$ e $p(e) \in B^{I}$ estão sendo entendidos como os caminhos constantes, então chamaremos $\Lambda$ de $G$-aplicação de levantamento regular. 


\subsection{Resultados da Bibliografia}

Essa seção é dedicada aos resultados encontrados na bibliografia que serão necessários para as demonstrações que estão dispostas nos próximos capítulos. A ordem que adotamos é a apresentada na bibliografia desse texto. Na primeira subseção estão os resultados que usaremos no capítulo 2, e por ventura alguns também o serão no capítulo 3 . Na segunda subseção estão os resultados que usaremos no capítulo 3.

\subsubsection{Resultados da Bibliografia para Espaços e Aplicações}

É importante observar que os resultados aqui expostos não são traduções exatas dos originais, pois foram acondicionados as notações usadas nesse texto.

[1] Teorema VIII.B.4 (pg 123): Dados $K$ um complexo simplicial conexo, $s$ um p-simplexo maximal de $K, f:|K| \rightarrow|K|$ uma aplicação, $x \in|s|$ um ponto fixo isolado de $f$ e $U \subset|s|$ uma vizinhança de $x$ tal que $F i x(f) \cap \bar{U}=\{x\}$. Se o índice $I(f, U)=0$ então dado $\epsilon>0$, existe uma aplicação $f^{\prime}:|K| \rightarrow|K|$ com as seguintes propriedades:

1. $f(y)=f^{\prime}(y)$ para todo $y \in|K|-U$;

2. $d\left(f, f^{\prime}\right)<\epsilon$;

3. $F i x\left(f^{\prime}\right) \cap \bar{U}=\emptyset$.

[1] Lema VIII.C.3 (pg 128): Dados $K$ um complexo simplicial, $s_{1}, s_{2} \in K$ simplexos de dimensão maior do que 1 (um) tais que $s_{1} \cap s_{2}$ possui dimensão maior do que 0 (zero). Sejam $a \in\left|s_{1}\right|, b \in\left|s_{1} \cap s_{2}\right|$ e $f:|K| \rightarrow|K|$ uma aplicação tais que:

- $a$ é um ponto fixo isolado de $f$ com índice $I(f, U) \neq 0$, e Fix $(f) \cap[a, b]=\{a\}$;

- $f$ é uma aplicação proximal em $[a, b]$.

Então, existe $\epsilon>0$ e uma aplicação $f^{\prime}:|K| \rightarrow|K|$ com as seguintes propriedades:

1. existe uma homotopia $H:|K| \times I \rightarrow|K|$ entre $f^{\prime}$ e $f$ tal que $H(x, t)=H(x, 0)$ para todo $(x, t) \in(|K|-U(x, \eta)) \times I$;

2. Fix $\left(f^{\prime}\right) \cap \bar{U}([a, b], \epsilon)=\{c\}$ para algum $c \in U([a, b], \epsilon) \cap s_{2}$, onde $U([a, b], \epsilon)=\{x \in$ $|K| ; d(x, y)<\epsilon, y \in[a, b]\}$

3. $f^{\prime}$ é uma aplicação proximal em $\bar{U}([a, b], \epsilon)$

[2] Corolário 3.3.5 (pg 122): Sejam $K^{\prime}$ uma subdivisão de um complexo simplicial $K$ e $L$ um subcomplexo de $K$. Existe um único subcomplexo $L^{\prime}$ de $K^{\prime}$ que é uma subdivisão de $L$. 
[2] Corolário 3.3.11 (pg 124): Seja $(X, A)$ um par de poliedros. Então $A$ é um retrato forte por deformação de alguma vizinhança de $A$ em $X$.

[3] Proposição 8.12 (pg 83): Se $X$ for um subconjunto localmente compacto e localmente contrátil de $\mathbb{R}^{n}$ então existe um aberto $V$ de $\mathbb{R}^{n}$ que se retrai em $X$, assim $X$ é um ENR.

[6] Proposição II.8.6 (pg 158): Seja $G$ um grupo de Lie compacto. Se $X$ é um $G$-ENR e $i: X \rightarrow W$ é um $G$-mergulho em um $G$-módulo $W$, então $i(X)$ é um $G$-retrato de uma vizinhança.

Na Proposição anterior, temos que uma representação real (complexa) do grupo topológico $G$ no espaço vetorial real (complexo) de dimensão finita $V$ é uma ação contínua $G \times V \rightarrow V$ tal que, para todo $g \in G$, a translação a esquerda $L_{g}: V \rightarrow V$ dada por $L_{g}(v)=g v$ é uma transformação linear (real/complexa). Nesse caso, $V$ é um $G$-módulo.

[8] Lema 3.1 (pg 752): Sejam $K$ um complexo simplicial tal que todo simplexo maximal de $K$ tem dimensão maior ou igual a 2, o bordo de $S t(v)$ é conexo para todo vértice $v$ de $K$ e $K$ possui um 1-simplexo que é face de pelo menos três 2-simplexos. Para qualquer $\epsilon>0$, todo caminho em $|K|$ com pontos extremos diferentes e não situados em vértices de $K$ é $\epsilon$-homotópico a algum PL-caminho normal.

[8] Lema 5.1 (pg 757): Sejam $K$ um complexo simplicial tal que todo simplexo maximal de $K$ tem dimensão maior ou igual a 2, o bordo de $S t(v)$ é conexo para todo vértice $v$ de $K$ e $K$ possui um 1-simplexo que é face de pelo menos três 2-simplexos. Suponhamos que o PL-caminho normal $q$ satisfaz as seguintes condições:

1. existe um simplexo $\tau$, em um subconjunto aberto de $|K|$, e números $0<s_{1}<s_{3}<$ $s_{2}<1$, tais que $q(s) \in \partial(\tau)$ se e somente se $s=s_{1}$ ou $s=s_{2}, q\left(s_{3}\right) \in \tau$ e $q$ é linear em $\left[s_{1}, s_{3}\right]$ e em $\left[s_{3}, s_{2}\right]$;

2. $q\left(s_{1}\right)$ e $q\left(s_{2}\right)$ estão na realização geométrica do simplexo $\sigma_{1}$ com dimensão $\geq 1$, onde $\sigma_{1}$, por hipótese, é uma face de pelo menos dois simplexos maximais de $K$.

Suponhamos ainda que o simplexo $\sigma_{1}$ do item 2 é uma face 1-dimensional de pelo menos três 2-simplexos de $K$. Se dois caminhos especiais $p_{0}, p_{1}: I \rightarrow|K|$ são homotópicos, então eles são especialmente homotópicos.

[9] Lema 3.1 (pg 157): Seja $f: X \rightarrow X$ uma auto-aplicação de um poliedro conexo, compacto $X$ sem pontos de corte local e $A$ um subpoliedro. Suponha que $X-A$ não é uma dois variedade e que $A$ é contornável em $X$. Se $(\mathrm{C} 1)$ e $(\mathrm{C} 2)$ valerem então existe uma aplicação $g: X \rightarrow X$ homotópica a $f \operatorname{com} F i x(g)=A$, onde: 
(C1) Existe uma homotopia $\bar{H}: A \times I \rightarrow X$ entre $\bar{H}(\bullet, 0)=\left.f\right|_{A}$ e $\bar{H}(\bullet, 1)=i: A \hookrightarrow X$ (inclusão).

(C2) Para toda classe de pontos fixos essencial $F$ de $f$ existe um caminho $p: I \rightarrow X$ tal que $p(0) \in F, p(1) \in A$ e $\{p(t)\}$ é homotópica à $\bar{H}(p(t), t)$ (com pontos extremos fixados).

[11] Teorema 5.2 (pg 468): Seja $(X, A)$ um par de espaços onde $X$ é conexo por caminhos. Então $A$ é contornável em $X$ se e somente se $X-A$ é conexo por caminhos e o homomorfismo induzido pela inclusão $i_{*}: \pi_{1}(X-A) \rightarrow \pi_{1}(X)$ é sobrejetor.

Para o Lema 2.1 de [12] devemos entender que se $X$ é um $G$-espaço e $x \in X$ então a órbita de $x$ pela ação de $G$ é dada por $G(x)=\{g x \in X ; g \in G\}$. Por outro lado, dado um subgrupo fechado $H$ de $G$ temos que o espaço quociente $\frac{G}{H}$ munido da ação $\left(g, g^{\prime} H\right) \mapsto g g^{\prime} H$, para $g, g^{\prime} \in G$, é chamado espaço homogêneo.

Assim, se $G$ for um grupo compacto e $x \in X$ um ponto do $G$-espaço $X$ temos um homeomorfismo $\frac{G}{G_{x}} \rightarrow G(x)$ entre um espaço homogêneo e a órbita do ponto $x \in X$ dado por $g G_{x} \mapsto g x$. Desse modo, diremos que a órbita $G(x)$ possui tipo de órbita $\frac{G}{G_{x}}$. É importante observar que se duas órbitas possuem o mesmo tipo de órbita então seus pontos possuem o mesmo tipo de isotropia.

[12] Lema 2.1 (pg 278): Sejam $G$ um grupo de Lie compacto, $(X, A)$ um $G$-par de espaços tal que todas as órbitas em $X-A$ possuem o mesmo tipo de órbita $\frac{G}{H}$ e $f:(X, A) \rightarrow(X, A)$ uma $G$-aplicação. Seja $V$ uma $G$-vizinhança fechada de $A$ e $f^{H}$ : $X^{H} \rightarrow X^{H}$ a restrição de $f$ em $X^{H}$. Então qualquer $W H$-homotopia $f_{t}^{H}$, relativa a $V^{H}$, com $f_{0}^{H}=f^{H}$ se estende para uma $G$-homotopia $f_{t}$, relativa a $V$, com $f_{0}=f$. Além disso, se $f_{1}^{H}$ é livre de pontos fixos em $(X-A)^{H}$, então $f_{1}$ é livre de pontos fixos em $X-A$.

[13] Teorema 4.3 (pg 188): Sejam $G$ um grupo finito, $X$ a realização geométrica de um $G$-complexo simplicial e $f: X \rightarrow X$ uma $G$-aplicação. Dado $\epsilon>0$, existe uma $\epsilon$-homotopia equivariante $H: X \times I \rightarrow X$ tal que $H_{0}=f$ e $\left.H_{1}\right|_{X_{H}}$ possui uma quantidade finita de pontos fixos cada um no interior de algum simplexo maximal em $X^{H}$ para cada tipo de isotropia $(H)$ de $X$.

[15] Lema 1.3 (pg 1140): Sejam $G$ um grupo finito, $X$ a realização geométrica de um $G$-complexo simplicial, $X^{I}=\{\gamma: I \rightarrow X \mid \gamma$ é um caminho $\}$ o espaço dos caminhos em $X$ munido da topologia compacto aberto e com a $G$-ação dada por $(g \gamma)(t)=g(\gamma(t))$, para todo $\gamma \in X^{I}, \Delta=\{(x, x) \in X \times X\}$ a diagonal munida da ação diagonal $(g(x, x)=$ $(g x, g x)$ para todo $g \in G$ e todo $(x, x) \in \Delta)$ e $\eta(\Delta)=\{(x, y) \in X \times X \mid \sigma(x) \cap \sigma(y) \neq \emptyset\}$, onde $\sigma(x)$ é o único simplexo tal que $x \in|\sigma(x)|$.

Existe uma $G$-aplicação $\alpha: \eta(\Delta) \rightarrow X^{I}$ tal que: 
1. $\alpha(x, y)$ é um caminho de $x$ para $y$;

2. $\alpha(x, x)$ é o caminho constante em $x$;

3. o traçado de $\alpha(x, y)$ possui a forma $[x, z] \cup[z, y]$, onde $z$ depende de $x$ e $y$;

4. $\alpha(y, x)$ é o caminho reverso de $\alpha(x, y)$;

5. se $x \neq y$, então $\alpha(x, y)$ é um caminhos sem auto-interseção.

[18] Proposição 2.5 (pg 50): Sejam $G$ um grupo de Lie compacto, $X$ um $G$-ENR compacto, $A$ um $G$-subespaço de $X$ tal que existem um fechado $V \subset X$ que se retrai equivariantemente em $A$. Sejam $Y$ um compacto $G$-ENR e $f: X \rightarrow Y$ uma aplicação equivariante. Então, para todo $\epsilon>0$ existe uma vizinhança invariante $U$ de $A$ em $X$ e uma $\epsilon$-homotopia equivariante relativa a $A$ entre $f$ e uma aplicação $f^{\prime}$ tal que $\left.f^{\prime}\right|_{U}=\left.f^{\prime}\right|_{A} \circ r$, onde $r: V \rightarrow A$ é a $G$-retração de $V$ em $A$.

[18] Lema 3.1 (pg 52): Sejam $G$ um grupo finito, $X$ um $G$-espaço compacto e equivariantemente triangularizável, $A$ um $G$-subespaço fechado de $X$ tal que existem um fechado $V \subset X$ que se retrai equivariantemente sobre $A$ e $f:(X, A) \rightarrow(X, A)$ uma aplicação equivariante. Então existe uma aplicação equivariante $f^{\prime}$ equivariantemente homotópica a $f$ relativamente a $A$ tal que $\left.f^{\prime}\right|_{U}=\left.f^{\prime}\right|_{A} \circ r$, onde $r: V \rightarrow A$ é a $G$-retração de $V$ em $A$, e que possui uma quantidade finita de pontos fixos em $X-A$, cada um deles no interior de um simplexo maximal de $X$ para alguma triangularização equivariante de $X$.

[18] Lema 3.3 (pg 53): Sejam $G$ um grupo de Lie compacto de dimensão positiva, $M$ uma $G$-variedade compacta e suave, $A \subset M$ um fechado invariante de $M$ tal que existe uma vizinhança invariante $V \subset M$ que se retrai equivariantemente sobre $A$ e a ação de $G$ em $M-A$ é livre. Qualquer aplicação equivariante $f:(M, A) \rightarrow(M, A)$ é equivariantemente homotópica, relativamente a $A$, a uma $G$-aplicação $f^{\prime}$ tal que $f^{\prime}$ não possui pontos fixos em $M-A$.

[20] Teorema 1.3 (pg 2): Seja $f: X \rightarrow X$ uma auto-aplicação em um poliedro conexo, compacto e sem pontos de corte local e seja $\Phi$ um subpoliedro fechado de $X$ satisfazendo:

- $X-\Phi$ não é uma 2-variedade;

- $\Phi$ é contornável em $X$;

(C1) existe uma homotopia $\bar{H}: \Phi \times I \rightarrow X$ entre $\bar{H}(\bullet, 0)=\left.f\right|_{\Phi}$ e $\bar{H}(\bullet, 1)=i: \Phi \hookrightarrow X$ a inclusão; 
(C2) para toda classe de pontos fixos essencial $F$ de $f$ existe um caminho $p: I \rightarrow X$ tal que $p(0) \in F, p(1) \in \Phi$ e $\{p(t)\}$ é homotópica à $\bar{H}(p(t), t)$ com pontos extremos fixados.

Então para todo subconjunto fechado $T$ de $\Phi$ que intercepta todas as componentes conexas de $\Phi$, existe uma aplicação $g$ homotópica a $f \operatorname{com} F i x(g)=T$. Em particular, se $\Phi$ é conexo, então todo subconjunto de $\Phi$ (incluindo o próprio $\Phi$ ) é o conjunto de pontos fixos de uma aplicação homotópica a $f$.

[20] Teorema 2.2 (pg 3): Se $X$ for um poliedro conexo e compacto, $\Phi \subset X$ for um subconjunto localmente contrátil e fechado. Se $\Phi$ for contornável em $X$ então existe uma vizinhança aberta $V$ de $\Phi$ em $X$ tal que $V$ é contornável em $X$.

[22] Proposição 9.3 (pg 503): Sejam $G$ um grupo de Lie compacto, $Y$ um $G$-ANR e $(X, A)$ um $G$-par metrizável então toda função $h: X \times\{0\} \cup A \times I \rightarrow Y$ possui uma $G$-extensão $H: X \times I \rightarrow Y$.

Para os resultados de [23] devemos entender o que é uma triangularização suave. Se $X$ for um subconjunto de $|K|$ então denotamos por $N(X, K)$ o subcomplexo de $K$ formado por todos os simplexos de $K$ cujas realizações geométricas interceptam $X$. Dado um grupo finito $G$ dizemos que um homeomorfismo $\tau$ de $G$ no espaço das matrizes ortogonais $n$-dimensionais $O(n)$ é uma representação ortogonal de $G$ e denotamos por $\mathbb{R}^{n}(\tau)$ o espaço euclidiano $\mathbb{R}^{n}$ munido com a $G$-ação induzida por $\tau$.

Dado algum espaço de representação ortogonal para $G \mathbb{R}^{p}(\sigma)$, uma $G$-aplicação $f$ : $|K| \rightarrow \mathbb{R}^{p}(\sigma)$ é suave se para todo simplexo $s$ de $K$ a aplicação $\left.f\right|_{|s|}:|s| \rightarrow \mathbb{R}^{p}(\sigma)$ é diferenciável de classe $\mathcal{C}^{\infty}$ e para cada $b \in|s|$ as aplicações de $d f_{b}$ definidas em todas as realizações geométricas dos simplexos que contém $b$ concordam nas realizações geométricas das faces em comum e portanto geram uma aplicação bem definida $d f_{b}:|N(b, K)| \rightarrow$ $\mathbb{R}^{p}(\sigma)$. Além disso, $|N(b, K)|$ é um $G_{b}$-espaço e $d f_{b}(h x)=h d f_{b}(x)$.

Seja $M$ uma $G$-variedade suave, então $M$ mergulha em algum $\mathbb{R}^{p}(\sigma)$ como uma $G$ subvariedade suave. Daí, [23] chama uma $G$-aplicação suave $f:|K| \rightarrow M$ de uma triangularização equivariante suave se $f(|K|)=M$ e a $G_{b^{-}}$aplicação $d f_{b}:|N(b, K)| \rightarrow$ $\mathbb{R}^{p}\left(\sigma \mid G_{b}\right)$ é injetiva para todo $b \in|K|$, onde $\mathbb{R}^{p}\left(\sigma \mid G_{b}\right)$ denota o espaço de representação ortogonal de $G_{b}$ dado pela representação $\left.\sigma\right|_{G_{b}}: G_{b} \rightarrow O(p)$.

[23] Teorema (pg 199): Sejam $G$ um grupo finito e $M$ uma $G$-variedade suave, com ou sem bordo. Então:

1. existe um complexo simplicial $K$ e uma $G$-triangularização suave $h:|K| \rightarrow M$;

2. se $h:|K| \rightarrow M$ e $h_{1}:|L| \rightarrow M$ são $G$-triangularizações suaves de $M$ então existem $G$-subdivisões $K^{\prime}$ e $L^{\prime}$ de $K$ e $L$,respectivamente, tais que $K^{\prime}$ e $L^{\prime}$ são $G$-isomorfos; 
[23] Teorema 3.6 (pg 216): Sejam $G$ um grupo finito, $M$ uma $G$-variedade suave e $N$ uma $G$-subvariedade fechada e suave de $M$. Então existe uma triangularização suave e equivariante $f:\left(|K|,\left|K_{0}\right|\right) \rightarrow(M, N)$.

[23] Teorema 3.7 (pg 216): Sejam $G$ um grupo finito, $M$ uma $G$-variedade suave, $N$ uma $G$-subvariedade fechada e suave de $M$ e $f:\left(|K|,\left|K_{0}\right|\right) \rightarrow(M, N)$ e $h:\left(|L|,\left|L_{0}\right|\right) \rightarrow$ $(M, N)$ duas triangularizações suaves e equivariantes de $(M, N)$. Então, existem aplicações equivariantes $f^{\prime}:\left(\left|K^{\prime}\right|,\left|K_{0}^{\prime}\right|\right) \rightarrow(M, N)$ e $h^{\prime}:\left(\left|L^{\prime}\right|,\left|L_{0}^{\prime}\right|\right) \rightarrow(M, N)$ para $f$ e $h$, respectivamente, tais que $f^{\prime}$ e $h^{\prime}$ são triangularizações suaves e equivariantes de $(M, N)$ e $\left(h^{\prime}\right)^{-1} \circ f^{\prime}:\left(|K|,\left|K_{0}\right|\right) \rightarrow\left(|L|,\left|L_{0}\right|\right)$ é um $G$-homeomorfismo simplicial.

\subsubsection{Resultados da Bibliografia para Fibrados e Fibrações}

Essa subseção é dedicada aos resultados da bibliografia que usaremos em nossas demonstrações do terceiro capítulo e que ainda não formam alocados nesse texto.

[2] Corolário 2.7.13 (pg 96): Se $(E, p, B, F)$ é um fibrado com espaço base $B$ Hausdorff e paracompacto, então $(E, p, B)$ é uma fibração.

[4] Teorema XX.2.2 (pg 396): (E,p,B) é uma fibração (regular) se, e somente se, existe uma aplicação de levantamento (regular).

[4] Teorema XX.2.7 (pg 398): Seja $(E, p, B)$ uma fibração regular. Assuma que $B$ é contrátil para um ponto $b_{0} \in B$, e seja $F=p^{-1}\left(b_{0}\right)$. Então $(E, p, B)$ é equivalente a fibração $(B \times F, q, B)$, onde $q$ é a projeção no primeiro fator.

[5] Teorema III.4.1 (pg 65): Todo fibrado $(E, p, B)$ possui a propriedade de levantamento de homotopias para qualquer espaço triangularizável.

[9] Teorema 4.2 (pg 160): Sejam $f: X \rightarrow X$ uma aplicação no espaço $A N R$ compacto e conexo $X$ e $A$ um subconjunto não vazio e fechado de $X$.

1. Se o número de Nielsen de $f$ for $0, \mathcal{N}(f)=0$, ou se $X$ for simplesmente conexo, então $(C 2)$ é válida;

2. Se $A \subset F i x(f)$ e $A$ intersepta todas as classes de pontos fixos essenciais de $f$, então $(C 1)$ e $(C 2)$ são válidas;

3. Se Fix $(f) \subset A$ e $(C 1)$ é satisfeita por uma homotopia $\bar{H}: A \times I \rightarrow X$ tal que $\bar{H}(x, t)=x$ para todo $(x, t) \in F i x(f) \times I$, então $(C 2)$ é válida.

Onde, 
(C1) existe uma homotopia equivariante $\bar{H}: A \times I \rightarrow X$ entre $\bar{H}(\bullet, 0)=\left.f\right|_{A}$ e $\bar{H}(\bullet, 1)=$ $i: A \hookrightarrow X$ a inclusão;

(C2) para toda classe de pontos fixos essencial $F$ de $f$ existe um caminho $p: I \rightarrow X$ tal que $p(0) \in F, p(1) \in A$ e $\{p(t)\}$ é homotópica à $\bar{H}(p(t), t)$ com pontos extremos fixados.

[19] Lema 2.1 (pg 44): Sejam $\mathfrak{F}=(E, p, B ; Y)$ um fibrado onde $E, B$ e $Y$ são poliedros conexos, $f: E \rightarrow E$ uma aplicação que preserva fibra e $A$ um subconjunto de $X$ tal que:

$(C 1)_{\mathfrak{F}}$ existe uma homotopia parcial que preserva fibra $H_{A}:(E \times\{0\}) \cup(A \times I) \rightarrow X$ tal que $H_{A}(\bullet, 0)=f$ e $H_{A}(\bullet, 1)=i: A \hookrightarrow E$ (inclusão);

$(C 2)_{\mathfrak{F}}$ toda classe de pontos fixos essencial $F$ de $f$ está conectada a $A$ por um caminho $\gamma: I \rightarrow X$ tal que $\gamma(0) \in F, \gamma(1) \in A$ e $\{\gamma(t)\}$ é homotópica à $\{f \circ \gamma\} *\left\{H_{A}(\gamma(1), t)\right\}$ com pontos extremos fixados.

Então, para toda classe de pontos fixos essencial $F$ de $f$ existe um caminho $\bar{\alpha}: I \rightarrow B$ $\operatorname{com} \bar{\alpha}(0) \in p(F), \bar{\alpha}(1) \in p(A)$ e $\{\bar{\alpha}(t)\}$ é homotópica à $\{\bar{f} \circ \bar{\alpha}(t)\} *\left\{\bar{H}_{A}(p \circ \alpha(1), t)\right\}$, com pontos extremos fixados.

[19] Teorema 4.1 (pg 49): Seja $\mathfrak{F}=(E, p, B, Y)$ uma fibrado onde $E, B$ e $Y$ são poliedros conexos e $B$ e $Y$ não possuem pontos de corte local.

Seja $A$ um subconjunto fechado e localmente contrátil de $E$ tal que $(E, A)$ é um par de fibrados para $\mathfrak{F}, p(A)$ é um subconjunto fechado e localmente contrátil de $B$, cada componente $p(A)_{j}$ de $p(A)$ é contrátil em $B, p(A)$ é contornável em $B$ e $B-p(A)$ não é uma 2-variedade.

Seja $f: E \rightarrow E$ uma aplicação que preserva fibra tal que:

$(C 1)_{\mathfrak{F}}$ existe uma homotopia parcial que preserva fibra $H_{A}:(E \times\{0\}) \cup(A \times I) \rightarrow E$ tal que $H_{A}(\bullet, 0)=f$ e $H_{A}(\bullet, 1)=i: A \hookrightarrow E($ inclusão);

$(C 2)_{\mathfrak{F}}$ toda classe de pontos fixos essencial $F$ de $f$ está conectada a $A$ por um caminho $\gamma: I \rightarrow E$ tal que $\gamma(0) \in F, \gamma(1) \in A$ e $\{\gamma(t)\}$ é homotópica à $\{f \circ \gamma\} *\left\{H_{A}(\gamma(1), t)\right\}$ com pontos extremos fixados.

Supondo ainda que para todo subfibrado $Y_{j}$ de $A$ temos que $Y_{j}$ é um subespaço fechado, localmente contrátil e contornável em $Y$ tal que $Y-Y_{j}$ não é uma 2-variedade e que $A$ intercepta todas as classes de pontos fixos essenciais de $f_{b_{j}}: p^{-1}\left(b_{j}\right) \rightarrow p^{-1}\left(b_{j}\right)$ para pelo menos um $b_{j}$ na componente $p(A)_{j}$. Se $Z$ é um subconjunto fechado e fibrado de $A$ que intercepta todas as componentes de $A$, então existe uma aplicação $g: E \rightarrow E$ que é homotópica a $f$ por uma homotopia que preserva fibra tal que $F i x(g)=Z$. 
[24] Teorema 2.1 (pg 239): Sejam $\Gamma=(E, p, B)$ um fibrado e $(X, A)$ uma par de espaços métricos com $A$ fechado em $X$. Suponha que exista uma aplicação $G:(X \cup\{0\}) \cup$ $(A \times I) \rightarrow E$ tal que $p \circ G(x, t)=p \circ G(x, 0)$, para $x \in A$ e $0 \leq t \leq 1$. Então, se $E$ for um ANR ou se $(X, A)$ for um par ANR então $G$ pode ser estendida para uma homotopia $H: X \times I \rightarrow E$ tal que $p \circ H(x, t)=p \circ H(x, 0)$, para $x \in X$ e $0 \leq t \leq 1$. 


\section{Capítulo 2}

\section{Realização de Conjuntos de Pontos Fixos}

Como já foi dito anteriormente, apresentaremos neste trabalho versões do resultado obtido por H. Schirmer em [9] no contexto equivariante. Desse modo, o objetivo desse segundo capítulo é obter uma generalização do Lema 3.1 de [9] no contexto de variedades equivariantes.

Ampliaremos o trabalho usando a ideia apresentada por C. L. Soderlund em [20] que possibilita realizar, mediante certas hipóteses, subconjuntos localmente contráteis como o conjunto de pontos fixos de uma aplicação homotópica a uma aplicação dada.

Os subconjuntos localmente contráteis constituem uma classe grande de subconjuntos em uma variedade e está constatado em [3] (Proposição 8.12 da página 83) que todo subconjunto de um espaço euclidiano que seja localmente compacto e localmente contrátil é um ANR. Também é conhecido que todo subespaço fechado de um espaço ANR possui uma vizinhança que se retrai fortemente.

Daí, C. L. Soderlund observou que dados um poliedro conexo e compacto $X$, uma aplicação $f: X \rightarrow X$, um subconjunto fechado $\Phi$ de $X$ tal que $f$ satisfaz $(C 1)$ e $(C 2)$ para $\Phi$ e um subconjunto $B$ de $X$ tal que $\Phi \subset B$ e $B$ se retrai fortemente em $\Phi$ temos que $f$ satisfaz $(C 1)$ e $(C 2)$ para $B$.

Na primeira seção desse capítulo apresentaremos condições análogas as dadas por H. Schirmer em [9], provaremos sua necessidade e apresentaremos alguns exemplos. Na segunda e na terceira seções faremos alguns lemas e procedimentos que usaremos na demonstração da generalização do Lema 3.1 de [9] situada na quarta seção. Na quarta seção também faremos alguns corolários que trazem contextos particulares. 


\subsection{Exemplos}

Vamos dedicar essa primeira seção para apresentar os primeiros exemplos a respeito do que será feito ao longo desse capítulo. H. Schirmer provou o seguinte resultado:

Teorema 4.5 de [9]: Seja $f: \mathbb{S}^{n} \rightarrow \mathbb{S}^{n}$ uma auto-aplicação entre $n$-esferas com $n \geq 2$. Então, para todo $A$ subconjunto próprio fechado e não vazio de $\mathbb{S}^{n}$ existe uma auto-aplicação $g: \mathbb{S}^{n} \rightarrow \mathbb{S}^{n}, g \in[f]$, tal que $\operatorname{Fix}(g)=A$.

No resultado acima $[f]$ representa a classe de homotopia de $f$. Observamos que esse resultado não é válido quando se insere uma ação de grupo e o exemplo abaixo ilustrará que essa diferença entre os casos equivariante e não equivariante não está relacionada apenas com a invariância do conjunto de pontos fixos.

Exemplo 1: Tomemos $G=\mathbb{Z}_{2}, X=\mathbb{S}^{2}$, a ação será $\xi(x, y, z) \mapsto(-x,-y, z)$, assim $X^{G}=\{N=(0,0,1), S=(0,0,-1)\}, A=\left\{(x, y, 0) \in \mathbb{S}^{2}\right\}$ e $f=I d$ a aplicação identidade. Para esses objetos não existe uma $G$-homotopia $H$ entre $f$ e uma $G$-aplicação $h: X \rightarrow X$ tal que $F i x(h)=A$.

De fato, se existisse tal aplicação teríamos que $h(N)=S$ e que $h(S)=N$, pois $h$ é equivariante e portanto leva $X^{G}$ em $X^{G}$. Daí, $p: I \rightarrow X^{G}$ dada por $p(t)=H^{G}(N, t)$ é um caminho tal que $p(0)=f(N)=N$ e $p(1)=h(N)=S$ o que é um absurdo.

É muito interessante observar que para esse exemplo a forma dos objetos não é mais importante do que a posição dos mesmos, isto é, substituindo $A$ por $A^{\prime}=\left\{(x, 0, z) \in \mathbb{S}^{2}\right\}$ somos capazes de construir uma $G$-homotopia entre a identidade e uma $G$-aplicação $h$ que fixa $A^{\prime}$. Usaremos coordenadas esféricas para facilitar nas contas. Para $0 \leq \psi \leq \pi$ e $0 \leq \theta \leq 2 \pi$ temos que:

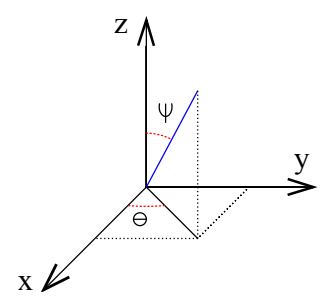

Figura 2.1: Coordenadas Esféricas

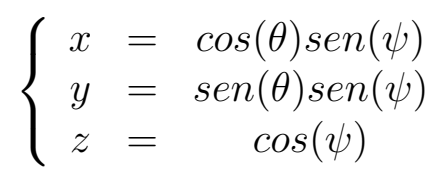

Tomemos $0.001>\epsilon>0$ para a seguinte $G$-homotopia :

$$
\begin{gathered}
H(t, \cos (\theta) \operatorname{sen}(\psi), \operatorname{sen}(\theta) \operatorname{sen}(\psi), \cos (\psi))= \\
(\cos (\theta+t \epsilon \operatorname{sen}(\theta)) \operatorname{sen}(\psi), \operatorname{sen}(\theta+t \epsilon \operatorname{sen}(\theta)) \operatorname{sen}(\psi), \cos (\psi)) .
\end{gathered}
$$

O exemplo anterior ilustrou que nem todo subconjunto $\mathbb{Z}_{2}$-invariante, fechado e não vazio de $\mathbb{S}^{n}$ pode ser realizado como o conjunto de pontos fixos de uma dada $\mathbb{Z}_{2}$-autoaplicação de $\mathbb{S}^{n}$, mesmo para aplicações simples como é o caso da identidade. O que demonstra que 
o Teorema 4.5 de [9] que H. Schirmer obteve para o caso não equivariante não é válido para o caso equivariante.

[9] é um artigo que dá condições necessárias e suficientes para realização de conjuntos de pontos fixos de auto aplicações dadas. O objetivo desse capítulo é generalizar para o caso equivariante alguns resultados de [9] e faremos isso usando algumas técnicas de [20]. Para exemplificar, uma possível generalização do Lema 3.1 de [9] é dada por:

Lema: Dados $G$ um grupo de Lie compacto, $X$ uma $G$-variedade suave e compacta, $f: X \rightarrow X$ uma $G$-aplicação, $A$ uma $G$-subvariedade de $X$, fechada e não vazia tais que para $W K$ finito temos que $\operatorname{dim}\left(X^{K}\right) \geq 3, \operatorname{dim}\left(X^{K}\right)-\operatorname{dim}\left(X^{K}-X_{K}\right) \geq 2$ e $A^{K}$ é contornável em $X^{K}$, onde $(K) \in I s o(X)$. Suponhamos que:

(C1) exista uma $G$-aplicação $\bar{H}:(X \times\{0\}) \cup(A \times I) \rightarrow X$ tal que $\bar{H}(\bullet, 0)=f$ e $\bar{H}(\bullet, 1)=i: A \hookrightarrow X$ (inclusão).

(C2) sempre que $W K$ for finito, toda $W K$-classe de pontos fixos essencial $F$ de $f^{K}$ está conectada a $A^{K}$ por um caminho $p: I \rightarrow X^{K} \operatorname{com} p(0) \in F$ e $p(1) \in A^{K}$ e tal que $\{p(t)\}$ é homotópica à $\left\{f^{K} \circ p\right\} *\left\{\bar{H}^{K}(p(1), t)\right\}$, com pontos extremos fixados.

Então $f$ é equivariantemente homotópica a uma $G$-aplicação $h: X \rightarrow X$ tal que $\operatorname{Fix}(h)=A$.

O Lema acima será provado mais adiante. Observamos que são usadas hipóteses especiais para os tipos de isotropia $(K)$ de $X$ tais que $W K$ é finito, nesses casos temos que $\operatorname{dim}\left(X^{K}\right) \geq 3, \operatorname{dim}\left(X^{K}\right)-\operatorname{dim}\left(X^{K}-X_{K}\right) \geq 2$ e $A^{K}$ é contornável em $X^{K}$. Essas hipóteses aparecerão em vários resultados que provaremos ao longo desse trabalho inclusive nas generalizações que propomos por isso devemos entende-las melhor.

A técnica que usaremos quando $W K_{i}$ é finito consiste em triangularizar $X_{i}^{K_{i}}$ de modo $W K_{i}$-equivariante, onde o índice $i$ vem de uma ordem admissível em $I s o(X)$. A partir dessa triangularização criamos caminhos que levam as $W K_{i}$-classes de pontos fixos essenciais a $A$. Contudo, esses caminhos não podem se intersectar, e para que isso ocorra precisamos das hipóteses de dimensão do espaço $X_{i}^{K_{i}}$.

Quando $W K_{i}$ é um grupo de Lie compacto com dimensão positiva é conhecido que $f^{K_{i}}$ é $W K_{i}$-homotópica a uma aplicação livre de pontos fixos em $X_{i}^{K_{i}}-X_{i-1}^{K_{i}}$ (veja Lema 3.3 de [18]). Isso faz com que não existam $W K_{i}$-classes de pontos fixos essenciais de $f^{K_{i}}$ em $X_{i}^{K_{i}}-X_{i-1}^{K_{i}}$, e assim, as hipóteses de dimensão do espaço $X_{i}^{K_{i}}$ e a condição $(C 2)$ se tornam desnecessárias nesse caso.

Agora, vamos analisar um pouco mais as condições $C 1$ e $C 2$ que demos. Começaremos testando-as no Exemplo 1 dado anteriormente.

No Exemplo 1 podemos facilmente verificar $C 1$ porque usamos a identidade mas $C 2$ não é válido. De fato, não existe nenhum caminho em $X^{G}$ conectando $X^{G}$ a $A$ e portanto $C 2$ não é válido. Observamos que é importante verificar essas condições quando queremos 
realizar um conjunto como o conjunto de pontos fixos de uma $G$-aplicação $G$-homotópica a uma $G$-aplicação dada, pois o próximo lema prova a necessidade delas.

Lema 2.1 Dados $G$ um grupo de Lie compacto, $X$ um $G$-espaço $G$-ANR, $f: X \rightarrow X$ uma $G$-aplicação e $A$ um $G$-subespaço de $X$, fechado e não vazio. Suponhamos que $f$ é equivariantemente homotópica a uma $G$-aplicação $h: X \rightarrow X$ tal que Fix $(h)=A$ então as seguintes condições acontecem:

(C1) existe uma $G$-aplicação $\bar{H}:(X \times\{0\}) \cup(A \times I) \rightarrow X$ tal que $\bar{H}(\bullet, 0)=f$ e $\bar{H}(\bullet, 1)=i: A \hookrightarrow X($ inclusão $)$.

(C2) sempre que $W K$ for finito, toda $W K$-classe de pontos fixos essencial $F$ de $f^{K}$ está conectada a $A^{K}$ por um caminho $p: I \rightarrow X^{K} \operatorname{com} p(0) \in F$ e $p(1) \in A^{K}$ e tal que $\{p(t)\}$ é homotópica à $\left\{f^{K} \circ p\right\} *\left\{\bar{H}^{K}(p(1), t)\right\}$, com pontos extremos fixados.

Prova do Lema 2.1: Seja $H: X \times X \rightarrow X$ a G-homotopia que começa em $f$ e termina em $h$. Então

$$
\bar{H}=\left.H\right|_{(X \times\{0\}) \cup(A \times I)}:(X \times\{0\}) \cup(A \times I) \rightarrow X
$$

é tal que $\bar{H}(\bullet, 0)=f$ e $\bar{H}(\bullet, 1)=i: A \hookrightarrow X$ (inclusão), e assim, $(C 1)$ é válido.

Se $F$ for uma $W K$-classe de pontos fixos essencial de $f^{K}$, para $W K$ finito, então existe um caminho $p: I \rightarrow X^{K}$ tal que $p(0) \in F$ e $p(1) \in J$, onde $J \subset A^{K}$ é a $W K$-classe de pontos fixos essencial de $h^{K}$ que está $H^{K}$-relacionada com $F$ e $\{p(t)\}$ é homotópica à $\left\{\bar{H}^{K}(p(t), t)\right\}$ (com pontos extremos fixados).

Como $\left\{\bar{H}^{K}(p(t), t)\right\}$ é homotópica (com pontos extremos fixados) à:

$$
\underbrace{\left\{\bar{H}^{K}(p(t), 0)\right\}}_{=\{f \circ p(t)\}} *\left\{\bar{H}^{K}(p(1), t)\right\},
$$

pela homotopia de caminhos dada por:

$$
\begin{array}{rlc}
Y:\left(X^{K} \times I\right) \times I & \rightarrow & X^{G} \times I \\
((x, t), s) & \mapsto \bar{H}^{K}(p(t),(1-s) t) * \bar{H}^{K}(p(1), 1+s(t-1)),
\end{array}
$$

temos que $(C 2)$ está satisfeito.

Para ilustrar o Lema 2.1 vamos testar as condições $C 1$ e $C 2$ em alguns exemplos. No próximo $C 1$ não será válido mas $C 2$ será.

Exemplo 2: Tomemos $G=\mathbb{Z}_{2}, X=\mathbb{S}^{3}$, a ação será $\xi(x, y, z, w) \mapsto(x, y, z,-w)$ (reflexão com relação ao hiperplano $w=0$ ), assim $X^{G}=\left\{(x, y, z, 0) \in \mathbb{S}^{3}\right\}=\mathbb{S}^{2}, A=X^{G}$ e $f=-I d$ a aplicação antípoda. Dessa vez, $C 2$ é obviamente verdadeira, pois $f$ é livre de pontos fixos. 
Por outro lado, toda $G$-aplicação preserva filtração assim não é possível encontrar uma $G$-aplicação $\bar{H}:(X \times\{0\}) \cup(A \times I) \rightarrow X$ tal que $\bar{H}(\bullet, 0)=f$ e $\bar{H}(\bullet, 1)=i: A \hookrightarrow X$ (inclusão), pois $f^{G}: X^{G}=\mathbb{S}^{2} \rightarrow X^{G}=\mathbb{S}^{2}$ tem grau -1 enquanto que o grau de $i^{G}: X^{G}=$ $\mathbb{S}^{2} \rightarrow X^{G}=\mathbb{S}^{2}$ é 1 .

Com os objetos do Exemplo 1 só é possível realizar conjuntos que intersectam $X^{G}$, mas isso não é uma exigência. No próximo exemplo trabalharemos um pouco mais a relação entre $A$ e $X^{G}$.

Exemplo 3: Tomemos $G=\mathbb{Z}_{2}, X=\mathbb{S}^{2} \times \mathbb{S}^{1}$, a ação será o conjugado na segunda coordenada:

$$
\xi((a, b, c), \cos (x)+i \operatorname{sen}(x)) \mapsto((a, b, c), \cos (x)-i \operatorname{sen}(x)) .
$$

Assim $X^{G}=\mathbb{S}^{2} \times\{-1,1\}$. Usaremos a aplicação antípoda em $\mathbb{S}^{2}$ e a identidade em $\mathbb{S}^{1}$ :

$$
f((a, b, c), \cos (x)+i \operatorname{sen}(x))=((-a,-b,-c), \cos (x)+i \operatorname{sen}(x))
$$

para essa aplicação realizaremos $A$ com algumas formas diferentes. Primeiramente, fixaremos $A_{1}$ que não intercepta $X^{G}$, isso é possível porque $f$ não possui pontos fixos em $X^{G}$. Seja $A_{1}=\left\{(a, b, 0) \in \mathbb{S}^{2}\right\} \times\{-i, i\}=\mathbb{S}^{1} \times\{i,-i\}$, daí, a homotopia será:

$$
\begin{gathered}
H_{1}((\cos (\theta) \operatorname{sen}(\psi), \operatorname{sen}(\theta) \operatorname{sen}(\psi), \cos (\psi)), \cos (x)+i \operatorname{sen}(x), t)= \\
((-\cos (\theta+t|\operatorname{sen}(x)| \pi) \operatorname{sen}(\psi),-\operatorname{sen}(\theta+t|\operatorname{sen}(x)| \pi) \operatorname{sen}(\psi),-\cos (\psi)), \cos (x)+i \operatorname{sen}(x)) .
\end{gathered}
$$

Agora, criaremos uma situação onde existe intersecção entre $A_{2}$ e $X^{G}$ mas de forma que um não contenha o outro. Usaremos $A_{2}=\mathbb{S}^{1} \times \mathbb{S}^{1}$, para esse caso a $G$-homotopia é:

$$
\begin{gathered}
H_{2}((\cos (\theta) \operatorname{sen}(\psi), \operatorname{sen}(\theta) \operatorname{sen}(\psi), \cos (\psi)), \cos (x)+i \operatorname{sen}(x), t)= \\
((-\cos (\theta+t(1-|\cos (\psi)|) \pi) \operatorname{sen}(\psi),-\operatorname{sen}(\theta+t(1-|\cos (\psi)|) \pi) \operatorname{sen}(\psi),-\cos (\psi)), \\
\cos ((1-t) x+t x(1-|\cos (\psi)|))+i \operatorname{sen}((1-t) x+t x(1-|\cos (\psi)|))) .
\end{gathered}
$$

Observemos que esse exemplo é tão rico com relação as possíveis formas de $A$ que é possível fixar até um conjunto $A$ finito. De fato, tomemos:

$$
A_{3}=\{((-1,0,0),-i),((-1,0,0), i),((1,0,0),-i),((1,0,0), i)\},
$$

para esse caso a $G$-homotopia é:

$$
\begin{gathered}
H_{3}((\cos (\theta) \operatorname{sen}(\psi), \operatorname{sen}(\theta) \operatorname{sen}(\psi), \cos (\psi)), \cos (x)+i \operatorname{sen}(x), t)= \\
((-\cos (\theta+t|\operatorname{sen}(x)||\cos (\theta)| \pi) \operatorname{sen}(\psi),-\operatorname{sen}(\theta+t|\operatorname{sen}(x) \| \cos (\theta)| \pi) \operatorname{sen}(\psi),-\cos (\psi)), \\
\cos (x)+i \operatorname{sen}(x)) .
\end{gathered}
$$

Um outro aspecto que torna esse exemplo rico é o fato de existirem subconjuntos invariantes que não podem ser fixados. Por exemplo $A_{4}=X^{G}$. 
De fato, se existisse uma $G$-homotopia entre $f$ e uma aplicação $h: X \rightarrow X$ tal que Fix $(h)=A_{4}$. Então, como $h$ é uma $G$-aplicação, a aplicação $\left.h^{G}\right|_{\mathbb{S}^{2} \times\{1\}}=i d$ : $\mathbb{S}^{2} \times\{1\} \rightarrow \mathbb{S}^{2} \times\{1\}$ (aplicação identidade) que tem grau 1 seria homotópica a aplicação $\left.f^{G}\right|_{\mathbb{S}^{2} \times\{1\}}=-i d: \mathbb{S}^{2} \times\{1\} \rightarrow \mathbb{S}^{2} \times\{1\}$ (aplicação antípoda) que tem grau -1, o que é um absurdo.

Por fim, um exemplo onde $G$ não é finito.

Exemplo 4: Após o exemplo 3 fica fácil tomar os seguintes objetos: $G=\mathbb{S}^{1}, X=$ $\mathbb{S}^{2} \times \mathbb{S}^{1}$, a ação será a multiplicação na segunda coordenada:

$$
(\cos (y)+i \operatorname{sen}(y))((a, b, c), \cos (x)+i \operatorname{sen}(x)) \mapsto((a, b, c), \cos (x+y)+i \operatorname{sen}(x+y)) .
$$

Usaremos a $G$-aplicação $f$ do exemplo 3 :

$$
f((a, b, c), \cos (x)+i \operatorname{sen}(x))=((-a,-b,-c), \cos (x)+i \operatorname{sen}(x)) .
$$

As $\mathbb{Z}_{2}$-homotopias $H_{1}$ e $H_{3}$ não são $\mathbb{S}^{1}$-homotopias para esse caso mas $H_{2}$ é uma $\mathbb{S}^{1}$-homotopia. Dessa forma, ainda é possível fixar o conjunto $A_{2}$ por aplicações $\mathbb{S}^{1}$ homotópicas a $f$ (basta usar $H_{2}$ ). Contudo, os conjuntos $A_{1}$ e $A_{2}$ não podem mais ser fixados por aplicações $\mathbb{S}^{1}$-homotópicas a $f$, pois se $h$ é $\mathbb{S}^{1}$-equivariante e:

$$
((a, b, c), \cos (x)+i \operatorname{sen}(x)) \in \mathbb{S}^{2} \times \mathbb{S}^{1}
$$

é um ponto fixo de $h$ então $\{(a, b, c)\} \times \mathbb{S}^{1} \subset F i x(h)$.

De fato, dados $((a, b, c), \cos (y)+i \operatorname{sen}(y)) \in\{(a, b, c)\} \times \mathbb{S}^{1}$ e $z=y-x$ temos que:

$$
\begin{aligned}
h((a, b, c), \cos (y)+i \operatorname{sen}(y)) & =h((\cos (z)+i \operatorname{sen}(z))((a, b, c), \cos (x)+i \operatorname{sen}(x))) \\
& =(\cos (z)+i \operatorname{sen}(z))(h((a, b, c), \cos (x)+i \operatorname{sen}(x))) \\
& =(\cos (z)+i \operatorname{sen}(z))((a, b, c), \cos (x)+i \operatorname{sen}(x)) \\
& =((a, b, c), \cos (y)+i \operatorname{sen}(y)) .
\end{aligned}
$$




\section{$2.2 \quad$ Resultados Preliminares}

Faremos nessa seção algumas observações que serão necessárias nas demonstrações das generalizações dos resultados de [9] anteriormente citados. A primeira está relacionada a um trecho escrito em [17].

Proposição 2.2 : Sejam $G$ um grupo de Lie compacto e $(X, A)$ um par G-ENR. Então, existem uma vizinhança invariante $V \subset X$ de $A$ e uma $G$-retração $r: V \rightarrow A$.

Prova da Proposição 2.2: $(X, A)$ é um par $G$-ENR então existe $G$-mergulho $i$ : $X \hookrightarrow E$ de $X$ em um $G$-espaço euclidiano $E$ e $\left.i\right|_{A}: A \hookrightarrow E$ é um $G$-mergulho de $A$ em $E$.

Pela Proposição II.8.6 de [6] existem uma vizinhança $G$-invariante $W \subset E$ de $i(A)$ e uma $G$-retração $\tilde{r}: W \rightarrow i(A)$. Assim $W \cap i(X)$ é uma vizinhança $G$-invariante de $i(A)$ em $i(X)$ e $\left.\tilde{r}\right|_{W \cap i(X)}: W \cap i(X) \rightarrow i(A)$ é uma $G$-retração. Daí, $i^{-1}(W \cap i(X))=V \subset X$ e definimos $r: V \rightarrow A$ dada por $r(x)=i^{-1} \circ r \circ i(x)$.

Portanto os pares $(X, A) G$-ENR possuem a propriedade de existir uma vizinhança invariante $V \subset X$ de $A$ que se retrai equivariantemente em $A$. O próximo resultado é uma observação relacionada aos resultados de [23] que possibilitará trabalharmos com poliedros para criar resultados para variedades. É importante observar que não usaremos todo o conhecimento concebido nos resultados de [23], porque lá existem aspectos que não estão relacionados com o foco desse trabalho.

Proposição 2.3 : Sejam $G$ um grupo finito, $X$ uma $G$-variedade suave e $\left\{A_{i}\right\}_{i=1}^{n}$ uma coleção finita de $G$-subvariedades suaves e fechadas de $X$. Então, existe uma $G$-triangulação $K$ de $X$ e uma coleção finita de G-subcomplexos $\left\{L_{i}\right\}_{i=1}^{n}$ de $K$ tais que $L_{i}$ é uma $G$ triangularização de $A_{i}$, para $i=1, \ldots, n$.

Prova da Proposição 2.3: Primeiramente, tomemos $A$ e $B$ duas $G$-subvariedades suaves e fechadas de $X$. Pelo teorema 3.6 de [23] existem triangularizações equivariantes $\left.f_{1}:\left|K^{\prime}\right|,\left|L^{\prime}\right|\right) \rightarrow(X, A)$ e $f_{2}:\left(\left|R^{\prime}\right|,\left|Q^{\prime}\right|\right) \rightarrow(X, B)$. Em particular, temos que $f_{1}:\left|K^{\prime}\right| \rightarrow$ $X$ e $f_{2}:\left|R^{\prime}\right| \rightarrow X$ são duas $G$-triangularizações de $X$. Daí, segue do item 2 do Teorema da página 199 de [23] que existem $G$-subdivisões $K$ e $R$ de $K^{\prime}$ e $R^{\prime}$, respectivamente, tais que $f_{1}^{\prime}$ e $f_{2}^{\prime}$ são $G$-triangularizações suaves e $f_{1}^{\prime-1} \circ f_{2}^{\prime}:|R| \rightarrow|K|$ é um $G$-homeomorfismo simplicial.

Pelo corolário 3.3.5 (página 122) de [2] existem únicos subcomplexos $L$ de $K$ e $\bar{Q}$ de $R$ tais que $L$ e $\bar{Q}$ são subdivisões de $L^{\prime}$ e $Q^{\prime}$, respectivamente.

Da unicidade segue que $L$ e $\bar{Q}$ são $G$-subcomplexos de $K$ e $R$, respectivamente. De fato, $L^{\prime}$ é um $G$-subcomplexo de $K^{\prime}, L$ é o único subcomplexo de $K$ que subdivide $L^{\prime}$, então $L$ é invariante pela ação de $G$. O mesmo argumento vale para $\bar{Q}$ e $R$.

Como $f_{1}^{\prime-1} \circ f_{2}^{\prime}$ é um $G$-homeomorfismo simplicial (Teorema 3.7 de [23]) existe um $G$-subcomplexo $Q$ em $K$ tal que $f_{1}^{\prime-1} \circ f_{2}^{\prime}(\bar{Q})=Q\left(f_{1}^{\prime}(Q)=f_{1}^{\prime} \circ f_{1}^{\prime-1} \circ f_{2}^{\prime}(\bar{Q})=f_{2}^{\prime}(\bar{Q})=\right.$ 
$B)$. Daí, a $G$-triangulação $K$ de $X$ possui $G$-subcomplexos $L$ e $Q$ de $K$ que são $G$ triangularizações de $A$ e $B$, respectivamente.

Por indução, suponhamos que existe uma triangulação equivariante $K$ de $X$ e uma coleção finita de $G$-subcomplexos $\left\{L_{i}\right\}_{i=1}^{n-1}$ de $K$ tais que $L_{i}$ é uma $G$-triangularização de $A_{i}$, para $i=1, \ldots, n-1$ e seja $f_{2}:\left(R, Q_{n}\right) \rightarrow\left(X, A_{n}\right)$ uma $G$-triangulação de $\left(X, A_{n}\right)$.

Em particular, temos que $f_{1}:|K| \rightarrow X$ e $f_{2}:|R| \rightarrow X$ são duas $G$-triangularizações de $X$. Daí, pelo item 2 do teorema principal de [23] e pelo corolário 3.3.5 de [2] temos que existem subdivisões equivariantes $K^{\prime}$ e $R^{\prime}$ de $K$ e $R$, respectivamente, tais que $f_{1}^{\prime}$ e $f_{2}^{\prime}$ são $G$-triangularizações suaves, $f_{1}^{\prime-1} \circ f_{2}^{\prime}:\left|R^{\prime}\right| \rightarrow\left|K^{\prime}\right|$ é um $G$-homeomorfismo simplicial e existem únicos $G$-subcomplexos $L_{i}^{\prime}, i=1, \ldots, n-1$, de $K^{\prime}$ e $\overline{L_{n}^{\prime}}$ de $R^{\prime}$ tais que $L_{i}^{\prime}$ é uma subdivisão de $L_{i}, i=1 \ldots, n-1$, e $\overline{L_{n}^{\prime}}$ é uma subdivisão de $Q_{n}$.

Como $f_{1}^{\prime-1} \circ f_{2}^{\prime}$ é um $G$-homeomorfismo simplicial existe um $G$-subcomplexo $L_{n}^{\prime}$ em $K^{\prime}$ tal que $f_{1}^{\prime-1} \circ f_{2}^{\prime}\left(\overline{L_{n}^{\prime}}\right)=L_{n}^{\prime}\left(f_{1}^{\prime}\left(L_{n}^{\prime}\right)=f_{1}^{\prime} \circ f_{1}^{\prime-1} \circ f_{2}^{\prime}\left(\overline{L_{n}^{\prime}}\right)=f_{2}^{\prime}\left(\overline{L_{n}^{\prime}}\right)=A_{n}\right)$. Daí, a triangulação equivariante $K^{\prime}$ de $X$ é tal que existe uma coleção finita de $G$-subcomplexos $\left\{L_{i}^{\prime}\right\}_{i=1}^{n}$ de $K^{\prime}$ tais que $L_{i}^{\prime}$ é uma triangularização equivariante de $A_{i}$, para $i=1, \ldots, n$.

Na próxima proposição, cujas ideias foram retiradas de [20], usaremos $\operatorname{St}(A, K)=$ $\bigcup_{\substack{|\bar{t}| \cap A \neq \emptyset \\ t \in K}}|t|$ para um dado subconjunto $A$ de $|K|$, onde $K$ é um complexo simplicial. Faremos essa mudança de notação para que não ocorra ambiguidade com relação ao complexo simplicial em questão.

Esse resultado é a ponte que usaremos para conectar os subpoliedros aos conjuntos localmente contráteis. Assim, sob certas hipóteses, será possível realizar um conjunto localmente contrátil como o conjunto de pontos fixos de uma dada $G$-aplicação.

Proposição 2.4 Dados $G$ um grupo finito, $X$ um $G$-complexo simplicial compacto $e$ A um $G$-subconjunto de $X$ fechado. Então a St $(A, X)$ é uma vizinhança aberta e $G$ invariante de $A$ em $X$.

Além disso, se $X$ for a $G$-triangularização de uma $G$-variedade suave compacta com $\operatorname{dim}(X)>2$ e A for localmente contrátil e contornável em $X$ então existe uma subdivisão $X^{\prime}$ de $X$ na qual $\overline{S t\left(A, X^{\prime}\right)}$ é contornável em $X^{\prime}$.

Prova da Proposição 2.4: Definimos:

$$
S t(A, X)=\bigcup_{\substack{|\overline{\mid}| \cap A \neq \emptyset \\ t \in X}}|t| .
$$

Seja $\tilde{S} t(A, X)$ o subcomplexo de $X$ gerado por todos os simplexos $t$ de $X$ tais que $\overline{|t|} \cap A \neq \emptyset$, isto é, $\tilde{S} t(A, X)$ é o subcomplexo simplicial de $X$ que possui todas as faces dos simplexos $t$ de $X$ tais que $\overline{|t|} \cap A \neq \emptyset$. Segue que $\operatorname{Int}(|\tilde{S} t(A, X)|)=S t(A, X)$ (ou que $|\tilde{S} t(A, X)|=\overline{S t(A, X)})$ e assim $S t(A, X)$ é uma vizinhança aberta de $A$ em $X$. 
Para provar que $S t(A, X)$ é $G$-invariante seja $x$ um vértice de $\tilde{S} t(A, X)$ segue que $x \in \sigma$, onde $\overline{|\sigma|} \cap A \neq \emptyset$. Assim, existe $a \in \overline{|\sigma|} \cap A$. Dado $g \in G$ temos que $g x$ é um vértice de $g \sigma$ e $g a \in g \overline{|\sigma|} \cap A$. Portanto $g x \in \tilde{S} t(A, X)$ o que prova que $\tilde{S} t(A, X)$ é $G$-invariante, pois as $G$-órbitas dos vértices de $\tilde{S} t(A, X)$ ainda são vértices de $\tilde{S} t(A, X)$. Assim $\operatorname{St}(A, X)=\operatorname{Int}(|\tilde{S} t(A, X)|)$ também é $G$-invariante.

Se $A$ for localmente contrátil e contornável em $X$ então aplicando o teorema 2.2 de [20] em cada componente conexa de $X$ temos uma vizinhança $U$ de $A$ que é contornável em $X$. Daí, existe um $G$-refinamento $X^{\prime}$ de $X$ pequeno o suficiente para que $\overline{S t\left(A, X^{\prime}\right)} \subset U$.

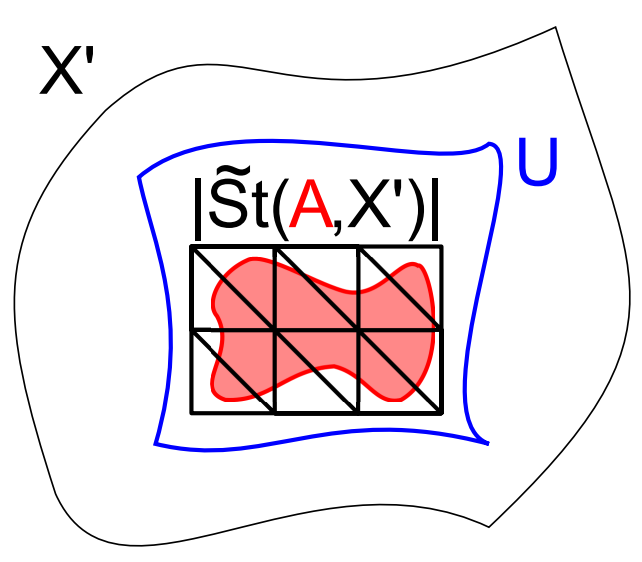

Figura 2.2: $\left|\tilde{S} t\left(A, X^{\prime}\right)\right|$

Agora, basta provar que $\overline{S t\left(A, X^{\prime}\right)}$ é contornável em $X^{\prime}$. Como $U$ e $A$ são contornáveis em $X^{\prime}$ resta verificar o que acontece com os caminhos $p: I \rightarrow X^{\prime}$ que começam em $U-\overline{S t\left(A, X^{\prime}\right)}$, terminam fora de $\overline{S t}\left(A, X^{\prime}\right)$ e passam por $\overline{S t\left(A, X^{\prime}\right)}$. Notemos que $p$ pode ser tomado fora de $A$, pois $p$ começa e termina fora de $A$ e $A$ é contornável em $X^{\prime}$.

Como $X^{\prime}$ não possui pontos de corte local e $\operatorname{dim}\left(X^{\prime}\right)>2$ podemos mover $p$ para $\partial\left(S t\left(A, X^{\prime}\right)\right)$, faremos isso usando o Corolário 3.3.11 de [2] da seguinte maneira:

Seja $\tilde{S} t\left(A, X^{\prime}\right)$ o subcomplexo de $X^{\prime}$ gerado por todos os simplexos $t$ de $X^{\prime}$ tais que $\overline{|t|} \cap A \neq \emptyset \mathrm{e}$ suponhamos que $p$ passa apenas pelos fechos das realizações geométricas dos simplexos $t_{1}, \ldots, t_{n} \in$ $\tilde{S} t\left(A, X^{\prime}\right)$. Já sabemos que $p$ não passa por $A$, assim cada um dos simplexos $t_{1}, \ldots, t_{n}$ possuem vértices em $\partial\left(\left|\tilde{S} t\left(A, X^{\prime}\right)\right|\right)$. Digamos que $\left\{v_{1}, \ldots, v_{l}\right\}$ são os vértices dos simplexos $t_{1}, \ldots t_{n}$ que estão em $\partial\left(\left|\tilde{S} t\left(A, X^{\prime}\right)\right|\right)$.

Sejam $K$ o subcomplexo de $X^{\prime}$ gerado por $t_{1}, \ldots, t_{n}$ (aqui não estou pedindo que $K$ seja invariante) e seja $L$ o subcomplexo de $X^{\prime}$ formado por simplexos cujos vértices estão em $\left\{v_{1}, \ldots, v_{l}\right\}$. Então aplicamos o Corolário 3.3.11 de [2] em $(K, L)$ e movemos $p$ para $\partial\left(\operatorname{St}^{\prime}\left(A, X^{\prime}\right)\right)$.

Agora, usaremos que $\overline{S t\left(A, X^{\prime}\right)} \subset U, X^{\prime}$ não possui pontos de corte local e que $\operatorname{dim}\left(X^{\prime}\right)>2$ para mover $p$ para longe de $S t\left(A, X^{\prime}\right)$. Temos que $p(I) \cap\left|\tilde{S} t\left(A, X^{\prime}\right)\right| \subset L$, sejam $s_{1}, \ldots, s_{d}$ simplexos maximais de $X^{\prime}$ tais que $\left|s_{i}\right| \cap\left(U-S t\left(A, X^{\prime}\right)\right) \neq \emptyset$ e alguma face de $s_{i}$ está em $L$. Seja $T$ o subcomplexo de $X^{\prime}$ gerado por $s_{1}, \ldots, s_{d}$. Aplicamos o Corolário 3.3.11 de [2] em $(T, L)$ e movemos $p$ para o interior de $T$. Assim $p$ é homotópico a uma caminho $p^{\prime}$ tal que $p^{\prime}(I) \cap \overline{S t\left(A, X^{\prime}\right)}=\emptyset$.

Nem sempre a união de dois subespaços contornáveis $A$ e $Y$ em um espaço $X$, é um subespaço contornável, pois essa propriedade não decorre do fato de $A$ e $Y$ serem separadamente contornáveis em $X$. De fato, tomando $X=S^{2}, A=\left\{(x, y, z) \in S^{2} ; z>\right.$ 0 ou $\left.x={ }_{-}^{+} 1\right\}$ e $Y=\left\{(x, y, z) \in S^{2} ; z<0\right.$ ou $\left.x={ }_{-}^{+} 1\right\}$ criamos um exemplo onde $A$ e $Y$ 
são separadamente contornável em $X$ mas $A \cup Y$ não é contornável em $X$.

As ideias do próximo resultados são encontradas em [11].

Proposição 2.5 : Sejam $X$ a triangularização de uma variedade conexa e compacta tal que $\operatorname{dim}(X)>2,\left\{Y_{i}\right\}_{i=1}^{n}$ uma coleção finita Figura 2.3: A e $Y$ conde subpoliedros fechados de $X$ tais que $\operatorname{dim}\left(Y_{i}\right)+1<\operatorname{dim}(X)$, tornáveis $Y=\bigcup_{i=1}^{n} Y_{i}$ e A um subconjunto não vazio e contornável em $X$. Então $A \cup Y$ é contornável em $X$.

Prova da Proposição 2.5: Como o poliedro $X$ é conexo e compacto temos que seu grupo fundamental é finitamente gerado para qualquer ponto base. Tomemos um ponto base $a \in X-(A \cup Y)$ e os geradores $p_{1}, \ldots, p_{k}: I \rightarrow X$ de $\pi_{1}(X, a)$.

Como $A$ é contornável em $X$ podemos supor que $p_{1}, \ldots, p_{k}: I \rightarrow X-A$. Seja $t_{0} \in I$ o primeiro ponto tal que $p_{i}\left(t_{0}\right) \in p_{i}(I) \cap Y$ e $|\sigma|$ a realização geométrica do simplexo maximal de $X$ que contém $p_{i}\left(t_{0}\right)$, digamos que $|\sigma|$ é $n$-dimensional. Segue que $Y$ intercepta $|\sigma|$ em uma face $\omega_{1}$, que é no máximo $(n-2)$-dimensional, pois $\operatorname{dim}\left(Y_{i}\right)+1<\operatorname{dim}(X)$. Daí, $\omega_{1}$ está contida em uma face $\omega_{0}$ de $\sigma$ que é no máximo $(n-1)$-dimensional.

Logo, existe um ponto em $\omega_{0}$ pelo qual podemos desviar $p_{i}$ em uma vizinhança de $t_{0}$. Como $I$ é compacto $p_{i}$ é homotópico a um caminho $p_{i}^{\prime}: I \rightarrow X-(A \cup Y)$. Portanto, o homomorfismo induzido pela inclusão $i_{*}: \pi_{1}(X-(A \cup Y)) \rightarrow \pi_{1}(X)$ é sobrejetor e o resultado segue do Teorema 5.2 de [11]. 


\subsection{Procedimentos em $G$-Classes de Pontos Fixos}

Para realizar um conjunto como o conjunto de pontos fixos de uma dada $G$-classe de homotopia será necessário excluir todos os possíveis pontos fixos, de um dado representante dessa $G$-classe de homotopia, que estejam fora do conjunto em questão. O objetivo dessa seção é criar procedimentos que nos permitam excluir $G$-órbitas de pontos fixos. As construções que fizemos nessa subseção foram baseadas em [25].

Procedimento 2.6 : Sejam $G$ um grupo finito, $X$ uma $G$-variedade suave, compacta e $\operatorname{dim}(X)>2,\left\{Y_{i}\right\}_{i=1}^{n}$ uma coleção finita de G-subvariedades fechadas de $X$ tal que $\operatorname{dim}\left(Y_{i}\right)+1<\operatorname{dim}(X)$ e $G$ age livremente em $X-Y \operatorname{com} Y=\bigcup_{i=1}^{n} Y_{i}$.

Sejam A um G-subconjunto de $X$, localmente contrátil, fechado, não vazio e tal que $A$ é contornável em $X$ e $f: X \rightarrow X$ uma $G$-aplicação tal que $A \subset F i x(f), f$ não possui pontos fixos em $Y-A$ e $f$ possui uma quantidade finita de pontos fixos em $X-(A \cup Y)$.

Sejam $x_{0}$ e $x_{1}$ dois pontos fixos de $f G$-Nielsen equivalentes e em órbitas diferentes tais que $x_{0}, x_{1} \in X-(A \cup Y)$ e $q: I \rightarrow X$ um caminho de $q(0)=x_{0}$ até $q(1)=x_{1}$ tal que $f \circ q$ é homotópico a $q$ (relativo aos extremos). Então q é homotópico, relativo aos extremos, a um PL-caminho normal $p: I \rightarrow X$ (relativo a uma $G$-triangularização de $X$ ) tal que:

1. $p(I) \cap F i x(f)=\left\{x_{0}, x_{1}\right\}$ e $p(I) \cap(A \cup Y)=\emptyset$;

2. $p(I) \cap g p(I)=\emptyset$, para todo $g \in G-\{e\}$;

3. $f \circ p$ é homotópico a $p$, relativo aos extremos.

Prova do Procedimento 2.6: Fixemos uma $G$-triangularização de $X$ tal que $Y_{i}$, para $i=1, \ldots, n$, seja um $G$-subcomplexo, isso é possível pela Proposição 2.3. Pela proposição 2.4 essa triangularização pode ser tomada pequena o suficiente para que $S t(A)$ seja contornável em $X$.

Como $f$ possui uma quantidade finita de pontos fixos em $X-(A \cup Y)$ e $x_{i} \in X-$ $(A \cup Y)$, para $i=1,2$, podemos dizer que $f$ não tem pontos fixos em $S t(A)-A$ e que $x_{i} \in X-(S t(A) \cup Y)$ pois a triangularização é suficientemente pequena. Daí, basta usar que $(S t(A) \cup Y)$ é contornável em $X$ para encontrar um caminho $p_{1}: I \rightarrow X$, $p_{1}(I) \subset X-(S t(A) \cup Y)$, homotópico a $q$ relativo aos extremos.

Agora, aplicamos o Lema 3.1 de [8] para encontrar um PL-caminho normal $p_{2}: I \rightarrow X$ homotópico a $p_{1}$. O Lema 3.1 de [8] usa uma $\epsilon$-homotopia, para algum $\epsilon>0$, por isso podemos supor que sempre que $p_{2}$ passa de um simplexo maximal para outro essa transferência se passa em um simplexo de dimensão maior ou igual a 2.

Também podemos supor que $p_{2}(I) \cap F i x(f)=\left\{x_{0}, x_{1}\right\}$, pois $F i x(f)-(A \cup Y)$ é finito e $\operatorname{dim}(X)>2$. Assim, $p_{2}$ possui as seguintes propriedades:

1. $p_{2}(I) \cap F i x(f)=\left\{x_{0}, x_{1}\right\}$ e $p_{2}(I) \cap(S t(A) \cup Y)=\emptyset$;

2. $f \circ p_{2}$ é homotópico a $p_{2}$ relativo aos extremos. 
Falta mostrar que $p_{2}$ é homotópico a um caminho $p: I \rightarrow X$, relativo aos extremos, que possui as mesmas propriedades de $p_{2}$, anteriormente citadas, e com a propriedade adicional $p(I) \cap g p(I)=\emptyset$, para todo $g \in G-\{e\}$. O caminho $p$ será construído passo a passo.

Sejam $\left\{t_{0}, t_{1}, \ldots, t_{n+1}\right\} \subset I$ e $\left\{\sigma_{j}\right\}_{j=0}^{n}$ uma coleção de simplexos maximais de $X$ tais que:

1. $\left.p_{2}(] t_{j}, t_{j+1}[)=\right] p_{2}\left(t_{j}\right), p_{2}\left(t_{j+1}\right)[\in|\sigma j|$, para $j=0,1, \ldots, n$;

2. $p_{2}\left(t_{j}\right) \in\left|\sigma_{j-1} \cap \sigma_{j}\right|$, onde $\sigma_{j-1} \cap \sigma_{j}$ é um simplexo de $X$ de dimensão maior ou igual a 2 , para $j=1, \ldots, n$;

3. $t_{0}=0, t_{n+1}=1, p_{2}(0) \subset\left|\sigma_{0}\right|$ e $p_{2}(1) \subset\left|\sigma_{n}\right|$ quando $x_{1} \in X-(A \cup Y)$ ou $p_{2}(1) \subset \partial\left(\left|\sigma_{n}\right|\right) \cap A$ quando $x_{1} \in \partial(A)$.

O primeiro passo é $t \in\left[0, t_{1}\right]$. Se $j \in\{0,1, \ldots, n\}$ e $g \in G$ são tais que $p_{2}\left(\left[0, t_{1}\right]\right) \subset$ $g p_{2}\left(\left[t_{j}, t_{j+1}\right]\right)$. Então, $g^{-1} p_{2}(0) \in p_{2}\left(\left[t_{j}, t_{j+1}\right]\right) \cap F i x(f)$, mas $p_{2}(I) \cap F i x(f)=\left\{x_{0}, x_{1}\right\}$, $\operatorname{assim} j=0$ e $g=e$.

Agora trabalharemos em $\left[0, t_{n}\right]$. Seja $l \in\{0,1, \ldots, n-1\}$ o primeiro índice no qual existem $j \in\{0,1, \ldots, n\}$ e $g \in G$ tais que:

$$
p_{2}\left(\left[t_{l}, t_{l+1}\right]\right) \cap g p_{2}\left(\left[t_{j}, t_{j+1}\right]\right) \neq \emptyset .
$$

$p_{2}$ é um PL-caminho normal, daí, temos apenas as seguintes possibilidades de interseção entre $p_{2}\left(\left[t_{l}, t_{l+1}\right]\right)$ e $g p_{2}\left(\left[t_{j}, t_{j+1}\right]\right)$ :

1. existe $t \in] t_{l}, t_{l+1}\left[\right.$ tal que $p_{2}\left(\left[t_{l}, t_{l+1}\right]\right) \cap g p_{2}\left(\left[t_{j}, t_{j+1}\right]\right)=\left\{p_{2}(t)\right\}$;

2. $p_{2}\left(\left[t_{l}, t_{l+1}\right]\right) \cap g p_{2}\left(\left[t_{j}, t_{j+1}\right]\right)=\left\{p_{2}\left(t_{l+1}\right)\right\}$.

Estas situações estão ilustradas na figura 2.4, o primeiro triangulo da esquerda representa a primeira situação e os dois outros representam a segunda situação levando em conta o que pode ocorrer logo após tal interseção.
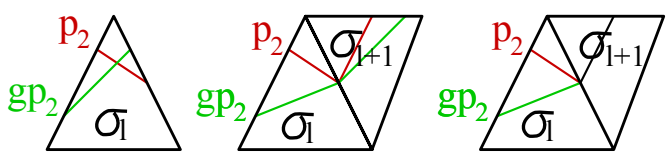

Figura 2.4: Interseções

Para ambos os casos vamos tomar um ponto $a \in\left|\sigma_{l} \cap \sigma_{l+1}\right|$ tal que:

(i) (]$\left.p_{2}\left(t_{l}\right), a\right] \cup\left[a, p_{2}\left(t_{l+1}\right)[) \cap\left(g p_{2}(I)\right)=\emptyset\right.$, para todo $g \in G$;

(ii) (]$\left.p_{2}\left(t_{l}\right), a\right] \cup\left[a, p_{2}\left(t_{l+1}\right)[) \cap(A \cup Y \cup F i x(f))=\emptyset\right.$; 
(iii) (]$\left.p_{2}\left(t_{l}\right), a\right] \cup\left[a, p_{2}\left(t_{l+1}\right)[) \cap g(] p_{2}\left(t_{l}\right), a\right] \cup\left[a, p_{2}\left(t_{l+1}\right)[)=\emptyset\right.$, para todo $g \in G-\{e\}$.

O elemento a existe porque $\operatorname{dim}(X) \geq 3, \operatorname{dim}\left(\sigma_{l} \cap \sigma_{l+1}\right) \geq 2$,

$$
\left(\bigcup_{g \in G} g p_{2}(I)\right) \cup\left(\bigcup_{g \in G} g(] p_{2}\left(t_{l}\right), a\right] \cup\left[a, p_{2}\left(t_{l+1}\right)[)\right)
$$

é um conjunto finito de segmentos de reta e

$$
p_{2}(t) \notin(A \cup Y \cup F i x(f)) \text { para todo } t \in\left[t_{l}, t_{l+2}[.\right.
$$

Essa situação está ilustrada na figura 2.5. Daí, o PL-caminho normal dado por:

$$
\begin{aligned}
p_{2}^{\prime}: I & \rightarrow \\
t & \mapsto \begin{cases}p_{2}(t) & \text { para } t \in\left[0, t_{l}\right] \cup\left[t_{l+2}, 1\right] \\
\left(\frac{t_{l+1}-t}{t_{l+1}-t_{l}}\right) p_{2}\left(t_{l}\right)+\left(\frac{t-t_{l}}{t_{l+1}-t_{l}}\right) a & \text { para } t \in\left[t_{l}, t_{l+1}\right] \\
\left(\frac{t_{l+2}-t}{t_{l+2}-t_{l+1}}\right) a+\left(\frac{t-t_{l+1}}{t_{l+2}-t_{l+1}}\right) p_{2}\left(t_{l+2}\right) & \text { para } t \in\left[t_{l+1}, t_{l+2}\right]\end{cases}
\end{aligned}
$$

é homotópico a $p_{2}$, relativo aos extremos, por uma homotopia linear, uma vez que, estamos alterando $p_{2}$ apenas em $\left|\sigma_{l} \cup \sigma_{l+1}\right|$ substituindo $p_{2}\left(\left[t_{l}, t_{l+2}\right]\right)$ por $\left[p_{2}\left(t_{l}\right), a\right] \cup\left[a, p_{2}\left(t_{l+2}\right)\right] \mathrm{em}$ $p_{2}$.
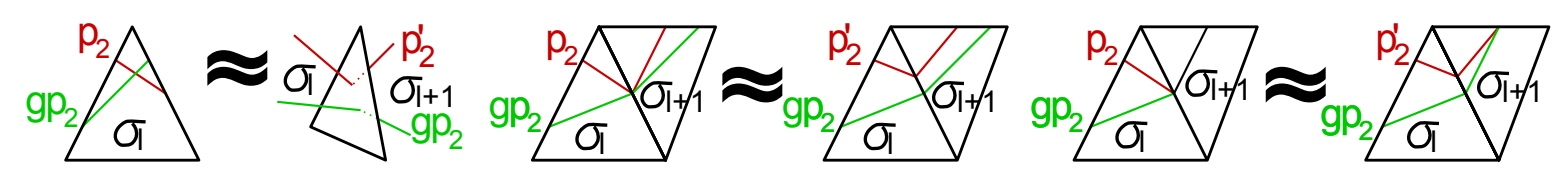

Figura 2.5: Homotopia

Assim, por um processo indutivo concluímos que existe um PL-caminho normal $p_{3}$ homotópico a $p_{2}$, relativo aos extremos, tal que:

1. $p_{3}(I) \cap F i x(f)=\left\{x_{0}, x_{1}\right\}, p_{3}\left(\left[0,1[) \cap A=\emptyset\right.\right.$ e $p_{3}([0,1[) \cap Y=\emptyset ;$

2. $p_{3}\left(\left[0, t_{n}\right]\right) \cap g p_{3}\left(\left[0, t_{n}\right]\right)=\emptyset$, para todo $g \in G-\{e\}$;

3. $f \circ p_{3}$ é homotópico a $p_{3}$ (relativo aos extremos).

Agora só falta trabalhar em $\left[t_{n}, 1\right]$. Se existirem $j \in\{0,1, \ldots, n\}$ e $g \in G$ tais que:

$$
p_{3}\left(\left[t_{n}, 1\right]\right) \cap g p_{3}\left(\left[t_{j}, t_{j+1}\right]\right) \neq \emptyset .
$$

Observamos que se $\left\{p_{3}(1)\right\} \in p_{3}\left(\left[t_{n}, 1\right]\right) \cap g p_{3}\left(\left[t_{j}, t_{j+1}\right]\right)$, para algum $j$ e algum $g$, então $g^{-1} p_{3}(1) \in p_{3}\left(\left[t_{j}, t_{j+1}\right]\right) \cap F i x(f)$, mas $p_{3}(I) \cap F i x(f)=\left\{x_{0}, x_{1}\right\}$, assim $j=n$ e $g=e$. Segue que a única possibilidade de interseção ocorre se existir $t \in] t_{n}, 1[$ tal que

$$
p_{3}\left(\left[t_{n}, 1\right]\right) \cap g p_{3}\left(\left[t_{j}, t_{j+1}\right]\right)=\left\{p_{3}(t)\right\} .
$$


Mas, se esse for o caso basta aplicar o procedimento anterior para $i=n-1$.

Observamos que nem todas as propriedades descritas no procedimento anterior se perdem quando $x_{0}$ e $x_{1}$ não são pontos fixos de $f$. O próximo resultado explicita com maior precisão esse comentário.

Corolário 2.7 : Sejam $G$ um grupo finito, $X$ uma $G$-variedade suave, compacta com $\operatorname{dim}(X)>2,\left\{Y_{i}\right\}_{i=1}^{n}$ uma coleção finita de $G$-subvariedades fechadas de $X$ tal que $\operatorname{dim}\left(Y_{i}\right)+1<\operatorname{dim}(X)$ e $G$ age livremente em $X-Y$ com $Y=\bigcup_{i=1}^{n} Y_{i}$.

Sejam A um $G$-subconjunto de $X$, localmente contrátil, fechado, não vazio e tal que $A$ é contornável em $X$ e $f: X \rightarrow X$ uma $G$-aplicação tal que $A \subset F i x(f), f$ não possui pontos fixos em $Y-A$ e $f$ possui uma quantidade finita de pontos fixos em $X-(A \cup Y)$.

Sejam $x_{0}$ e $x_{1}$ dois pontos de $X$ situados em $X-(A \cup Y)$ e conectados por uma caminho $q: I \rightarrow X\left(q(0)=x_{0}\right.$ e $\left.q(1)=x_{1}\right)$. Então $q$ é homotópico, relativo aos extremos, a um PL-caminho normal $p: I \rightarrow X$ (relativa a uma $G$-triangularização de $X$ ) tal que:

1. $p(] 0,1[) \cap F i x(f)=\emptyset$ e $p(I) \cap(A \cup Y)=\emptyset$;

2. $p(I) \cap g p(I)=\emptyset$, para todo $g \in G-\{e\}$;

Prova do Corolário 2.7: Assim como fixemos no procedimento anterior, fixemos uma $G$-triangularização de $X$ tal que $Y_{i}$, para $i=1, \ldots, n$, seja um $G$-subcomplexo e pequena o suficiente para que $S t(A)$ seja contornável em $X$. Também podemos dizer que $f$ não tem pontos fixos em $S t(A)-A$ e que $x_{i} \in X-(S t(A) \cup Y)$. Daí, basta usar que $(S t(A) \cup Y)$ é contornável em $X$ para encontrar um caminho $p_{1}: I \rightarrow X$, $p_{1}(I) \subset X-(S t(A) \cup Y)$, homotópico a $q$ relativo aos extremos.

Depois, aplicamos o Lema 3.1 de [8] para encontrar um PL-caminho normal $p_{2}: I \rightarrow X$ homotópico a $p_{1}$ tal que $p_{2}(] 0,1[) \cap F i x(f)=\emptyset$. Por fim, basta aplicar, passo a passo, o processo indutivo descrito no final do Procedimento 2.6 para encontrar o caminho $p$ : $I \rightarrow X$ homotópico a $q$, relativo aos extremos, que possui as propriedades desejadas.

As ideias dos próximos procedimentos é transformar a $G$-aplicação $f$ em uma $G$ aplicação proximal próximo ao caminho que foi criado no procedimento 2.6 para depois aplicar o lema VIII.C.3 de [1].

Procedimento 2.8 : Sejam $G$ um grupo finito, $X$ uma $G$-variedade suave, compacta $e \operatorname{dim}(X)>2,\left\{Y_{i}\right\}_{i=1}^{n}$ uma coleção finita de G-subvariedades fechadas de $X$ tal que $\operatorname{dim}\left(Y_{i}\right)+1<\operatorname{dim}(X)$ e $G$ age livremente fora de $Y=\bigcup_{i=1}^{n} Y_{i}$.

Sejam $A$ um $G$-subconjunto de $X$, localmente contrátil, fechado, não vazio e tal que $A$ é contornável em $X$ e $f: X \rightarrow X$ uma $G$-aplicação tal que $A \subset F i x(f), f$ não possui pontos fixos em $Y-A$ e $f$ possui uma quantidade finita de pontos fixos em $X-(A \cup Y)$. 
Sejam $x_{0}$ e $x_{1}$ dois pontos fixos de $f G$-Nielsen equivalentes e em órbitas diferentes tais que $x_{0} \in X-(A \cup Y)$ e $x_{1} \in X-(A \cup Y)$ ou $x_{1} \in \partial(A)$, onde $\partial(A)$ é o bordo de A em $X$ e $q: I \rightarrow X$ um caminho de $q(0)=x_{0}$ até $q(1)=x_{1}$ tal que $f \circ q$ é homotópico a $q$ relativo aos extremos. Então $f$ é $G$-homotópica relativo a $(A \cup Y)$ a uma aplicação $h: X \rightarrow X$ tal que Fix $(h)=F i x(f)-G\left\{x_{0}\right\}$.

Prova do Procedimento 2.8: Fixemos uma $G$-triangularização de $X$ tal que $Y_{i}$, para $i=1, \ldots, n$, seja um $G$-subcomplexo. Pela proposição 2.4 essa triangularização pode ser tomada pequena o suficiente para que $S t(A)$ seja contornável em $X$ e como $f$ possui uma quantidade finita de pontos fixos em $X-(A \cup Y)$ podemos dizer que $f$ não tenha pontos fixos em $S t(A)-A$.

Notemos que os pontos fixos de $f$ que estão fora de $A \cup Y$ são isolados. Vamos considerar os casos. No primeiro tomamos $x_{0}$ um ponto fixo isolado tal que o índice de $x_{0}$ é 0. Pelo Teorema VIII.B.4 de [1], obtemos uma homotopia $D^{\prime}$ em uma vizinhança $W$ de $x_{0}$ relativa a $\partial(W)$ que pode ser tomada de modo que $W \cap g W=\emptyset$ para todo $g \in G$. Daí, para cada $(x, t) \in g W \times I$, com $g \in G$, definimos $D(x, t)=g D^{\prime}\left(g^{-1} x, t\right)$ que é uma homotopia em $g W$ relativa a $\partial(g W)$, para todo $g \in G$. Assim, obtemos a extensão equivariante da homotopia. Nesse caso, diremos que estendemos a homotopia obtida por equivariância.

No segundo caso, tomamos $x_{0}$ um ponto fixo isolado com índice diferente de $0, x_{1} \notin$ $\partial(A)$ e $x_{0}$ e $x_{1}$ são $G$-Nielsen equivalentes. Com o procedimento 2.6 encontramos um PL-caminho normal $s: I \rightarrow X$ ligando $x_{0}=s(0)$ à $x_{1}=s(1)$ e tal que:

1. $s(I) \cap F i x(f)=\left\{x_{0}, x_{1}\right\}$ e $s(I) \cap(A \cup Y)=\emptyset$;

2. $s(I) \cap g s(I)=\emptyset$, para todo $g \in G-\{e\}$;

3. $f \circ s$ é homotópico a $s$ (relativo aos extremos).

Queremos aplicar o lema VIII.C.3 de [1] para colapsar a $G$-órbita de $x_{0}$ a $G$-órbita de $x_{1}$. Contudo, temos que garantir que $f$ é $G$-homotópica a uma aplicação que é proximal em algum caminho que conecte a $G$-órbita de $x_{0}$ à $G$-órbita de $x_{1}$. Seja $\epsilon>0$ um número suficientemente pequeno, e definimos o caminho:

$$
\begin{aligned}
& s_{\epsilon}: I \rightarrow \quad X \\
& t \mapsto s(t-\epsilon \sin (t \pi)) \text {. }
\end{aligned}
$$

Escolhemos $\epsilon>0$ de modo que $s_{\epsilon} \circ s^{-1}: s(I) \rightarrow X$ seja uma aplicação proximal. Temos que $s_{\epsilon}$ e $f \circ s$ são caminhos especiais com relação a $s$ e são homotópicos entre si. Pelo Lema 5.1 de [8] segue que $s_{\epsilon}$ e $f \circ s$ são especialmente homotópicos. Portanto as aplicações $\left.f\right|_{s(I)}, s_{\epsilon} \circ s^{-1}: s(I) \rightarrow X$ são especialmente homotópicas.

Podemos estender a homotopia $\overline{{ }_{1} H}: s(I) \times I \rightarrow X$ entre $\left.f\right|_{s(I)}$ e $s_{\epsilon} \circ s^{-1}$ para uma $G$-aplicação ${ }_{1} H^{\prime}:\left((A \cup Y) \cup\left(\bigcup_{g \in G} g s(I)\right)\right) \times I \rightarrow X$ tal que:

$$
{ }_{1} H^{\prime}(x, t)= \begin{cases}f(x) & \text { se } t=0 \text { ou } x \in(A \cup Y) \\ g\left(\overline{{ }_{1} H}\left(g^{-1} x, t\right)\right) & \text { se } x \in \text { gs }(I) \text { para algum } g \in G .\end{cases}
$$


Quando $x_{1} \in \partial(A)$ temos que ${ }_{1} H^{\prime}$ é contínua em $g x_{1}$, para todo $g \in G$, porque ${ }_{1} H\left(x_{1}, t\right)=x_{1}=f\left(x_{1}\right)$ para todo $t \in I$.

No apêndice A Retração Local criamos duas retrações bem particulares que usaremos nesse procedimento para estender a homotopia acima. Lembremos que $s$ é um PLcaminho normal, daí, se $x_{1} \in X-(A \cup Y)$ então existe uma $\delta$-vizinhança fechada $U(s(I))$ de $s_{\epsilon} \circ s^{-1}(s(I)), \delta>0$, e existe um homeomorfismo $h: W \subset \mathbb{R}^{n} \rightarrow U(s(I))$, onde $W$ é uma $\delta$-vizinhança fechada de $J=\left\{(x, 0, \ldots, 0) \in \mathbb{R}^{n} ; 0 \leq x \leq\right.$ $1\}$ e $h$ é tal que $h(x, 0, \ldots, 0)=s(x)$ para todo $x \in[0,1]$.

Definimos a retração:

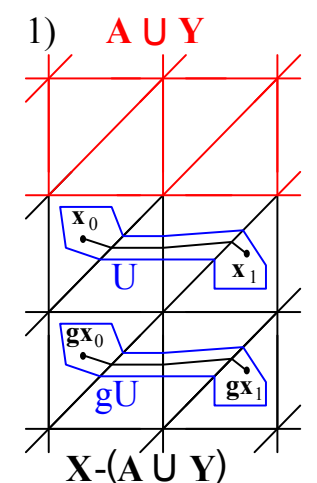

Figura 2.6: $U(s(I))$

$$
R^{\prime}: U(s(I)) \times I \rightarrow(U(s(I)) \times\{0\}) \cup(s(I) \times I)
$$

dada por:

$$
R^{\prime}(x, t)=\left(h \circ \pi_{1}\left(R_{1}\left(h^{-1}(x), t\right)\right), \pi_{2}\left(R_{1}\left(h^{-1}(x), t\right)\right)\right)
$$

onde $R_{1}: W \times I \rightarrow(W \times\{0\}) \cup(J \times I)$ é uma das retrações do apêndice Retração Local, $\pi_{1}$ e $\pi_{2}$ são as projeções e $R^{\prime}$ é tal que $R^{\prime}(x, t)=(x, 0)$ se $(x, t) \in \partial(U(s(I))) \times I$ e $R_{1}^{\prime}(x, t)=(x, t)$ se $(x, t) \in(U(s(I)) \times\{0\}) \cup(s(I) \times I)$. Podemos tomar $\delta$ tão pequeno que:

- $F i x(f) \cap U(s(I))=\left\{x_{0}, x_{1}\right\}$;

- $U(s(I)) \cap g U(s(I))=\emptyset$, para $g \in G-\{e\}$;

- $(A \cup Y) \cap g U(s(I))=\emptyset$, para $g \in G-\{e\}$.

Daí, podemos estender $R^{\prime}$ para uma $G$-aplicação $R$ definida em $\left(\bigcup_{g \in G} g \bar{U}(s(I))\right) \times I$ por $R(x, t)=g R^{\prime}\left(g^{-1} x, t\right)$, onde $x \in g \bar{U}(s(I))$ e $g \in G$. Com $R$ estendemos a $G$-aplicação ${ }_{1} H^{\prime}:\left((A \cup Y) \cup\left(\bigcup_{g \in G} g s(I)\right)\right) \times I \rightarrow X$ para uma $G$-homotopia ${ }_{1} H: X \times I \rightarrow X$ da seguinte forma:

$$
{ }_{1} H(x, t)= \begin{cases}f(x) & \text { se } t=0 \text { ou } x \notin \cup_{g \in G} g \bar{U}(s(I)) \\ { }_{1} H^{\prime} \circ R(x, t) & \text { se } x \in g U(s(I)) \text { para algum } g \in G\end{cases}
$$

${ }_{1} H$ é contínua porque quando $x \in \partial(U(s(I)))$ temos que $R(x, t)=(x, 0), \log { }_{1} H^{\prime} \circ$ $R(x, t)={ }_{1} H^{\prime}(x, 0)=f(x)$. Daí, estendemos ${ }_{1} H^{\prime}$ para uma $G$-homotopia ${ }_{1} H: X \times I \rightarrow X$ tal que $F i x(f)=F i x\left({ }_{1} H_{t}\right)$ para todo $t \in I$ e $\left.{ }_{1} H(\bullet, 1)\right|_{s(I)}$ é uma aplicação proximal.

Dessa forma, podemos usar o Lema VIII.C.3 de [1], para mover $g x_{0}$ ao longo de $g s_{\epsilon}(I)$, com $g \in G$, como segue: seja $U$ uma vizinhança de $s_{\epsilon}$ tal que $\bar{U} \cap((A \cup Y) \cup g \bar{U})=\emptyset$, para $g \in G-\{e\}$. Com o lema VIII.C.3 de [1] movemos $x_{0}$ ao longo de $s_{\epsilon}(I)$ por homotopias que ocorrem dentro de $U$ e colapsamos a órbita de $x_{0}$ na órbita de $x_{1}$. 
Lembrando que cada vez que usamos o lema obtemos uma homotopia $D^{\prime}$ em uma vizinhança $W \subset U$ que estendemos por equivariância. Assim, movemos $g x_{0}$ ao longo de $g s_{\epsilon}(I)$, com $g \in G$.

Para o terceiro, e último, caso $x_{1} \in \partial(A)$ fixemos um ponto $x_{2} \in X-(A \cup Y)$ suficientemente próximo de $x_{1}$ para que:

1. o segmento ] $x_{1}, x_{2}$ ] esteja fora de $A \cup Y$ e totalmente contido no interior da realização geométrica de um simplexo maximal, digamos $\sigma$;

2. $\left.\left.\left.\left.(g] x_{1}, x_{2}\right]\right) \cap\right] x_{1}, x_{2}\right]=\emptyset$, para todo $g \in G-\{e\}$;

3. $\left.\left.(g] x_{1}, x_{2}\right]\right) \cap F i x(f)=\emptyset$, para todo $g \in G$.

Daí, a concatenação de $q$ com $\left[x_{1}, x_{2}\right]$ é um caminho que começa e termina fora de

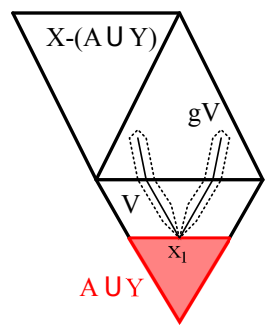

Figura 2.7: Lema VIII.C.3 $A \cup Y$. A proposição 2.5 aplicada em cada componente conexa de $X$ garante que $A \cup Y$ é contornável em $X$. Daí, encontramos um PL-caminho normal $p_{1}^{\prime}: I \rightarrow X$, cujas propriedades estão descritas no Corolário 2.7, homotópico a $q *\left[x_{1}, x_{2}\right]$ e tal que $p_{1}^{\prime}(I) \subset X-(A \cup Y)$.

Consequentemente, $p_{1}=p_{1}^{\prime} *\left[x_{2}, x_{1}\right]$ é homotópico a $q$ relativo aos extremos. Observemos que $p_{1}(I) \cap A=\left\{x_{1}\right\}$ e que $p_{1}([0,1[) \cap Y=\emptyset$.

Assim, podemos usar a mesma construção feita no caso anterior (o segundo caso) para mover a $G$-órbita de $x_{0}$ até a $G$-órbita de $x_{2}$.

É bom observar que no caso anterior usamos uma das retrações descritas no apêndice Retração Local e que nesse terceiro caso usamos a outra. Desse modo, criamos uma $G$ aplicação $k: X \rightarrow X$ equivariantemente homotópica a $f$ com $g x_{2} \in g|\sigma|$ pontos fixos isolados de $k$, para $g \in G, g x_{1} \in g|\sigma| \cap A$ pontos fixos de $k$, para $g \in G$ e $\left.k\right|_{\left[x_{2}, x_{1}[\right.}$ é uma aplicação proximal.

Seja $k: X \rightarrow X$ a $G$-aplicação $G$-homotópica a $f \operatorname{com} g x_{2} \in g|\sigma|$ e $g x_{1} \in g \mid \overline{|\sigma|} \cap A$ pontos fixos isolados de $k$ e $\left.k\right|_{\left[x_{2}, x_{1}[\right.}$ é uma aplicação proximal.

Tomemos uma vizinhança aberta $V$ de $\left[x_{2}, x_{1}[\right.$ em $|\sigma|$ convexa com relação a estrutura simplicial de $\sigma$ tal que:

1. $\partial V \cap(A \cup Y)=\left\{x_{1}\right\}$ e $(\bar{V} \cap g \bar{V})-\left\{x_{1}\right\}=\emptyset$ para todo $g \in G$;

2. $\bar{V} \subset \overline{|\sigma|}$ e $\bar{V} \cap \partial|\sigma|=\left\{x_{1}\right\}$

3. $k(\bar{V}) \subset \overline{|\sigma|}$, pois $k$ é uma aplicação proximal em $\left[x_{2}, x_{1}[\right.$. 
Notemos que todo ponto $x \in \bar{V}$ pode ser visto como um par da forma:

$x=(b(x), r(x)) \in \partial V \times I$, onde $x=r(x) b(x)+(1-r(x)) x_{1}$.

Como $\bar{V} \cap \overline{g V}=\emptyset$ para todo $g \in G-\{e\}$ definimos a homotopia linear $H: X \times I \rightarrow X$ dada por $k(x)$ se $x \in X-\bigcup_{g \in G} g \bar{V}$ e dada por:

$(1-t) k(x)+\operatorname{tg}\left(r\left(g^{-1} x\right) k \circ b\left(g^{-1} x\right)+\left(1-r\left(g^{-1} x\right)\right) x_{1}\right)$,

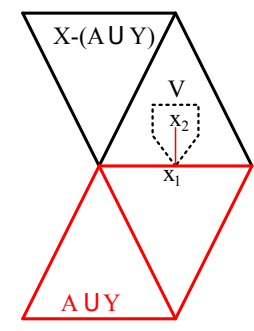

Figura 2.8: Vizinhança $V$

se $x \in \bigcup_{g \in G} g \bar{V}$. Essa homotopia é contínua porque se $x \in \partial(g V)$, para algum $g \in G$, então $g b\left(g^{-1} x\right)=x$ e $r\left(g^{-1} x\right)=1$, dessa forma:

$$
\begin{aligned}
(1-t) k(x)+\operatorname{tg}\left(r\left(g^{-1} x\right) k \circ b\left(g^{-1} x\right)+\left(1-r\left(g^{-1} x\right)\right) x_{1}\right) & = \\
(1-t) k(x)+t g\left(g^{-1} k(x)+(1-1) x_{1}\right) & =(1-t) k(x)+t k(x) \\
& =k(x) .
\end{aligned}
$$

Portanto, $f$ é $G$-homotópica relativo a $(A \cup Y)$ a uma $G$-aplicação $h: X \rightarrow X$ tal que $F i x(h)=F i x(f)-G\left\{x_{0}\right\}$.

Um ponto importante que se observa na demonstração dos procedimentos 2.6 e 2.8 é que esses resultados continuam válidos quando substituímos $A$ por um $G$-subpoliedro fechado de $X$.

Corolário 2.9 : Sejam $G$ um grupo finito, $X$ uma G-variedade suave, compacta tal que $\operatorname{dim}(X)>2,\left\{Y_{i}\right\}_{i=1}^{n}$ uma coleção finita de $G$-subvariedades fechadas de $X$ tal que $\operatorname{dim}\left(Y_{i}\right)+1<\operatorname{dim}(X)$ e $G$ age livremente fora de $Y=\bigcup_{i=1}^{n} Y_{i}$.

Sejam A uma $G$-subvariedade fechada e não vazia de $X$ e contornável em $X, f: X \rightarrow$ $X$ uma G-aplicação tal que $A \subset F i x(f), f$ não possui pontos fixos em $Y-A$ e $f$ possui uma quantidade finita de pontos fixos em $X-(A \cup Y)$.

Sejam $x_{0}$ e $x_{1}$ dois pontos fixos de $f G$-Nielsen equivalentes e em órbitas diferentes tais que $x_{0} \in X-(A \cup Y)$ e $x_{1} \in X-(A \cup Y)$ ou $x_{1} \in \partial(A)$, onde $\partial(A)$ é o bordo de $A$ em $X$ e $q: I \rightarrow X$ um caminho de $q(0)=x_{0}$ até $q(1)=x_{1}$ tal que $f \circ q$ é homotópico a $q$ relativo aos extremos. Então $f$ é $G$-homotópica relativo a $(A \cup Y)$ a uma aplicação $h: X \rightarrow X$ tal que Fix $(h)=$ Fix $(f)-G\left\{x_{0}\right\}$.

Prova do Procedimento 2.9: Como $A$ é uma $G$-subvariedade fechada e não vazia de $X$ temos que $A$ é um $G$-subconjunto de $X$, localmente contrátil, fechado e não vazio. Assim as hipóteses do Procedimento 2.8 estão satisfeitas. 


\subsection{Realização de um Conjunto como Conjunto de Pon- tos Fixos de uma dada $G$-Aplicação}

Generalizamos nessa seção o Lema 3.1 de [9] e alguns resultados subsequentes. Lembramos que quando $G$ é um grupo de Lie compacto e $X$ uma $G$-variedade suave e compacta temos que $I s o(X)$ é finito. Daqui até o fim dessa seção estamos admitindo que $I s o(X)$ está munido de uma ordem admissível $\left\{\left(K_{i}\right)\right\}_{i=1}^{n}$, o que gera a filtração $X_{1} \subset \cdots \subset X_{n}=X$ de $G$-subespaços de $X$. O primeiro resultado que buscamos é o seguinte teorema:

Teorema 2.10 Sejam $G$ um grupo de Lie compacto, $X$ uma $G$-variedade suave e compacta, A um $G$-subconjunto de $X$ localmente contrátil, fechado e não vazio e $f: X \rightarrow X$ uma $G$-aplicação tais que para $W K$ finito temos que $\operatorname{dim}\left(X^{K}\right) \geq 3, \operatorname{dim}\left(X^{K}\right)-\operatorname{dim}\left(X^{K}\right.$ $\left.X_{K}\right) \geq 2$ e $A^{K}$ é contornável em $X^{K}$, para todo $(K) \in I$ so $(X)$.

Então $f$ é G-homotópica a uma $h: X \rightarrow X$ tal que Fix $(h)=A$ se, e somente se, as seguintes condições estiverem satisfeitas:

(C1) existe uma G-aplicação $\bar{H}:(X \times\{0\}) \cup(A \times I) \rightarrow X$ tal que $\bar{H}(\bullet, 0)=f$ e $\bar{H}(\bullet, 1)=i: A \hookrightarrow X($ inclusão $) ;$

(C2) sempre que $W K$ for finito, toda $W K$-classe de pontos fixos essencial $F$ de $f^{K}$ está conectada a $A^{K}$ por um caminho $p: I \rightarrow X^{K}\left(p(0) \in F\right.$ e $\left.p(1) \in A^{K}\right)$ tal que $\{p(t)\}$ é homotópica à $\left\{f^{K} \circ p\right\} *\left\{\bar{H}^{K}(p(1), t)\right\}$ com pontos extremos fixados.

Dividimos sua prova em casos particulares, os quais provamos separadamente, visando uma melhor análise das construções envolvidas. Observamos que se $A$ for uma $G$-subvariedade de $X$ então $A$ também é um $G$-subconjunto de $X$ localmente contrátil.

Lema 2.11 Sejam $G$ um grupo finito, $X$ uma $G$-variedade suave, compacta e $\operatorname{dim}(X)>$ 2, $\left\{Y_{i}\right\}_{i=1}^{n}$ uma coleção finita de G-subvariedades fechadas e não vazias de $X$ tal que $\operatorname{dim}\left(Y_{i}\right)+1<\operatorname{dim}(X)$ e $G$ age livremente fora de $Y=\bigcup_{i=1}^{n} Y_{i}$.

Sejam $A$ um $G$-subconjunto de $X$ localmente contrátil, fechado e não vazio tal que $A$ é contornável em $X$ e $f: X \rightarrow X$ uma $G$-aplicação tal que $\left.f\right|_{A}=i: A \hookrightarrow X$ (inclusão) e Fix $\left(\left.f\right|_{Y}\right)=A \cap Y$.

Suponhamos que toda $G$-classe de pontos fixos essencial $F$ de $f$ está conectada a $A$ por um caminho $p: I \rightarrow X, p(0) \in F$ e $p(1) \in A$, tal que $\{p(t)\}$ é homotópica à $\{f \circ p(t)\}$ com pontos extremos fixados. Então f é equivariantemente homotópica (relativo a $A \cup Y$ ) a uma G-aplicação $h: X \rightarrow X$ tal que Fix $(h)=A$.

Prova do Lema 2.11: Pela Proposição 2.3 existe uma $G$-triangulação de $X$ na qual $Y$ é um $G$-subpoliedro. Pela Proposição 2.4 podemos tomar essa triangularização de modo que $S t(A)$ seja contornável em $X$, e pela Proposição 2.2 existe $V$ uma vizinhança fechada e invariante de $S t(A) \cup Y$ em $X$ que se retrai equivariantemente em $S t(A) \cup Y$.

Segue do Lema 3.1 de [18] que existe uma $G$-homotopia $H_{1}: X \times I \rightarrow X$ relativa a $(S t(A) \cup Y)$, entre $f$ e uma aplicação $h_{1}=H_{1}(\bullet, 1): X \rightarrow X$ tal que: 
1. $A \subset F i x\left(h_{1}\right)$;

2. $(U-A) \cap F i x\left(h_{1}\right)=\emptyset$ para alguma uma vizinhança $G$-invariante $U$ de $(S t(A) \cup Y)$ em $X$;

3. $h_{1}$ possui uma quantidade finita de pontos fixos em $X-(A \cup Y)$ todos contidos no interior de simplexos maximais da triangularização de $X$.

Com o Procedimento 2.8 colapsamos todas as $G$-órbitas de pontos fixos de $h_{1}$ que são $G$-Nielsen equivalentes e que se encontram em $X-(S t(A) \cup Y)$, também colapsamos todas as $G$-órbitas de pontos fixos de $h_{1}$ que se encontram em $X-(S t(A) \cup Y)$ e que são $G$-Nielsen equivalentes a alguma $G$-órbita de pontos fixos que se encontra em $\partial(A)$ na $G$-órbita de pontos fixos de $\partial(A)$.

Assim, $h_{1}$ é $G$-homotópica a uma aplicação $h_{2}$ tal que dada uma classe de pontos fixos $F$ de $h_{2}$ podemos dizer que:

1. se $F \cap A \neq \emptyset$ então $F \subset A$;

2. se $F \cap A=\emptyset$ então $F=G\{x\} \operatorname{com} x \in X-(A \cup Y)$.

Todo ponto fixo de $h_{2}$ que está em $X-(S t(A) \cup Y)$ é isolado, assim usamos o Teorema VIII.B.4 (pg 123) de [1] para remover todas as $G$-órbitas de pontos fixos $F=G\{x\}$ de índice 0 de $h_{2}$ que não interceptam $A$. Cada vez que usamos o lema devemos estender a homotopia por equivariância da mesma forma que é feito na página 42.

Dessa forma, obtemos uma $G$-homotopia $H: X \times I \rightarrow X$ entre $h_{2}$ e uma $G$-aplicação $h=H(\bullet, 1)$ tal que $F i x(h)=A$. De fato, suponhamos que exista um ponto $x \in X$ fixado por $h$ tal que $G\{x\} \subset F i x(h)-A$, então $G\{x\}$ é uma $G$-classe essencial de pontos fixos de $h$ (isso se deve a forma como construímos $h$ ). Logo, $G\{x\}$ está $H$-relacionada com alguma $G$-classe de pontos fixos essencial $F$ de $f$.

Por hipótese existe um caminho $p: I \rightarrow X$ com $p(0) \in F, p(1) \in A$ e tal que $\{p(t)\}$ é homotópica à $\{f \circ p(t)\}$, o que significa que $F$ está $H$-relacionada com alguma $G$-classe de pontos fixos em $A$. Consequentemente, $p(1)=g x$, para algum $g \in G$, daí, $G\{x\} \subset A$, mas $G\{x\} \nsubseteq A$, o que é um absurdo. Portanto, Fix $(h)=A$.

O resultado anterior, por si só, pode ser aplicado em uma grande quantidade de problemas de realização de pontos fixos. Uma consequência dele é o seguinte corolário:

Corolário 2.12 Sejam $G$ um grupo finito, $X$ uma G-variedade suave, compacta tal que $\operatorname{dim}(X)>2,\left\{Y_{i}\right\}_{i=1}^{n}$ uma coleção finita de $G$-subvariedades fechadas e não vazias de $X$ tal que $\operatorname{dim}\left(Y_{i}\right)+1<\operatorname{dim}(X)$ e $G$ age livremente fora de $Y=\bigcup_{i=1}^{n} Y_{i}$.

Sejam $A$ uma $G$-subvariedade fechada e não vazia de $X$ tal que A é contornável em $X$ e $f: X \rightarrow X$ uma G-aplicação tal que $\left.f\right|_{A}=i: A \hookrightarrow X$ (inclusão) e Fix $\left(\left.f\right|_{Y}\right)=A \cap Y$.

Suponhamos que toda $G$-classe de pontos fixos essencial $F$ de $f$ está conectada a $A$ por um caminho $p: I \rightarrow X, p(0) \in F$ e $p(1) \in A$, tal que $\{p(t)\}$ é homotópica à $\{f \circ p(t)\}$ 
com pontos extremos fixados. Então $f$ é equivariantemente homotópica relativo a $A \cup Y$ a uma $G$-aplicação $h: X \rightarrow X$ tal que Fix $(h)=A$.

Prova do Corolário 2.12: Como $A$ é uma $G$-subvariedade fechada e não vazia de $X$ temos que $A$ é um $G$-subconjunto de $X$, localmente contrátil, fechado e não vazio. Assim as hipóteses do Procedimento 2.8 estão satisfeitas.

Lema 2.13 Com as hipóteses do teorema 2.10. Suponhamos que:

1. $W K_{i}$ é finito;

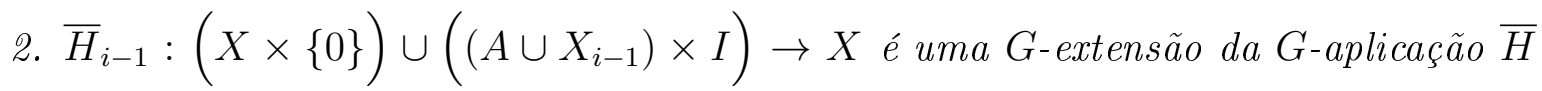
dada em C1 tal que Fix $\left(h_{i-1}\right)=A$, onde $h_{i-1}=\bar{H}_{i-1}(\bullet, 1): A \cup X_{i-1} \rightarrow X$.

Então existe uma $G$-extensão $\bar{H}_{i}:(X \times\{0\}) \cup\left(\left(A \cup X_{i}\right) \times I\right) \rightarrow X$ de $\bar{H}_{i-1}$ tal que Fix $\left(h_{i}\right)=A$, onde $h_{i}=\bar{H}_{i}(\bullet, 1): A \cup X_{i} \rightarrow X$.

Prova do Lema 2.13: $\left(X_{i},\left(A_{i} \cup X_{i-1}\right)\right)$ é um $G$-par metrizável e $X_{i}$ é um $G$-ANR, assim, a Proposição 9.3 de [22] garante que existe extensão:

$$
{ }_{1} \bar{H}_{i}:(X \times\{0\}) \cup\left(\left(A \cup X_{i}\right) \times I\right) \rightarrow X
$$

para a $G$-aplicação $\bar{H}_{i-1}$, pois $\left(X_{i}, A_{i} \cup X_{i-1}\right)$ é um $G$-par metrizável $\left(A_{i}=X_{i} \cap A\right)$ e $X_{i}$ é um $G$-ANR.

Daí, encontramos uma $G$-aplicação ${ }_{1} h_{i}={ }_{1} \bar{H}_{i}(\bullet, 1): A \cup X_{i} \rightarrow A \cup X_{i}$ tal que $A \subset$ Fix $\left({ }_{1} h_{i}\right)$. Para obter mais informações sobre os pontos fixos dessa aplicação em $X_{i}-\left(A \cup X_{i-1}\right)$ usaremos o Lema 2.11 dessa seção e o Lema 2.1 de [12].

Como foi visto anteriormente, $W K_{i}$ age livremente em $X_{i}^{K_{i}}-X_{i-1}$ e a aplicação ${ }_{1} h_{i}^{K_{i}}: X_{i}^{K_{i}} \rightarrow X_{i}^{K_{i}}$ é uma $W K_{i}$-aplicação. Pela Proposição 2.4 existe uma triangulação $W K_{i}$-equivariante de $X^{K_{i}}$ na qual $S t\left(A_{i}^{K_{i}}\right)$ seja contornável em $X_{i}^{K_{i}}$. Pela Proposição 2.2 existe $V$ uma vizinhança fechada e invariante de $S t\left(A_{i}\right) \cup X_{i-1}$ em $X_{i}$ que se retrai equivariantemente em $X_{i-1} \cup S t\left(A_{i}\right)$.

Portanto, as hipóteses do Lema 2.11 estão satisfeitas e podemos aplicá-lo relativo a $W K_{i}$-triangularização de $X_{i}^{K_{i}}$. Assim, obtemos uma $W K_{i}$-homotopia $H:\left(A \cup X_{i}\right)^{K_{i}} \times I \rightarrow$ $X^{K_{i}}$ entre ${ }_{1} h_{i}$ e a $W K_{i}$-aplicação $h=H(\bullet, 1)$ tal que $F i x(h)=A^{K_{i}}$.

Observamos que $\left(X_{i}, X_{i-1} \cup A_{i}\right)$ é um $G$-par tal que $X_{i}-\left(X_{i-1} \cup A_{i}\right)$ possui o mesmo tipo de órbita $\frac{G}{K_{i}}, V$ é uma $G$-vizinhança fechada de $X_{i-1} \cup A_{i}$ em $X_{i}$ e $\left.{ }_{1} h_{i}\right|_{X_{i}}: X_{i} \rightarrow A \cup X_{i}$ uma $G$-aplicação.

Dessa forma, com o Lema 2.1 de [12] estendemos essa $W K_{i}$-homotopia para uma $G$ aplicação $\bar{H}_{i}:(X \times\{0\}) \cup\left(\left(A \cup X_{i}\right) \times I\right) \rightarrow X$, relativo a $V$, e como $h$ não possui pontos fixos em $X_{i}-\left(X_{i-1} \cup A_{i}\right)$ temos que $F i x\left(h_{i}\right)=A$, onde $h_{i}=\bar{H}_{i}(\bullet, 1): A \cup X_{i} \rightarrow X$. 
Agora vamos estender a homotopia dada em $C 1$ do Teorema 2.10 com $W K$ um grupo de Lie compacto de dimensão positiva.

Lema 2.14 Com as hipóteses do teorema 2.10. Suponhamos que:

1. $W K_{i}$ é um grupo de Lie compacto de dimensão positiva;

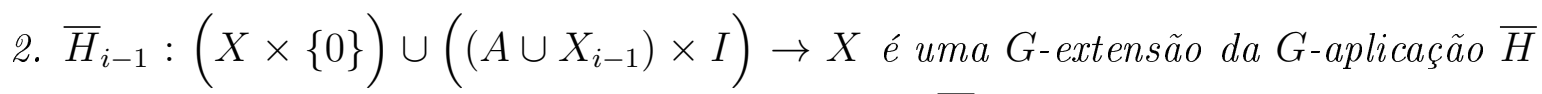
dada em C1 tal que Fix $\left(h_{i-1}\right)=A$, onde $h_{i-1}=\bar{H}_{i-1}(\bullet, 1): A \cup X_{i-1} \rightarrow X$.

Então existe uma $G$-extensão $\bar{H}_{i}:(X \times\{0\}) \cup\left(\left(A \cup X_{i}\right) \times I\right) \rightarrow X$ de $\bar{H}_{i-1}$ tal que Fix $\left(h_{i}\right)=A$, onde $h_{i}=\bar{H}_{i}(\bullet, 1): A \cup X_{i} \rightarrow X$.

Prova do Lema 2.14: $\left(X_{i},\left(A_{i} \cup X_{i-1}\right)\right)$ é um $G$-par metrizável e $X_{i}$ é um $G$-ANR, assim, usamos a Proposição 9.3 de [22] para estender $\bar{H}_{i-1}$ para uma $G$-aplicação

$$
{ }_{1} \bar{H}_{i}:(X \times\{0\}) \cup\left(\left(A \cup X_{i}\right) \times I\right) \rightarrow X .
$$

Daí, ${ }_{1} h_{i}={ }_{1} \bar{H}_{i}(\bullet, 1): A \cup X_{i} \rightarrow A \cup X_{i}$ é uma $G$-aplicação tal que $A \subset F i x\left({ }_{1} h_{i}\right)$. Pela Proposição 2.2, existe $V$ uma vizinhança fechada e invariante de $A_{i} \cup X_{i-1}$ em $X_{i}$ que se retrai equivariantemente em $X_{i-1} \cup A_{i}$.

Logo, $W K_{i}$ é um grupo de Lie compacto, cada componente conexa de $X_{i}^{K_{i}}$ é um $W K_{i^{-}}$ espaço compacto, $W K_{i}$ age livremente em $X_{i}^{K_{i}}-X_{i-1}$ e a aplicação ${ }_{1} h_{i}^{K_{i}}: X_{i}^{K_{i}} \rightarrow X_{i}^{K_{i}}$ é uma $W K_{i}$-aplicação.

Dessa forma, segue do Lema 3.3 de [18] que existe uma $W K_{i}$-homotopia $H: X_{i}^{K_{i}} \times I \rightarrow$ $X^{K_{i}}$ relativa a $\left(A_{i} \cup X_{i-1}\right)^{K_{i}}$, entre ${ }_{1} h_{i}^{K_{i}}$ e a aplicação $h=H(\bullet, 1)$ que não possui pontos fixos em $X_{i}^{K_{i}}-\left(A_{i} \cup X_{i-1}\right)^{K_{i}}$.

Segue que $\left(X_{i}, X_{i-1} \cup A_{i}\right)$ é um $G$-par tal que $X_{i}-\left(X_{i-1} \cup A_{i}\right)$ possui o mesmo tipo de órbita $\frac{G}{K_{i}}, V$ é uma $G$-vizinhança fechada de $X_{i-1} \cup A_{i}$ em $X_{i}$ e $\left.{ }_{1} h_{i}\right|_{X_{i}}: X_{i} \rightarrow A \cup X_{i}$ é uma $G$-aplicação. Com o lema 2.1 de [12] estendemos a $W K_{i}$-homotopia $H:\left(A \cup X_{i}\right)^{K_{i}} \times I \rightarrow$ $X^{K_{i}}$ para uma $G$-aplicação $\bar{H}_{i}:(X \times\{0\}) \cup\left(\left(A \cup X_{i}\right) \times I\right) \rightarrow X$, relativo a $V$. Além disso, $h$ não possui pontos fixos em $X_{i}-\left(X_{i-1} \cup A_{i}\right)$, consequentemente, Fix $\left(h_{i}\right)=A$, onde $h_{i}=\bar{H}_{i}(\bullet, 1): A \cup X_{i} \rightarrow X$.

Prova do Teorema 2.10: Se $f$ é G-homotópica a uma $h: X \rightarrow X$ tal que $F i x(h)=$ $A$, então existe uma $G$-homotopia $H: X \times I \rightarrow X$ entre $f$ e $h$ tal que sua restrição $\bar{H}=\left.H\right|_{(X \times\{0\}) \cup(A \times I)}$ satisfaz $(C 1)$.

Se $F$ for uma $W K$-classe de pontos fixos essencial de $f^{K}$, para $W K$ finito, então existe um caminho $p: I \rightarrow X^{K}$ tal que $p(0) \in F$ e $p(1) \in J$, onde $J \subset A^{K}$ é a $W K$-classe de 
pontos fixos essencial de $h^{K}$ que está $H^{K}$-relacionada com $F$ e $\{p(t)\}$ é homotópica à $\left\{\bar{H}^{K}(p(t), t)\right\}$ (com pontos extremos fixados). Como $\left\{\bar{H}^{K}(p(t), t)\right\}$ é homotópica (com pontos extremos fixados) à:

$$
\underbrace{\left\{\bar{H}^{K}(p(t), 0)\right\}}_{=\{f \circ p(t)\}} *\left\{\bar{H}^{K}(p(1), t)\right\} .
$$

Dessa forma, temos que $(C 2)$ está satisfeito.

Suponhamos que $(C 1)$ e $(C 2)$ são válidos e consideremos a $G$-aplicação $\bar{H}:(X \times$ $\{0\}) \cup(A \times I) \rightarrow X$ tal que $\bar{H}(\bullet, 0)=f$ e $\bar{H}(\bullet, 1)=i: A \hookrightarrow X$ (inclusão) dada em $(C 1)$. Usamos a Proposição 9.3 de [22] e estendemos $\bar{H}$ para uma $G$-aplicação:

$$
{ }_{1} \bar{H}_{1}:(X \times\{0\}) \cup\left(\left(A \cup X_{1}\right) \times I\right) \rightarrow X,
$$

pois $\left(X_{1}, A_{1}\right)$ é um $G$-par metrizável $\left(A_{1}=X_{1} \cap A\right)$ e $X_{1}$ é um $G$-ANR.

Pela Proposição 2.2 existe $V$ uma vizinhança fechada e invariante de $A_{1}$ em $X_{1}$ que se retrai equivariantemente em $A_{1}$. Temos que $W K_{1}$ age livremente em $X_{1}^{K_{1}}=X_{K_{1}}$ e a aplicação ${ }_{1} h_{1}^{K_{1}}$ é uma $W K_{1}$-aplicação. Pela Proposição 2.3 existe uma triangulação $W K_{1}$-equivariante de $X^{K_{1}}$ na qual $A_{1}^{K_{1}}$ é um $W K_{1}$-subpoliedro.

Portanto, se $W K_{1}$ for finito as hipóteses do Lema 2.11 estão satisfeitas e podemos aplicá-lo em $X_{1}^{K_{1}}$ relativo a $W K_{1}$-triangularização de $X_{1}^{K_{1}}$ e a vizinhança $V^{K_{1}} W K_{1}$ invariante de $A_{1}^{K_{1}}$, pois $W K_{1}$ é um grupo finito, $X_{1}^{K_{1}}$ é um $W K_{1}$-espaço compacto e ${ }_{1} h_{1}^{K_{1}}$ é uma $W K_{i}$-aplicação.

Assim, obtemos uma $W K_{1}$-homotopia $H:\left(A_{1} \cup X_{1}\right)^{K_{1}} \times I \rightarrow X^{K_{1}}$ entre ${ }_{1} h_{1}$ e a $W K_{1}$-aplicação $k=H(\bullet, 1)$ tal que $F i x(k)=A_{1}^{K_{1}}$ e estendemos a $W K_{1}$-homotopia $H:\left(A \cup X_{1}\right)^{K_{1}} \times I \rightarrow X^{K_{1}}$ para uma $G$-aplicação:

$$
\bar{H}_{1}:(X \times\{0\}) \cup\left(\left(A \cup X_{1}\right) \times I\right) \rightarrow X,
$$

relativo a $V$, usando o Lema 2.1 de [12]. Isso pode ser feito porque $\left(X_{1}, A_{1}\right)$ é um $G$-par tal que $X_{1}-A_{1}$ possui o mesmo tipo de órbita $\frac{G}{K_{1}}, V$ é uma $G$-vizinhança fechada de $A_{1}$ em $X_{1}$ e $\left.{ }_{1} h_{1}\right|_{X_{1}}: X_{1} \rightarrow A \cup X_{1}$ uma $G$-aplicação.

Como $k$ não possui pontos fixos em $X_{1}-A_{1}$ temos que Fix $\left(h_{1}\right)=A$, onde $h_{1}=$ $\bar{H}_{1}(\bullet, 1): A \cup X_{1} \rightarrow X$.

Se $W K_{1}$ for um grupo de Lie compacto de dimensão positiva, aplicamos o Lema 3.3 de [18] em $X_{1}^{K_{1}}$ relativamente a $W K_{1}$-vizinhança $V^{K_{1}}$ de $A_{1}^{K_{1}}$ que se retrai $W K_{1}$ equivariantemente em $A_{1}^{K_{1}}$.

Assim, encontramos uma $W K_{1}$-homotopia $H: X_{1}^{K_{1}} \times I \rightarrow X^{K_{1}}$ relativa a $A_{1}^{K_{1}}$, entre ${ }_{1} h_{1}^{K_{1}}$ e a aplicação $h=H(\bullet, 1)$ que não possui pontos fixos em $X_{1}^{K_{1}}-A_{1}^{K_{1}}$. Daí, usamos o lema 2.1 de [12] para estendemos a $W K_{1}$-homotopia $H:\left(A \cup X_{1}\right)^{K_{1}} \times I \rightarrow X^{K_{1}}$ para uma $G$-aplicação $\bar{H}_{1}:(X \times\{0\}) \cup\left(\left(A \cup X_{1}\right) \times I\right) \rightarrow X$, relativo a $V$. Além 
disso, $h$ não possui pontos fixos em $X_{1}-A_{1}$, consequentemente, Fix $\left(h_{1}\right)=A$, onde $h_{1}=\bar{H}_{1}(\bullet, 1): A \cup X_{1} \rightarrow X$.

Por indução finita, suponhamos que estendemos a $G$-aplicação $\bar{H}$ dada em $C 1$ para uma $G$-aplicação $\bar{H}_{i-1}:(X \times\{0\}) \cup\left(\left(A \cup X_{i-1}\right) \times I\right) \rightarrow X$ tal que Fix $\left(h_{i-1}\right)=A$, onde $h_{i-1}=\bar{H}_{i-1}(\bullet, 1): A \cup X_{i-1} \rightarrow X$. O índice $i$ percorre o mesmo conjunto finito de índices de $I s o(X)$.

Quando $W K_{i}$ for finito podemos usar o Lema 2.13 para estender a $G$-aplicação $\bar{H}_{i-1}$ para uma $G$-aplicação $\bar{H}_{i}:(X \times\{0\}) \cup\left(\left(A \cup X_{i}\right) \times I\right) \rightarrow X$ com as propriedades de $F i x\left(h_{i}\right)=A$, onde $h_{i}=\bar{H}_{i}(\bullet, 1): A \cup X_{i} \rightarrow X$.

Quando $W K_{i}$ tiver dimensão positiva podemos usar o Lema 2.14 para estende a $G$ aplicação $\bar{H}_{i-1}$ para uma $G$-aplicação $\bar{H}_{i}:(X \times\{0\}) \cup\left(\left(A \cup X_{i}\right) \times I\right) \rightarrow X$ com as propriedades de $\operatorname{Fix}\left(h_{i}\right)=A$, onde $h_{i}=\bar{H}_{i}(\bullet, 1): A \cup X_{i} \rightarrow X$.

Observando que se $A$ for uma $G$-subvariedade fechada e não vazia de $X$ temos que $A$ é um $G$-subconjunto de $X$, localmente contrátil, fechado e não vazio, o que satisfaz as hipóteses do Teorema 2.10. Dessa forma, temos o seguinte corolário:

Corolário 2.15 Sejam $G$ um grupo de Lie compacto, $X$ uma $G$-variedade suave e compacta, $A$ uma $G$-subvariedade de $X$, fechada e não vazia e $f: X \rightarrow X$ uma $G$-aplicação tais que para $W K$ finito temos que $\operatorname{dim}\left(X^{K}\right) \geq 3, \operatorname{dim}\left(X^{K}\right)-\operatorname{dim}\left(X^{K}-X_{K}\right) \geq 2$ e $A^{K}$ é contornável em $X^{K}$, para todo $(K) \in I s o(X)$.

Então $f$ é G-homotópica a uma $h: X \rightarrow X$ tal que Fix $(h)=A$ se, e somente se, as seguintes condições estiverem satisfeitas:

(C1) existe uma $G$-aplicação $\bar{H}:(X \times\{0\}) \cup(A \times I) \rightarrow X$ tal que $\bar{H}(\bullet, 0)=f$ e $\bar{H}(\bullet, 1)=i: A \hookrightarrow X($ inclusão $) ;$

(C2) sempre que $W K$ for finito, toda $W K$-classe de pontos fixos essencial $F$ de $f^{K}$ está conectada a $A^{K}$ por um caminho $p: I \rightarrow X^{K}\left(p(0) \in F\right.$ e $\left.p(1) \in A^{K}\right)$ tal que $\{p(t)\}$ é homotópica à $\left\{f^{K} \circ p\right\} *\left\{\bar{H}^{K}(p(1), t)\right\}$ com pontos extremos fixados.

O Corolário 2.16 se refere ao teorema 2.10 para o caso particular $G$ grupo finito. A maior diferença entre o próximo resultado e os outros resultados dessa seção é que agora realizaremos, sobre certas hipóteses, qualquer $G$-subconjunto fechado que esteja dentro do $G$-subconjunto localmente contrátil.

Corolário 2.16 Sejam $G$ um grupo finito, $X$ uma $G$-variedade suave e compacta com $\operatorname{dim}\left(X^{K}\right) \geq 3$ e $\operatorname{dim}\left(X^{K}\right)-\operatorname{dim}\left(X^{K}-X_{K}\right) \geq 2$ e $\Phi$ um G-subconjunto fechado, não vazio e localmente contrátil de $X$ tais que $\Phi^{K}$ é contornável em $X^{K}$, onde $(K) \in I s o(X)$.

Sejam A um $G$-subconjunto fechado e não vazio de $\Phi$ que intersecta todas as componentes conexas de $\Phi$ e $f: X \rightarrow X$ uma G-aplicação. Então $f$ é G-homotópica a uma $h: X \rightarrow X$ tal que Fix $(h)=A$ se as seguintes condições estiverem satisfeitas: 
(C1) existe uma $G$-aplicação $\bar{H}:(X \times\{0\}) \cup(\Phi \times I) \rightarrow X$ tal que $\bar{H}(\bullet, 0)=f$ e $\bar{H}(\bullet, 1)=i: \Phi \hookrightarrow X($ inclusão).

(C2) toda WK-classe de pontos fixos essencial $F$ de $f^{K}$ está conectada a $\Phi^{K}$ por um caminho $p: I \rightarrow X^{K}\left(p(0) \in F\right.$ e p $\left.(1) \in \Phi^{K}\right)$ tal que $\{p(t)\}$ é homotópica à $\left\{f^{K} \circ p\right\} *\left\{\bar{H}^{K}(p(1), t)\right\}$ (com pontos extremos fixados).

Prova do Corolário 2.16: Segue do teorema 2.10 que existe uma $G$-aplicação $h_{1}$ : $X \rightarrow X$ equivariantemente homotópica a $f$ tal que $F i x\left(h_{1}\right)=\Phi$.

Primeiramente, queremos encontrar uma $G$-aplicação $h_{2}$ equivariantemente homotópica a $h_{1}$ tal que $h_{2}$ seja uma $G$-aplicação proximal em $S t(\Phi)$ e com uma quantidade finita de pontos fixos todos em $\Phi$ de modo que cada componente de $\Phi$ possua pelo menos um ponto fixo.

Com as Proposições 2.2 e 2.3 equipamos $X$ com uma $G$-triangularização tal que $\operatorname{St}(\Phi)$ seja um $G$-subpoliedro de $X$ e para garantir a existência de uma vizinhança invariante $V$ de $S t(\Phi)$ que se retrai equivariantemente em $S t(\Phi)$. Aplicando a Proposição 2.5 de [18] em $h_{1}$ com relação a $\Phi$, para um $\epsilon>0$ suficientemente pequeno, encontramos uma $G$-aplicação $h_{1}^{\prime}$ tal que $F i x\left(h_{1}^{\prime}\right)=\Phi$ e existe uma vizinhança $U$ de $S t(\Phi)$ tal que $\left.h_{1}^{\prime}\right|_{U}=\left.h_{1}^{\prime}\right|_{\Phi} \circ r$, onde $r: U \rightarrow \Phi$ é uma retração equivariante.

Podemos tomar um refinamento da triangularização de $X$ se necessário para garantir que $h_{1}^{\prime}$ é proximal em $S t(\Phi)$. Depois, aplicamos o Teorema 4.3 de [13], para um $\epsilon>0$ suficientemente pequeno, para encontrar uma $G \epsilon$-homotopia $H_{2}: X \times I \rightarrow X$ entre $h_{1}^{\prime}$ e uma $G$-aplicação $h_{2}$ tal que $\left.h_{2}\right|_{X_{K}}$ possui um número finito de pontos fixos todos contidos em simplexos maximais de $X^{K}$, para cada $(K) \in I s o(X)$, e $\epsilon$ pode ser tomado suficientemente pequeno para que $h_{2}$ seja uma $G$-aplicação proximal em $S t(\Phi)$.

Como $A$ intersecta todas as componentes de $\Phi$ podemos puxar todos os pontos fixos de $h_{1}$ para o bordo de $A$. Assim, $h_{2}$ é $G$-homotópica a uma aplicação $h_{3}: X \rightarrow X$ tal que $F i x\left(h_{3}\right) \subset A$ (cada componente de $A$ possui pelo menos um ponto fixo) e $h_{3}$ é proximal em $\operatorname{St}(\Phi)$.

O Lema 1.3 de [15] diz que a aplicação $\alpha$ dada no Lema VIII.C.1 da página 124 de [1] é equivariante. Dessa forma, tomemos a métrica equivariante $d: X \times X \rightarrow \mathbb{R}$ e definimos a aplicação:

$$
\begin{aligned}
\overline{h_{4}}: S t(\Phi) & \rightarrow S t(\Phi) \\
x & \mapsto \alpha\left(x, h_{3}(x), \bar{d}(x, A)\right),
\end{aligned}
$$

onde $\bar{d}$ é a métrica limitada relativa a $d$.

Consideremos a $G$-aplicação $\bar{H}_{4}:(X \times\{0\}) \cup(S t(\Phi) \times I) \rightarrow X$ dada por:

$$
(x, t) \mapsto \begin{cases}\alpha\left(x, h_{3}(x), 1-(1-\bar{d}(x, A)) t\right) & \text { se }(x, t) \in S t(\Phi) \times I \\ h_{3}(x) & \text { se } t=0\end{cases}
$$

Para terminar essa demonstração basta estender essa homotopia sem criar novos pontos fixos. Para isso, considere a seguinte $G$-aplicação: 


$$
\begin{aligned}
H_{4}^{\prime}: & ((X-\operatorname{Int}(S t(\Phi))) \times\{0\}) \cup(\partial(S t(\Phi)) \times I) \rightarrow X \\
& (x, t) \mapsto \begin{cases}\bar{H}_{4}(x, t) & \text { se } t \neq 0 \\
h_{3}(x) & \text { se } t=0\end{cases}
\end{aligned}
$$

Pela Proposição 9.3 de [22] estendemos $H_{4}^{\prime}$ para uma $G$-aplicação:

$$
\bar{H}_{4}^{\prime}:(X-\operatorname{Int}(\operatorname{St}(\Phi))) \times I \rightarrow X .
$$

Caso exista algum ponto fixo em $\bar{H}_{4}^{\prime}(X-S t(\Phi) \times\{1\})$ usamos o Lema 3.1 de [18] em $\bar{H}_{4}^{\prime}(\bullet, 1)$ relativamente a $\partial(S t(\Phi))$ para reduzi-los a uma quantidade finita. Daí, podemos eliminar esses pontos fixos, pois serão todos oriundos de classes não essenciais (aparecem durante a homotopia) uma vez que $\left.h_{3}\right|_{X-I n t(\Phi)}$ é livre de pontos fixos. Assim, $h_{3}$ é $G$ homotópica a uma aplicação $h_{4}$ tal que $F i x\left(h_{4}\right)=A$. A $G$-homotopia é dada por:

$$
\begin{aligned}
H_{4}: X \times I & \rightarrow X \\
(x, t) & \mapsto \begin{cases}\bar{H}_{4}(x, t) & \text { se } x \in S t(\Phi) \\
\bar{H}_{4}^{\prime}(x, t) & \text { se } x \notin S t(\Phi)\end{cases}
\end{aligned}
$$

O próximo corolário é a última consideração que faremos nessa seção a respeito das $G$-subvariedades.

Corolário 2.17 Sejam $G$ um grupo finito, $X$ uma $G$-variedade suave e compacta com $\operatorname{dim}\left(X^{K}\right) \geq 3$ e $\operatorname{dim}\left(X^{K}\right)-\operatorname{dim}\left(X^{K}-X_{K}\right) \geq 2$ e $\Phi$ uma $G$-subvariedade fechada e não vazia de $X$ tais que $\Phi^{K}$ é contornável em $X^{K}$, onde $(K) \in I s o(X)$.

Sejam A um $G$-subconjunto fechado e não vazio de $\Phi$ que intersecta todas as componentes conexas de $\Phi$ e $f: X \rightarrow X$ uma G-aplicação. Então $f$ é G-homotópica a uma $h: X \rightarrow X$ tal que Fix $(h)=A$ se as seguintes condiçôes estiverem satisfeitas:

(C1) existe uma $G$-aplicação $\bar{H}:(X \times\{0\}) \cup(\Phi \times I) \rightarrow X$ tal que $\bar{H}(\bullet, 0)=f e$ $\bar{H}(\bullet, 1)=i: \Phi \hookrightarrow X($ inclusão $)$.

(C2) toda WK-classe de pontos fixos essencial $F$ de $f^{K}$ está conectada a $\Phi^{K}$ por um caminho $p: I \rightarrow X^{K}\left(p(0) \in F\right.$ e $\left.p(1) \in \Phi^{K}\right)$ tal que $\{p(t)\}$ é homotópica à $\left\{f^{K} \circ p\right\} *\left\{\bar{H}^{K}(p(1), t)\right\}$ com pontos extremos fixados. 


\section{Capítulo 3}

\section{Pontos Fixos de Aplicações que Preservam Fibra}

O problema de realizar um conjunto como conjunto de pontos fixos de uma dada aplicação, inicialmente apresentado por H.Schirmer em [9], foi generalizado em [19] para o contexto dos fibrados com aplicações que preservam fibra.

O objetivo desse capítulo será generalizar o Teorema 4.1 de [19] para variedades equivariantes e conjuntos localmente contráteis.

Seguiremos nesse capítulo uma sequência parecida com a do capítulo anterior. Na primeira seção apresentaremos condições análogas as dadas por H. Schirmer em [9], provaremos sua necessidade e apresentaremos alguns exemplos que ilustram a diferença entre os elementos do capítulo anterior e desse. Na segunda seção faremos alguns lemas que usaremos na demonstração da generalização do Teorema 4.1 de [19] situada na terceira seção. $\mathrm{Na}$ terceira seção também faremos alguns corolários que trazem contextos particulares. 


\subsection{Exemplos Relacionados a Aplicações que Preser- vam Fibra}

No capítulo anterior encontramos condições para realizar um conjunto invariante como o conjunto de pontos fixos de uma aplicação equivariantemente homotópica a uma aplicação equivariante dada. Olhemos novamente para o exemplo 3 que demos na primeira seção do capítulo 2:

Tomamos $G=\mathbb{Z}_{2}, X=\mathbb{S}^{2} \times \mathbb{S}^{1}$, a ação é o conjugado na segunda coordenada:

$$
\xi((a, b, c), \cos (x)+i \operatorname{sen}(x)) \mapsto((a, b, c), \cos (x)-i \operatorname{sen}(x)) .
$$

Usamos a aplicação antípoda em $\mathbb{S}^{2}$ e a identidade em $\mathbb{S}^{1}$ :

$$
f((a, b, c), \cos (x)+i \operatorname{sen}(x))=((-a,-b,-c), \cos (x)+i \operatorname{sen}(x)),
$$

e $A_{1}=\left\{(a, b, 0) \in \mathbb{S}^{2}\right\} \times\{-i, i\}$. Daí, a homotopia que realiza $A_{1}$ como o conjunto de pontos fixos de uma aplicação equivariantemente homotópica a $f$ é:

$$
\begin{gathered}
H((\cos (\theta) \operatorname{sen}(\psi), \operatorname{sen}(\theta) \operatorname{sen}(\psi), \cos (\psi)), \cos (x)+i \operatorname{sen}(x), t)= \\
((-\cos (\theta+t|\operatorname{sen}(x)| \pi) \operatorname{sen}(\psi),-\operatorname{sen}(\theta+t|\operatorname{sen}(x)| \pi) \operatorname{sen}(\psi),-\cos (\psi)), \cos (x)+i \operatorname{sen}(x)) .
\end{gathered}
$$

Assim, a aplicação $h$ cujo conjunto de pontos fixos é $A_{1}$ é:

$$
\begin{gathered}
h((\cos (\theta) \operatorname{sen}(\psi), \operatorname{sen}(\theta) \operatorname{sen}(\psi), \cos (\psi)), \cos (x)+i \operatorname{sen}(x))= \\
((-\cos (\theta+|\operatorname{sen}(x)| \pi) \operatorname{sen}(\psi),-\operatorname{sen}(\theta+|\operatorname{sen}(x)| \pi) \operatorname{sen}(\psi),-\cos (\psi)), \cos (x)+i \operatorname{sen}(x)) .
\end{gathered}
$$

Agora, vamos agregar a essa estrutura a projeção na primeira coordenada $p: \mathbb{S}^{2} \times \mathbb{S}^{1} \rightarrow$ $\mathbb{S}^{2}$. Usando a ação trivial em $\mathbb{S}^{2}$ temos que $p$ é uma aplicação equivariante, $f$ é uma aplicação que preserva fibra e que $\bar{f}$ é a aplicação antípoda $a$. Dessa forma, temos o seguinte diagrama comutativo:

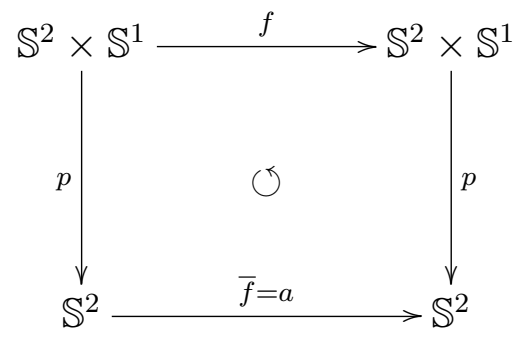

Observamos que:

$$
\begin{aligned}
p((x, y, z), \underbrace{\cos (0)+i \operatorname{sen}(0)}_{=1}) & =(x, y, z) \\
& =p((x, y, z), \underbrace{\cos \left(\frac{\pi}{2}\right)+i \operatorname{sen}\left(\frac{\pi}{2}\right)}_{=i}), \\
p \circ h((x, y, z), 1) & =(-x,-y,-z) .
\end{aligned}
$$


Contudo,

$$
\begin{gathered}
p \circ h((\cos (\theta) \operatorname{sen}(\psi), \operatorname{sen}(\theta) \operatorname{sen}(\psi), \cos (\psi)), i)= \\
(-\cos (\theta+\pi) \operatorname{sen}(\psi),-\operatorname{sen}(\theta+\pi) \operatorname{sen}(\psi),-\cos (\psi))= \\
(\cos (\theta) \operatorname{sen}(\psi), \operatorname{sen}(\theta) \operatorname{sen}(\psi),-\cos (\psi)),
\end{gathered}
$$

ou seja,

$$
p \circ h((x, y, z), i)=(x, y,-z) .
$$

Logo, $p((x, y, z), 1)=p((x, y, z), i)$ mas $p \circ h((x, y, z), 1)=(-x,-y,-z)$ que é quase sempre diferente de $p \circ h((x, y, z), i)=(x, y,-z)$, o que prova que $h$ não é uma aplicação que preserva fibra.

Nesse capítulo queremos encontrar um resultado que dê condições para realizar um conjunto invariante como o conjunto de pontos fixos de uma aplicação que preserva fibra equivariantemente homotópica por uma homotopia que preserva fibra a uma aplicação equivariante que preserva fibra dada.

Notemos que se $G$ for um grupo de Lie compacto, $\mathfrak{F}=(X, p, B, Y)$ um $G$-fibrado onde $X, B$ e $Y$ são $G$-espaços, $f: X \rightarrow X$ uma $G$-aplicação que preserva fibra e $A$ um subespaço fechado e $G$-invariante de $X$ então as condições $(C 1)$ e $(C 2)$ podem ser generalizadas para o contexto de aplicações que preservam fibras da seguinte forma:

$(C 1)_{\mathfrak{F}}$ existe uma $G$-homotopia parcial que preserva fibra $H_{A}:(X \times\{0\}) \cup(A \times I) \rightarrow X$ tal que $H_{A}(\bullet, 0)=f$ e $H_{A}(\bullet, 1)=i: A \hookrightarrow X$ (inclusão);

$(C 2)_{\mathfrak{F}}$ toda $W K$-classe de pontos fixos essencial $F$ de $f^{K}$ está conectada a $A^{K}$ por um caminho $\gamma: I \rightarrow X^{K}$ tal que $\gamma(0) \in F, \gamma(1) \in A^{K}$ e $\{\gamma(t)\}$ é homotópica à $\left\{f^{K} \circ \gamma\right\} *\left\{H_{A}^{K}(\gamma(1), t)\right\}$ com pontos extremos fixados.

Nesse sentido temos o seguinte lema:

Lema 3.1 Sejam $G$ um grupo de Lie compacto, $\mathfrak{F}=(X, p, B, Y)$ um $G$-fibrado onde $X$, $B$ e $Y$ são $G$-espaços $A N R, f: X \rightarrow X$ uma $G$-aplicação que preserva fibra e $A$ um subespaço fechado e $G$-invariante de $X$. Suponhamos que exista uma $G$-aplicação que preserva fibra $h: X \rightarrow X$ G-homotópica a $f$, por uma homotopia que preserva fibra, tal que Fix $(h)=A$ então $C 1_{\mathfrak{F}}$ e $C 2_{\mathfrak{F}}$ são válidas.

Prova do Lema 3.1: Existe uma G-homotopia que preserva fibras $H: X \times I \rightarrow X$ entre $f$ e $h$ tal que sua restrição $\bar{H}=\left.H\right|_{(X \times\{0\}) \cup(A \times I)}$ satisfaz $(C 1)_{\mathfrak{F}}$.

Se $F$ for uma $W K$-classe de pontos fixos essencial de $f^{K}$, para $W K$ finito, então existe um caminho $p: I \rightarrow X^{K}$ tal que $p(0) \in F$ e $p(1) \in J$, onde $J \subset A^{K}$ é a $W K$-classe de pontos fixos essencial de $h^{K}$ que está $H^{K}$-relacionada com $F$ e $\{p(t)\}$ é homotópica à $\left\{\bar{H}^{K}(p(t), t)\right\}$ (com pontos extremos fixados). Como $\left\{\bar{H}^{K}(p(t), t)\right\}$ é homotópica (com pontos extremos fixados) à:

$$
\underbrace{\left\{\bar{H}^{K}(p(t), 0)\right\}}_{=\left\{f^{K} \circ p(t)\right\}} *\left\{\bar{H}^{K}(p(1), t)\right\},
$$


temos que $(C 2)_{\mathfrak{F}}$ está satisfeita.

Voltemos para o exemplo 3 , o segundo conjunto que realizamos foi $A_{2}=\mathbb{S}^{1} \times \mathbb{S}^{1}$ com a homotopia dada por:

$$
\begin{gathered}
H_{2}((\cos (\theta) \operatorname{sen}(\psi), \operatorname{sen}(\theta) \operatorname{sen}(\psi), \cos (\psi)), \cos (x)+i \operatorname{sen}(x), t)= \\
((-\cos (\theta+t(1-|\cos (\psi)|) \pi) \operatorname{sen}(\psi),-\operatorname{sen}(\theta+t(1-|\cos (\psi)|) \pi) \operatorname{sen}(\psi),-\cos (\psi)), \\
\cos ((1-t) x+t x(1-|\cos (\psi)|))+i \operatorname{sen}((1-t) x+t x(1-|\cos (\psi)|))) .
\end{gathered}
$$

Nesse caso, temos que:

$$
\begin{gathered}
p \circ H_{2}((\cos (\theta) \operatorname{sen}(\psi), \operatorname{sen}(\theta) \operatorname{sen}(\psi), \cos (\psi)), \cos (x)+i \operatorname{sen}(x), t)= \\
p((-\cos (\theta+t(1-|\cos (\psi)|) \pi) \operatorname{sen}(\psi),-\operatorname{sen}(\theta+t(1-|\cos (\psi)|) \pi) \operatorname{sen}(\psi),-\cos (\psi)), \\
\cos ((1-t) x+t x(1-|\cos (\psi)|))+i \operatorname{sen}((1-t) x+t x(1-|\cos (\psi)|)))= \\
(-\cos (\theta+t(1-|\cos (\psi)|) \pi) \operatorname{sen}(\psi),-\operatorname{sen}(\theta+t(1-|\cos (\psi)|) \pi) \operatorname{sen}(\psi),-\cos (\psi)) .
\end{gathered}
$$

não depende de $x$. Assim, se

$$
p((\cos (\theta) \operatorname{sen}(\psi), \operatorname{sen}(\theta) \operatorname{sen}(\psi), \cos (\psi)), \cos (x)+i \operatorname{sen}(x))
$$

for igual a

$$
p((\cos (\phi) \operatorname{sen}(\rho), \operatorname{sen}(\phi) \operatorname{sen}(\rho), \cos (\rho)), \cos (y)+i \operatorname{sen}(y))
$$

então $\cos (\psi)=\cos (\rho), \operatorname{sen}(\psi)=\operatorname{sen}(\rho), \cos (\theta)=\cos (\phi)$ e $\operatorname{sen}(\theta)=\operatorname{sen}(\phi)$. Consequentemente,

$$
(-\cos (\theta+t(1-|\cos (\psi)|) \pi) \operatorname{sen}(\psi),-\operatorname{sen}(\theta+t(1-|\cos (\psi)|) \pi) \operatorname{sen}(\psi),-\cos (\psi))
$$

é igual a

$$
(-\cos (\phi+t(1-|\cos (\rho)|) \pi) \operatorname{sen}(\rho),-\operatorname{sen}(\phi+t(1-|\cos (\rho)|) \pi) \operatorname{sen}(\rho),-\cos (\rho)) .
$$

Portanto, $H_{2}$ é uma homotopia que preserva fibra e realizamos $A_{2}$ como o conjunto de pontos fixos de $h_{2}=H_{2}(\bullet, 1)$, uma aplicação que preserva fibra, que é equivariantemente homotópica por $H_{2}$, uma homotopia que preserva fibra, a $f$. 


\subsection{Resultados Preliminares para Aplicações que Pre- servam Fibra}

Nessa seção faremos observações e demonstraremos alguns resultados que usaremos nesse capítulo. Também criaremos alguns exemplos para elucidar algumas propriedades importantes.

O próximo resultado é uma generalização do Lema 2.1 de [12] oriunda de uma observação feita em sua demonstração.

Proposição 3.2 Sejam $G$ um grupo de Lie compacto, $(X, A)$ um $G$-par de espaços tal que todas as órbitas em $X-A$ possuem o mesmo tipo de órbita $(H)$. Sejam $f:(X, A) \rightarrow$ $(X, A)$ uma $G$-aplicação, $V$ uma $G$-vizinhança fechada de $A$ e $f^{H}: X^{H} \rightarrow X^{H}$ a restrição de $f$ a $X^{H}$.

Então qualquer $W H$-homotopia $f_{t}^{H}$, relativa a $V^{H}$, com $f_{0}^{H}=f^{H}$ se estende para uma $G$-homotopia $f_{t}$, relativa a $V$, com $f_{0}=f$. Além disso, se $f_{1}^{H}$ é livre de pontos fixos em $(X-A)^{H}$, então $f_{1}$ é livre de pontos fixos em $X-A$, e se Fix $\left(\left.f_{1}^{H}\right|_{(X-A)^{H}}\right)=$ $W H\left\{b_{1}\right\} \cup \cdots \cup W H\left\{b_{n}\right\}$, então Fix $\left(\left.f_{1}\right|_{X-A}\right)=G\left\{b_{1}\right\} \cup \cdots \cup G\left\{b_{n}\right\}$.

Prova da Proposição 3.2: Definimos a homotopia por:

$$
f_{t}(x)= \begin{cases}g f_{t}^{H}\left(g^{-1} x\right), & \text { para } x \in X-A, \text { onde } G_{x}=g H g^{-1} \\ f(x), & \text { para } x \in V .\end{cases}
$$

Essa homotopia é contínua porque para todo ponto em $x \in V-A$ temos que:

$$
g f_{t}^{H}\left(g^{-1} x\right)=g f\left(g^{-1} x\right)=g g^{-1} f(x)=f(x) .
$$

Segue que, $f_{1}(x)=g f_{1}^{H}\left(g^{-1} x\right)=x \Leftrightarrow f_{1}^{H}\left(g^{-1} x\right)=g^{-1} x$, para todo $x \in X-A$, onde $G_{x}=g H^{-1}$, o que garante a segunda parte do resultado.

O próximo resultado que queremos generalizar é o Teorema 2.1 de [24], um resultado referente a extensão de homotopias que preservam fibra. Para que sua demonstração fique clara devemos ser capazes de levantar caminhos de forma equivariante, contudo o melhor que temos é o Teorema XX.2.2 de [4] que trata apenas do caso não-equivariante. Por isso, antes de generalizar o Teorema 2.1 de [24] vamos generalizar o Teorema XX.2.2 de [4].

Proposição 3.3 Sejam $G$ um grupo finito, $E$ e $B$ G-espaços e $p: E \rightarrow B$ uma $G$ aplicação. Então $(E, p, B)$ é uma $G$-fibração (regular) se, e somente se, existe uma $G$ aplicação de levantamento (regular).

Prova da Proposição 3.3: Primeiramente vamos supor que $(E, p, B)$ possui uma $G$-aplicação de levantamento $\Lambda: \Omega_{p} \rightarrow E^{I}$. Sejam $X$ um $G$-espaço, $f: X \rightarrow E$ uma $G$-aplicação e $F: Z \times I \rightarrow B$ uma $G$-homotopia tais que $p \circ f(x)=F(x, 0)$, para todo 
$x \in X$ então definimos $\bar{F}(x, t)=\Lambda(f(x), F(x, \bullet))(t)$, para todo $(x, t) \in X \times I$, onde $F(x, \bullet): I \rightarrow X$ é o caminho dado por $F(x, \bullet)(t)=F(x, t)$. Segue que

$$
p \circ \bar{F}(x, t)=p \circ \Lambda(f(x), F(x, \bullet))(t)=F(x, \bullet)(t)=F(x, t),
$$

e $\bar{F}(x, 0)=\Lambda(f(x), F(x, \bullet))(0)=f(x)$, para todo $x \in X$. Além disso, se $\Lambda$ é regular e $x \in X$ for tal que $F(x, t)=F(x, 0)$ para todo $t \in I$ então $F(x, \bullet)$ é um caminho constante em $p \circ f(x)$. Segue que para todo $t \in I$ temos que

$$
\bar{F}(x, t)=\Lambda(f(x), \underbrace{F(x, \bullet)}_{=p \circ f(x)})(t)=\Lambda(f(x), p \circ f(x))(t)=f(x) .
$$

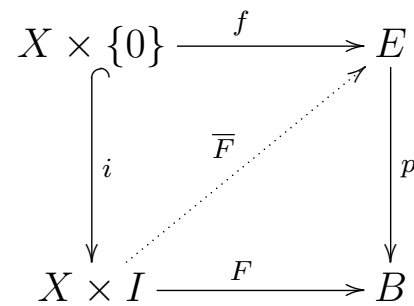

Agora vamos supor que $(E, p, B)$ é uma $G$-fibração. Sejam $f: \Omega_{p} \rightarrow E$ dada por $f(e, \alpha)=e$ a projeção (equivariante) na primeira coordenada e $F: \Omega_{p} \times I \rightarrow B$ dada por $F((e, \alpha), t)=\alpha(t)$. Segue que $p \circ f(x)=F(x, 0)$, para todo $(e, \alpha) \in \Omega_{p}$. Logo, existe $\bar{F}: \Omega_{p} \times I \rightarrow E$ tal que $p \circ \bar{F}=F$ e $\bar{F}((e, \alpha), 0)=f(e, \alpha)$, para todo $(e, \alpha) \in \Omega_{p}$.

Definimos $\Lambda: \Omega_{p} \rightarrow E^{I}$ dado por $\Lambda(e, \alpha)(t)=\bar{F}((e, \alpha), t)$, então:

$$
\begin{gathered}
\Lambda(e, \alpha)(0)=\bar{F}((e, \alpha), 0)=f(e, \alpha)=e ; \\
p \circ \Lambda(e, \alpha)(t)=p \circ \bar{F}((e, \alpha), t)=F((e, \alpha), t)=\alpha(t) ; \\
\Lambda(e, p(e))(t)=\bar{F}((e, p(e)), t)=e(t)=e .
\end{gathered}
$$

Como dissemos, queremos generalizar o Teorema 2.1 de [24]. Para tanto, fizemos a Proposição 3.3 para relacionar levantamentos de caminhos com fibrações, de forma equivariante. Agora faremos uma generalização do Corolário 2.7.14 de [2] que relaciona $G$-fibrados com $G$-fibrações e a usaremos para generalizar o Teorema 2.1 de [24] e na generalização dos resultados de [19].

Proposição B.1: Sejam $G$ um grupo finito e $\mathfrak{F}=(X, p, B, Y)$ um $G$-fibrado com espaço base $B$ Hausdorff e paracompacto, então $(X, p, B)$ é uma $G$-fibração regular.

Prova da Proposição B.1: Apêndice B. 
Antes de generalizar o Teorema 2.1 de [24] vamos considerar alguns objetos. Dado um espaço topológico $E$ tomemos $\alpha \in E^{I}$ e $t \in I$ e definimos $\alpha_{t} \in E^{I}$ sendo o caminho dado por $\alpha_{t}(s)=\alpha((1-s) t)$, para todo $s \in I$. Observamos que se $x$ for um ponto de $E$ e $\alpha$ um caminho em $B$ tais que $(x, \alpha) \in \Omega_{p}$ então podemos definir $y=\Lambda(x, \alpha)(1) \mathrm{e}$ $\alpha^{*}(t)=\alpha(1-t)$, onde $t \in I$ e $\Lambda$ é a aplicação de levantamento. Dessa forma, temos que:

$$
\Lambda(x, \alpha)(t)=\Lambda\left(y, \alpha^{*}\right)(1-t)=\Lambda\left(\Lambda(x, \alpha)(1), \alpha^{*}\right)(1-t),
$$

para todo $t \in I$, em particular $x=\Lambda(x, \alpha)(0)=\Lambda\left(\Lambda(x, \alpha)(1), \alpha^{*}\right)(1)$.

Proposição 3.4 Sejam $G$ um grupo finito, $\mathfrak{F}=(E, p, B)$ um $G$-fibrado , $(X, A)$ uma par de G-espaços métricos com A fechado em $X$ e invariante pela ação de $G$. Seja $\bar{H}:(X \times\{0\}) \cup(A \times I) \rightarrow E$ uma $G$-aplicação tal que $p \circ \bar{H}(x, 0)=p \circ \bar{H}(x, t)$ para todo $(x, t) \in A \times I$.

Então, se E for um $G$-ANR então $\bar{H}$ pode ser estendida para uma G-homotopia $H$ : $X \times I \rightarrow E$ tal que $p \circ H(x, 0)=p \circ H(x, t)$ para todo $(x, t) \in X \times I$.

Prova da Proposição 3.4: Com a Proposição 9.3 de [22] estendemos $\bar{H}$ para uma $G$-homotopia $H^{\prime}: X \times I \rightarrow E$. Notemos que $H^{\prime}$ pode ser visto como uma $G$-aplicação $X \rightarrow E^{I}$ dada por:

$$
\begin{aligned}
& H^{\prime}: X \rightarrow E^{I} \\
& x \mapsto H^{\prime}(x, \bullet): I \rightarrow E \\
& t \mapsto H^{\prime}(x, t) \text {. }
\end{aligned}
$$

Daí, definimos a $G$-extensão de $\bar{H}$ por $H(x, t)=\Lambda\left(H^{\prime}(x, t), p\left(H^{\prime}(x, \bullet)\right)_{t}\right)(1)$, onde $p\left(H^{\prime}(x, \bullet)\right)_{t}$ é o caminho definido por $p\left(H^{\prime}(x, \bullet)\right)_{t}(s)=p\left(H^{\prime}(x,(1-s) t)\right)$ e $\lambda$ é uma $G-$ aplicação de levantamento regular.

Notemos que:

$H(x, 0)=\Lambda\left(H^{\prime}(x, 0), p\left(H^{\prime}(x, \bullet)\right)_{0}\right)(1)=\Lambda\left(H^{\prime}(x, 0), p\left(H^{\prime}(x, 0)\right)\right)(1)=H^{\prime}(x, 0)=\bar{H}(x, 0)$.

Para todo $x \in A$ temos que $p\left(\left(H^{\prime}(x, \bullet)\right)_{t}\right)(s)=p(\bar{H}(x, 0))$, assim:

$$
H(x, t)=\Lambda\left(H^{\prime}(x, t), p\left(H^{\prime}(x, \bullet)\right)_{t}\right)(1)=\Lambda\left(\bar{H}(x, t), p \circ \bar{H}(x, \bullet)_{t}\right)(1)=\bar{H}(x, t) .
$$

Por fim, para todo $x \in X$ e $t \in I$ temos $p(H(x, t))=p\left(H^{\prime}(x, \bullet)_{t}\right)(1)=p(H(x, 0))=$ $p(H(x, 0))$.

O próximo resultado que faremos relaciona $G$-fibrações com base contrátil a $G$-fibração trivial. Essa é uma versão equivariante do Teorema XX.2.7 de [4]. Para entender o enunciado proposto devemos perceber que se $G$ é um grupo finito e $B$ é um $G$-espaço contrátil que se contrai para um ponto $b_{0} \in B$, para que a contração seja equivariante é necessário 
que $G_{b_{0}}=G$. Nesse sentido, quando dissermos que $B$ se contrai equivariantemente em $b_{0}$ teremos que $G_{b_{0}}=G$.

Contudo, podemos pensar em uma outra forma de generalizar as contrações para o contexto equivariante. Digamos que $G$ possui $n$ elementos e que $B$ possui $n$ componentes conexas, a saber $B=\bigcup_{i=1}^{n} B_{i}$. Seja $b_{0} \in B$ um ponto de $B$ tal que para cada $i=$ $1, \ldots, n$ existe $g_{i} \in G$ tal que $g_{i} b_{0} \in B_{i}$. Então podemos definir que $B$ se contrai equivariantemente em $G\left\{b_{0}\right\}$ e isso significa que cada componente conexa $B_{i}$ de $B$ se contrai em $g_{i} b_{0}$.

Proposição 3.5 Sejam $G$ um grupo finito, $(X, p, B)$ uma $G$-fibração regular e $b_{0} \in B$ um ponto tal que $B$ se contrai equivariantemente em $b_{0}$ ou $B$ se contrai equivariantemente em $G\left\{b_{0}\right\}$. Então $(X, p, B)$ é $G$-equivalente a $G$-fibração trivial $\left(B \times p^{-1}\left(\left\{b_{0}\right\}\right), q, B\right)$ para o caso em que $B$ se contrai equivariantemente em $b_{0}$ ou a $\left(B \times G p^{-1}\left(\left\{b_{0}\right\}\right), q, B\right)$ para o caso em que $B$ se contrai equivariantemente em $G\left\{b_{0}\right\}$.

Prova da Proposição 3.5: Consideremos o caso em que $B$ se contrai equivariantemente em $b_{0}$. Seja $\phi: B \times I \rightarrow B$ a contração de $B$ para o ponto $b_{0} \in B$, onde $\phi(b, 0)=b$ e $\phi(b, 1)=b_{0}$ para todo $b \in B$. Para cada $(b, s) \in B \times I$ definimos os caminhos $\alpha_{(b, s)}: I \rightarrow B$ dado por $\alpha_{(b, s)}(t)=\phi(b, s t)$, e $\alpha_{(b, s)}^{*}: I \rightarrow B$ dado por $\alpha_{(b, s)}^{*}(t)=\phi(b, s(1-t))$.

Daí, definimos as $G$-aplicações:

$$
\begin{array}{cccc}
f: & X & & B \times p^{-1}\left(\left\{b_{0}\right\}\right) \\
& x & \mapsto & \left(p(x), \Lambda\left(x, \alpha_{(p(x), 1)}\right)(1)\right), \\
h: B \times p^{-1}\left(\left\{b_{0}\right\}\right) & \rightarrow & X \\
(b, x) & & & \Lambda\left(x, \alpha_{(b, 1)}^{*}\right)(1),
\end{array}
$$

onde $\Lambda: \Omega_{p} \rightarrow E^{I}$ é a $G$-aplicação de levantamento regular. É importante observar que $f$ e $h$ são equivariantes porque dado $g \in G$ temos que $\alpha_{(g b, s)}(t)=\phi(g b, s t)=g \phi(b, s t)=$ $g \alpha_{(b, s)}(t)$, analogamente, $\alpha_{(g b, s)}^{*}(t)=g \alpha_{(b, s)}^{*}(t)$. A $G$-homotopia entre $h \circ f$ e $I d_{X}$ é dada por:

$$
\begin{array}{ccc}
H: X \times I & \rightarrow & X \\
(x, t) & \mapsto & \Lambda\left(\Lambda\left(x, \alpha_{(p(x), t)}\right)(1), \alpha_{(p(x), t)}^{*}\right)(1) .
\end{array}
$$

Observamos que $f \circ h=I d_{B \times p^{-1}\left(\left\{b_{0}\right\}\right)}$. De fato,

$$
\begin{aligned}
f \circ h(b, x) & =f\left(\Lambda\left(x, \alpha_{(b, 1)}^{*}\right)(1)\right) \\
& =(\underbrace{p\left(\Lambda\left(x, \alpha_{(b, 1)}^{*}\right)(1)\right)}_{=b}, \Lambda\left(\Lambda\left(x, \alpha_{(b, 1)}^{*}\right)(1), \alpha_{(\underbrace{(\underbrace{}_{(b, 1)}(1))}_{=b}, 1)}(1)\right)) \\
& =(b, \underbrace{\Lambda\left(\Lambda\left(x, \alpha_{(b, 1)}^{*}\right)(1), \alpha_{(b, 1)}(1)\right)}_{=x}) \\
& =(b, x) .
\end{aligned}
$$

A única diferença entre a prova do caso anterior e o caso " $B$ se contrai equivariantemente em $G\left\{b_{0}\right\}$ " é que a contração $\phi: B \times I \rightarrow B$ do primeiro se contrai no ponto $b_{0} \in B$ e a contração do segundo se contrai na órbita do ponto $b_{0} \in B$. 
Lembramos que dados um grupo de Lie compacto $G$, um $G$-fibrado $\mathfrak{F}=(X, p, B, Y)$, uma $G$-aplicação $f: X \rightarrow X$ que preserva fibra com relação a $\mathfrak{F}$ e uma $G$-homotopia $H: X \times I \rightarrow X$ que preserva fibra com relação a $\mathfrak{F}$, temos que $f$ induz uma $G$-aplicação $\bar{f}: B \rightarrow B$ tal que $\bar{f} \circ p=p \circ f$ e que $H$ induz uma $G$-homotopia $\bar{H}: B \times I \rightarrow B$ tal que $\bar{H}(p(x), t)=p \circ H(x, t)$, para todo $(x, t) \in X \times I$. O próximo resultado é uma generalização do Lema 2.1 de [19].

Proposição 3.6 Sejam G-um grupo de Lie compacto, $\mathfrak{F}=(X, p, B ; Y)$ um G-fibrado onde $X$ e $B$ são $G$-variedades conexas e compactas, $f: X \rightarrow X$ uma $G$-aplicação que preserva fibra e $A$ um subespaço invariante de $X$ tal que:

$(C 1)_{\mathfrak{F}}$ existe uma $G$-homotopia parcial que preserva fibra $H_{A}:(X \times\{0\}) \cup(A \times I) \rightarrow X$ tal que $H_{A}(\bullet, 0)=f$ e $H_{A}(\bullet, 1)=i: A \hookrightarrow X($ inclusão);

$(C 2)_{\mathfrak{F}}$ sempre que $W K$ for finito, toda $W K$-classe de pontos fixos essencial $F$ de $f^{K}$ está conectada a $A^{K}$ por um caminho $\alpha: I \rightarrow X^{K}$ tal que $\alpha(0) \in F, \alpha(1) \in A^{K} e\{\alpha(t)\}$ é homotópica à $\left\{f^{K} \circ \alpha\right\} *\left\{H_{A}^{K}(\alpha(1), t)\right\}$ com pontos extremos fixados.

Então, para toda $W K$-classe de pontos fixos essencial $F$ de $f^{K}$ existe um caminho $\bar{\alpha}: I \rightarrow$ $B^{K} \operatorname{com} \bar{\alpha}(0) \in p(F)^{K}, \bar{\alpha}(1) \in p(A)^{K} e\{\bar{\alpha}(t)\}$ é homotópica à $\left\{\bar{f}^{K} \circ \bar{\alpha}(t)\right\} *\left\{\bar{H}_{A}^{K}\left(p^{K} \circ\right.\right.$ $\alpha(1), t)\}$, com pontos extremos fixados, onde $H_{A}$ é a G-homotopia parcial dada em $(C 1)_{\mathfrak{F}}$.

Prova da Proposição 3.6: Seja $F$ uma $W K$-classe de pontos fixos essencial de $f^{K}$. Por hipótese temos um caminho $\alpha: I \rightarrow X^{K} \operatorname{com} \alpha(0) \in F, \alpha(1) \in A^{K}$ e $\{\alpha(t)\}$ é homotópica à $\left\{f^{K} \circ \alpha(t)\right\} *\left\{H_{A}^{K}(\alpha(1), t)\right\}$, com pontos extremos fixados.

Como $f^{K}$ e $H_{A}^{K}$ são ambas $W K$-aplicações que preservam fibra temos que $\left\{p^{K} \circ \alpha(t)\right\}$ é homotópica à

$$
\left\{p^{K} \circ f^{K} \circ \alpha(t)\right\} *\left\{p^{K} \circ H_{A}^{K}(\alpha(1), t)\right\}=\left\{\bar{f}^{K} \circ p^{K} \circ \alpha(t)\right\} *\left\{\bar{H}_{A}^{K}\left(p^{K} \circ \alpha(1), t\right)\right\} .
$$

Daí, definimos $\bar{\alpha}=p^{K} \circ \alpha$ e obtemos um caminho $\bar{\alpha}: I \rightarrow B^{K} \operatorname{com} \bar{\alpha}(0) \in p(F)^{K}$, $\bar{\alpha}(1) \in p(A)^{K}$ e $\{\bar{\alpha}(t)\}$ é homotópica à $\left\{\bar{f}^{K} \circ \bar{\alpha}(t)\right\} *\left\{\bar{H}_{A}^{K}\left(p^{K} \circ \alpha(1), t\right)\right\}$, com pontos extremos fixados.

A próxima observação que faremos está relacionada com a proposição anterior e foi retirada do Teorema 4.2 de [9].

Proposição 3.7 Sejam $G$ um grupo de Lie compacto, $X$ um $G$-ANR e $f: X \rightarrow X$ uma $G$-aplicação. Se $A$ for um subconjunto fechado e invariante de $X$ tal que $A \subset F i x(f) e$ $A^{K}$ intercepta toda $W K$-classe de pontos fixos essencial $F$ de $f^{K}$, onde $(K) \in I s o(X) e$ $W K$ é finito, então as seguintes condições são verdadeiras: 
(C1) existe uma G-homotopia parcial que preserva fibra $H_{A}:(X \times\{0\}) \cup(A \times I) \rightarrow X$ tal que $H_{A}(\bullet, 0)=f$ e $H_{A}(\bullet, 1)=i: A \hookrightarrow X($ inclusão $)$;

(C2) sempre que $W K$ for finito, toda $W K$-classe de pontos fixos essencial $F$ de $f^{K}$ está conectada a $A^{K}$ por um caminho $\gamma: I \rightarrow X^{K}$ tal que $\gamma(0) \in F, \gamma(1) \in A^{K} e\{\gamma(t)\}$ é homotópica à $\left\{f^{K} \circ \gamma\right\} *\left\{H_{A}^{K}(\gamma(1), t)\right\}$ com pontos extremos fixados, onde $H_{A} e^{\prime}$ a homotopia.

Prova da Proposição 3.7: A homotopia parcial dada por:

$$
\begin{aligned}
H_{A}:(X \times\{0\}) \cup(A \times I) & \rightarrow \\
(x, t) & \mapsto\left\{\begin{array}{cl}
x & \operatorname{para}(x, t) \in A \times I \\
f(x) & \operatorname{para}(x, t) \in X \times\{0\},
\end{array}\right.
\end{aligned}
$$

é a homotopia que satisfaz $(C 1)$, e dada uma $W K$-classe de pontos fixos essencial $F$ de $f^{K}$ seja $x \in A^{K} \cap F \neq \emptyset$, daí, definimos $\gamma: I \rightarrow X^{K}$ por $\gamma(t)=x$, para todo $t \in I$, que satisfaz $(C 2)$. 


\subsection{Realização de um Conjunto como Conjunto de Pon- tos Fixos de uma dada $G$-Aplicação que Preserva Fibra}

Generalizamos nessa seção o Teorema 4.1 de [19] seguindo os passos usados na versão original.

No próximo resultado estamos supondo que $I s o(B)=\left\{\left(K_{i}\right)\right\}_{i=1}^{n}$ está munido de uma ordem admissível, que $p(A)_{i}=B_{i} \cap p(A)$, para $i=1, \ldots, n$, onde $B_{1} \subset \cdots \subset B_{n}=B$ é a filtração de $B$ e que $T=\left\{i \in\{1, \ldots, n\} ;\left(K_{i}\right) \in I s o(B)\right.$ e W $K_{i}$ é finito $\}$.

Lema 3.8 Sejam $G$ um grupo de Lie compacto, $\mathfrak{F}=(X, p, B, Y)$ um $G$-fibrado onde $X$ e $B$ são G-variedades suaves, compactas e para $W K$ finito temos que $\operatorname{dim}\left(B^{K}\right) \geq 3$, $\operatorname{dim}\left(B^{K}\right)-\operatorname{dim}\left(B^{K}-B_{K}\right) \geq 2$, para todo $(K) \in I s o(B)$.

Sejam $A$ um $G$-subconjunto não vazio de $X$ tal que $p(A)$ é um $G$-subconjunto fechado $e$ localmente contrátil de $B$ tal que $p^{K}\left(A^{K}\right)$ é contornável em $B^{K}$, para todo $(K) \in I s o(B)$. Se $f: X \rightarrow X$ for uma $G$-aplicação que preserva fibra tal que :

$(C 1)_{\mathfrak{F}}$ existe uma G-homotopia parcial que preserva fibra $H_{A}:(X \times\{0\}) \cup(A \times I) \rightarrow X$ tal que $H_{A}(\bullet, 0)=f$ e $H_{A}(\bullet, 1)=i: A \hookrightarrow X($ inclusão $)$;

$(C 2)_{\mathfrak{F}}$ sempre que $W K$ for finito, toda $W K$-classe de pontos fixos essencial $F$ de $f^{K}$ está conectada a $A^{K}$ por um caminho $\gamma: I \rightarrow X^{K}$ tal que $\gamma(0) \in F, \gamma(1) \in A^{K} e\{\gamma(t)\}$ é homotópica à $\left\{f^{K} \circ \gamma\right\} *\left\{H_{A}^{K}(\gamma(1), t)\right\}$ relativo aos extremos.

Então existe uma G-homotopia $\bar{H}: B \times I \rightarrow B$ que estende a $G$-aplicação $\bar{H}_{A}$ : $p(A) \times I \rightarrow B$ induzida por $H_{A}$ dada em $(C 1)_{\mathfrak{F}}$ tal que $\bar{H}(\bullet, 0): B \rightarrow B$ é a induzida de $f e$

$$
\operatorname{Fix}(\bar{H}(\bullet, 1))=p(A) \cup\left(\bigcup_{i \in T}\left(G\left\{b_{i, 1}\right\} \cup \cdots \cup G\left\{b_{i, m_{i}}\right\}\right)\right)
$$

onde:

1. $K_{i}$ está na mesma classe de conjugação da isotropia de $b_{i, j}$, onde $i \in T$ e $j=$ $1, \ldots, m_{i}$

2. $W K_{i}\left\{b_{i, j}\right\}$ é uma $W K_{i}$-classe de pontos fixos essencial de $\bar{H}^{K_{i}}(\bullet, 1)$, para $j=$ $1, \ldots, m_{i}$;

3. não existe $W K$-classe de pontos fixos essencial $F$ de $f^{K}$ tal que $p^{K}(F)=W K\left\{b_{i, j}\right\}$, para todo $(K) \in I s o(X)$ com WK finito e todos $1 \leq i \leq n$ e $1 \leq j \leq m_{i}$.

Prova do Lema 3.8: Como $p(A)$ é um G-subconjunto fechado de $B$ temos que a $G$-aplicação que preserva fibra $H_{A}: A \times I \rightarrow X$ dada em $C 1_{\mathfrak{F}}$ induz uma $G$-aplicação $\bar{H}_{A}: p(A) \times I \rightarrow B$ tal que $\bar{H}_{A}(\bullet, 0)=\bar{f}$ e $\bar{H}_{A}(\bullet, 1)=i_{p(A)}: p(A) \hookrightarrow B$ a inclusão. 
Agora faremos uma construção em $B$ referente a $\bar{H}_{A}$ análoga a que fizemos na demonstração do Teorema 2.10. Basicamente, $(C 2)$ é uma hipótese que ajuda a eliminar pontos fixos residuais, mas não a temos aqui. Por isso, faremos aqui a mesma construção que fizemos lá a menos de $(C 2)$.

Suponhamos que estendemos $\bar{H}_{A}: p(A) \times I \rightarrow B$ para uma $G$-aplicação $\bar{H}_{i-1, A}$ : $\left(p(A) \cup B_{i-1}\right) \times I \rightarrow B$. Daí, usamos a Proposição 9.3 de [22] para estender $\bar{H}_{i-1, A}$ para uma $G$-aplicação $\bar{H}_{i, 1}:\left(B_{i} \cup p(A)\right) \times I \rightarrow B$.

Se $W K_{i}$ for um grupo de Lie de dimensão positiva então existem $V$ uma vizinhança invariante de $p(A)_{i} \cup B_{i-1}$ em $B_{i}$ que se retrai equivariantemente em $B_{i-1} \cup p(A)_{i}$ (Proposição $2.2)$ e uma $W K_{i}$-homotopia $\bar{H}_{i, 2}:\left(B_{i} \cup p(A)\right)^{K_{i}} \times I \rightarrow B^{K_{i}}$ relativa a $\left(B_{i-1} \cup p(A)\right)^{K_{i}}$ entre $\bar{H}_{i, 1}^{K_{i}}(\bullet, 1)$ e a aplicação $\bar{H}_{i, 2}(\bullet, 1)$ que não possui pontos fixos em $B_{i}^{K_{i}}-\left(B_{i-1} \cup p(A)\right)^{K_{i}}$ (Lema 3.3 de [18]).

Daí, com o Lema 2.1 de [12] estendemos a $W K_{i}$-homotopia para uma $G$-homotopia $\bar{H}_{i}:\left(B_{i} \cup p(A)\right) \times I \rightarrow B$ relativa a vizinhança $V$ de modo que $\bar{H}_{i}(\bullet, 1)$ não possui pontos fixos em $B_{i}-\left(B_{i-1} \cup p(A)\right)$ e $p(A) \subset \operatorname{Fix}\left(\vec{H}_{i}(\bullet, 1)\right)$.

Se $W K_{i}$ for um grupo finito então existem uma $W K_{i}$-triangulação de $B_{i}^{K_{i}}$ na qual $B_{i-1}^{K_{i}}$ é um $W K_{i}$-subpoliedro de $B_{i}^{K_{i}}$ e $S t\left(p\left(A_{i}^{K_{i}}\right)\right)$ é contornável em $B_{i}^{K_{i}}$ e uma vizinhança invariante $V$ de $S t\left(p\left(A_{i}\right)\right) \cup B_{i-1}$ em $B_{i}$ que se retrai $G$-equivariantemente em $\operatorname{St}\left(p\left(A_{i}\right)\right) \cup$ $B_{i-1}$ (Proposições 2.2, 2.3 e 2.4).

Segue do Lema 3.1 de [18] que existe uma $W K_{i}$-aplicação $\bar{H}_{i, 2}:\left(B_{i} \cup p(A)\right)^{K_{i}} \times I \rightarrow B_{i}^{K_{i}}$ relativa a $V^{K_{i}}$, entre $\bar{H}_{i, 1}^{K_{i}}(\bullet, 1)$ e uma aplicação $\bar{H}_{i, 2}(\bullet, 1)$ tal que:

1. $p(A)^{K_{i}} \subset \operatorname{Fix}\left(\bar{H}_{i, 2}(\bullet, 1)\right)$;

2. $\left(U-p(A)^{K_{i}}\right) \cap \operatorname{Fix}\left(\bar{H}_{i, 2}(\bullet, 1)\right)=\emptyset$ para alguma uma vizinhança $W K_{i}$-invariante $U$ de $\operatorname{St}\left(p(A)_{i}^{K_{i}}\right)$ em $B_{i}^{K_{i}}$;

3. $\bar{H}_{i, 2}(\bullet, 1)$ possui uma quantidade finita de pontos fixos em $B_{i}^{K_{i}}-V^{K_{i}}$ todos contidos no interior de simplexos maximais da triangularização de $B_{i}^{K_{i}}$.

Pelo Procedimento 2.8 podemos colapsar todas as $W K_{i}$-órbitas de pontos fixos de $\bar{H}_{i, 2}(\bullet, 1)$ que se encontram em $B_{i}^{K_{i}}-V^{K_{i}}$ e que são $G$-Nielsen equivalentes entre si ou a alguma $W K_{i}$-órbita de pontos fixos que se encontra em $\partial\left(p(A)_{i}^{K_{i}}\right)$ na $W K_{i}$-órbita de pontos fixos de $\partial\left(p(A)_{i}^{K_{i}}\right)$. Daí, usamos o Teorema VIII.B.4 de [1] para removemos todas as $W K_{i}$-órbitas de pontos fixos $F=W K_{i}\{x\}$ de índice 0 de $\bar{H}_{i, 3}(\bullet, 1)$ que não interceptam $p(A)_{i}^{K_{i}}$.

Dessa forma, obtemos uma $W K_{i}$-homotopia $\bar{H}_{i}: B_{i}^{K_{i}} \times I \rightarrow B_{i}^{K_{i}}$ entre $\bar{H}_{i, 3}(\bullet, 1)$ e a $W K_{i}$-aplicação $\bar{h}=\bar{H}_{i}(\bullet, 1)$ tal que:

1. $p(A)_{i}^{K_{i}} \subset \operatorname{Fix}(\bar{h})$;

2. $\bar{h}$ possui uma quantidade finita de pontos fixos em $B_{i}^{K_{i}}-V^{K_{i}}$; 
3. dada uma $W K_{i}$-classe de pontos fixos $F$ de $\bar{h}$ tal que $F \cap p(A)_{i}^{K_{i}}=\emptyset$ então $F=$ $W K_{i}\{x\} \operatorname{com} x \in B_{i}^{K_{i}}-V^{K_{i}}$ e $F$ é uma $W K_{i}$-classe de pontos fixos essencial de $\bar{h}$.

Com a Proposição 3.2 estendemos a $W K_{i}$-homotopia para uma $G$-homotopia $\bar{H}_{i}$ : $\left(B_{i} \cup p(A)\right) \times I \rightarrow B$ relativa a vizinhança $V$ de modo que

$$
\operatorname{Fix}\left(\bar{H}_{i}(\bullet, 1)\right)=p(A) \cup\left(\bigcup_{j \in T, j \leq i}\left(G\left\{b_{j, 1}\right\} \cup \cdots \cup G\left\{b_{j, m_{j}}\right\}\right)\right)
$$

e $W K_{i}\left\{b_{i, l}\right\}$ é uma $W K_{i}$-classe de pontos fixos essencial de $\bar{H}_{i}^{K_{i}}(\bullet, 1)$, para $1 \leq l \leq m_{i}$.

Uma outra característica de $G\left\{b_{i, l}\right\}$ é que se $p^{K}(F)=W K\left\{b_{i, l}\right\}$ para alguma $W K$ classe de pontos fixos essencial $F$ de $f^{K}$, onde $(K) \in I s o(X)$ com $W K$ finito, teríamos um caminho $\bar{\alpha}$, dado na Proposição 3.6, com a seguinte propriedade:

$$
\{\bar{\alpha}\} \sim\left\{\bar{f}^{K} \circ \bar{\alpha}\right\} *\left\{\bar{H}_{A}^{K}(\bar{\alpha}(1), t)\right\} \sim\left\{\bar{H}^{K}(\bar{\alpha}(t), t)\right\} .
$$

Portanto, $\bar{\alpha}(1)=g b_{i, l}$, para algum $g \in W K$ e $\bar{\alpha}(1) \in p^{K}(A)$, o que é um absurdo pois $b_{i, l} \notin p^{K}(A)$ e $p^{K}(A)$ é $W K$-invariante.

Daí, usamos os casos 1 e 2 indutivamente para estender a $G$-aplicação $\bar{H}_{A}: p(A) \times I \rightarrow$ $B$ para uma $G$-homotopia $\bar{H}: B \times I \rightarrow B$ com as propriedades descritas nos casos 1 e 2 .

Lema 3.9 Sejam $G$ um grupo finito, $\mathfrak{F}=(X, p, B, Y)$ um $G$-fibrado onde $X$ e $B$ são $G$-variedades suaves, compactas e $\operatorname{dim}\left(B^{K}\right) \geq 3, \operatorname{dim}\left(B^{K}\right)-\operatorname{dim}\left(B^{K}-B_{K}\right) \geq 2$, para todo $(K) \in I$ so $(B)$.

Sejam $A$ um $G$-subconjunto não vazio, fechado e localmente contrátil $X$ tal que $p(A)$ é um $G$-subconjunto fechado de $B$ e $p^{K}\left(A^{K}\right)$ é contornável em $B^{K}$, para todo $(K) \in I s o(B)$. Se $f: X \rightarrow X$ for uma $G$-aplicação que preserva fibra tal que:

$(C 1)_{\mathfrak{F}}$ existe uma $G$-homotopia parcial que preserva fibra $H_{A}:(X \times\{0\}) \cup(A \times I) \rightarrow X$ tal que $H_{A}(\bullet, 0)=f$ e $H_{A}(\bullet, 1)=i: A \hookrightarrow X($ inclusão $)$;

$(C 2)_{\mathfrak{F}}$ sempre que $W K$ for finito, toda $W K$-classe de pontos fixos essencial $F$ de $f^{K}$ está conectada a $A^{K}$ por um caminho $\gamma: I \rightarrow X^{K}$ tal que $\gamma(0) \in F, \gamma(1) \in A^{K}$ e $\{\gamma(t)\}$ é homotópica ̀̀ $\left\{f^{K} \circ \gamma\right\} *\left\{H_{A}^{K}(\gamma(1), t)\right\}$ com pontos extremos fixados.

Então existe uma $G$-aplicação que preserva fibra $h: X \rightarrow X$ G-homotópica a $f$ por uma homotopia que preserva fibra tal que $A \subset F i x(h) \subset p^{-1}(p(A))$ e Fix $(\bar{h}) \cap(B-p(A))$ é finito.

Prova do Lema 3.9: Tomemos uma ordem admissível para $I s o(B)=\left\{\left(K_{i}\right)\right\}_{i=1}^{n}$. Com o Lema 3.8 criamos uma $G$-homotopia $\bar{H}: B \times I \rightarrow B$ que estende a $G$-aplicação $\bar{H}_{A}: p(A) \times I \rightarrow B$ induzida por $H_{A}$ dada em $C 1_{\mathfrak{F}}$ tal que $\bar{H}(\bullet, 0): B \rightarrow B$ é a induzida de $f$ e $F i x(\bar{H}(\bullet, 1))=p(A) \cup\left(\cup_{1 \leq i \leq n}\left(G\left\{b_{i, 1}\right\} \cup \cdots \cup G\left\{b_{i, m_{i}}\right\}\right)\right)$, onde: 
1. $K_{i}$ está na mesma classe de conjugação da isotropia de $b_{i, j}$, onde $i=1, \ldots, n$ e $j=1, \ldots, m_{i}$;

2. $W K_{i}\left\{b_{i, j}\right\}$ é uma $W K_{i}$-classe de pontos fixos essencial de $\bar{H}^{K_{i}}(\bullet, 1)$, para $j=$ $1, \ldots, m_{i}$;

3. não existe $W K$-classe de pontos fixos essencial $F$ de $f^{K}$ tal que $p^{K}(F)=W K\left\{b_{i, j}\right\}$, para todo $(K) \in I s o(X), 1 \leq i \leq n$ e $1 \leq j \leq m_{i}$.

Usaremos a notação $b_{i, j}$ somente quando for necessário e usaremos apenas um índice nos outros casos.

Denotaremos $\bar{h}=\bar{H}(\bullet, 1)$. Agora queremos provar que $f: X \rightarrow X$ é $G$-homotópica por uma homotopia que preserva fibra a uma aplicação $h: X \rightarrow X$ tal que Fix $(h) \subset$ $p^{-1}(p(A))$.

Com $\bar{H}$ definimos $H^{\prime}: X \times I \rightarrow B$ por $H^{\prime}(x, t)=\bar{H}(p(x), t)$. Observemos que $H^{\prime}(x, 0)=\bar{H}(p(x), 0)=\bar{f} \circ p(x)=p \circ f(x)$. Pela Proposição B.1 levantamos $H^{\prime} \mathrm{e}$ encontramos uma $G$-homotopia que preserva fibra $H_{1}: X \times I \rightarrow X$ tal que $f(x)=H_{1}(x, 0)$ e $h_{1}(x)=H_{1}(x, 1)$. Logo,

$$
F i x\left(h_{1}\right) \subset p^{-1}(F i x(\bar{h}))=p^{-1}\left(p(A) \cup G\left\{b_{1}\right\} \cup \cdots \cup G\left\{b_{l}\right\}\right) .
$$

Observamos que dado um ponto $a \in A$ temos que $p \circ h_{1}(a)=p(a)$, contudo, não podemos dizer que $h_{1}(a)=a$, mas podemos dizer que $h_{1}(a) \in p^{-1}(p(A))$. E assim, não sabemos se $A$ está em Fix $\left(h_{1}\right)$.

Para cada $G$-órbita $G\left\{b_{j}\right\}$ consideremos a restrição de $h_{1}$ para $G p^{-1}\left(b_{j}\right)=p^{-1}\left(G\left\{b_{j}\right\}\right)$ (denotaremos por $h_{1, b_{j}}$ ). Vamos mostrar que $h_{1, b_{j}}: G p^{-1}\left(b_{j}\right) \rightarrow G p^{-1}\left(b_{j}\right)$ não possui classes de pontos fixos essenciais.

De fato, seja $(K) \in I s o(X)$ e suponhamos que $h_{1, b_{j}}^{K}$ possui uma $W K$-classe de pontos fixos essencial $F$. Então, dado $x \in F$ temos que $W K p^{K}(x)=W K\left\{b_{j}\right\}$ é uma $W K$-classe de pontos fixos essencial de $\bar{h}^{K}$. Como $W K\{x\}$ está em uma $W K$-classe de pontos fixos de $h_{1}^{K}$, pois $x$ é um ponto fixo de $h_{1}^{K}$, segue que existe uma $W K$-classe de pontos fixos $Q$ de $h_{1}^{K}$ que contém $W K\{x\}$.

Observamos que $h_{1}^{K}$ é $W K$-homotópica a $f^{K}$, por uma homotopia que preserva fibra. Logo, existe alguma $W K$-classe de pontos fixos essencial $D$ de $f^{K} H_{1}^{K}$-relacionada com $Q$. Portanto, $p^{K}(D)$ está $\bar{H}^{K}$-relacionada com $W K\left\{b_{j}\right\}$, o que contradiz o Lema 3.8 a respeito dos pontos fixos de $\bar{h}$.

Consequentemente, $h_{1, b_{j}}$ não possui nenhuma classe de pontos fixos essencial, e assim, $h_{1, b_{j}}$ é $G$-homotópica, pela $G$-homotopia $H_{2, b_{j}}$ que preserva fibra, a uma $G$-aplicação $h_{2, b_{j}}: G p^{-1}\left(b_{j}\right) \rightarrow G p^{-1}\left(b_{j}\right)$ livre de pontos fixos.

Daí, conseguimos uma $G$-aplicação $\widetilde{H}_{2}:(X \times\{0\}) \cup\left(p^{-1}(F i x(\bar{h})) \times I\right) \rightarrow X$ definida por:

$$
\widetilde{H}_{2}(x, t)= \begin{cases}h_{1}(x) & \text { se } t=0 \text { ou se } x \in p^{-1}(p(A)) \\ H_{2, b_{j}}(x, t) & \text { se } x \in p^{-1}\left(G\left\{b_{j}\right\}\right)\end{cases}
$$


Então com a Proposição 3.4 estendemos $\widetilde{H}_{2}$ para uma $G$-aplicação $H_{2}: X \times I \rightarrow X$. Definimos $h_{2}=H_{2}(\bullet, 1): X \rightarrow X$. Essa extensão garante que $\bar{H}_{2}(\bullet, 1)=\bar{h}$.

Agora falta provar que $f$ é $G$-homotópica, por uma homotopia que preserva fibra, a uma aplicação $h: X \rightarrow X$ tal que $A \subset F i x(h) \subset p^{-1}(p(A))$.

Por $C 1_{\mathfrak{F}},\left.f\right|_{A}$ é $G$-homotópica por uma homotopia que preserva fibra a inclusão $i_{A}$, dessa forma $\left.h_{2}\right|_{A}$ também é $G$-homotópica por uma homotopia que preserva fibra a inclusão $i_{A}$. Denotaremos essa homotopia parcial por $\widetilde{H}_{A},\left.\operatorname{com} h_{2}\right|_{A}=\widetilde{H}_{A}(\bullet, 0)$ e $i_{A}=\widetilde{H}_{A}(\bullet, 1)$. Daí, definimos:

$$
\begin{aligned}
\widetilde{H}:(X \times\{0\}) \cup\left(\left(A \cup p^{-1}\left(G\left\{b_{1}, \ldots, b_{r}\right\}\right)\right) \times I\right) & \rightarrow X \\
(x, t) & \mapsto \begin{cases}h_{2}(x), & \text { se } t=0 ; \\
h_{2, b_{j}}(x), & \text { se } x \in G p^{-1}\left(b_{j}\right) ; \\
\widetilde{H}_{A}(x, t), & \text { se } x \in A .\end{cases}
\end{aligned}
$$

Usando novamente a Proposição 3.4 estendemos $\widetilde{H}$ para uma $G$-homotopia que preserva fibra $H: X \times I \rightarrow X$.

Uma consequência imediata do Lema 3.9 é que se $A=p^{-1}(p(A))$ então é possível encontrar uma $G$-aplicação que preserva fibra $h: X \rightarrow X G$-homotópica a $f$ por uma homotopia que preserva fibra tal que $F i x(h)=A$. Por outro lado, o Lema 3.1 diz que se existir uma $G$-aplicação que preserva fibra $h: X \rightarrow X G$-homotópica a $f$, por uma homotopia que preserva fibra, tal que $F i x(h)=A$ então as condições $C 1_{\mathfrak{F}}$ e $C 2_{\mathfrak{F}}$ são válidas.

Dessa forma, existe uma $G$-aplicação que preserva fibra $h: X \rightarrow X G$-homotópica a $f$ por uma homotopia que preserva fibra tal que $F i x(h)=A$ se, e somente se, as condições $C 1_{\mathfrak{F}}$ e $C 2_{\mathfrak{F}}$ forem válidas.

Para o próximo resultado devemos entender que se $f: X \rightarrow X$ é uma aplicação tal que $p \circ f=p$ então $f$ preserva fibra, pois se $p(x)=p(y)$ então

$$
p \circ f(x)=p(x)=p(y)=p \circ f(y) .
$$

Também usaremos que se um $G$-espaço $B$ se contrai equivariantemente em $b_{0}$ isso significa que $G_{b_{0}}=G$. Daí, $G=(G)=\left(K_{1}\right) \in I s o(B)$ e $W G=\{e\}$.

Lema 3.10 Sejam $G$ um grupo finito, $\left(\mathfrak{F}, \mathfrak{F}_{0}\right)=\left((X, A), p, B,\left(Y, Y_{0}\right)\right)$ um G-par fibrado, onde $X, B$ e $Y$ são $G$-variedades suaves e compactas, $B$ se contrai equivariantemente em $b_{0} e \operatorname{dim}\left(Y^{K}\right) \geq 3$ e $\operatorname{dim}\left(Y^{K}\right)-\operatorname{dim}\left(Y^{K}-Y_{K}\right) \geq 2$, para $(K) \in I s o(Y)$.

Sejam $Y_{0}$ um $G$-subconjunto fechado, localmente contrátil de $Y$ tal que $Y_{0}^{K}$ é contornável em $Y^{K}$, para todo $(K) \in I s o(Y), A$ um $G$-subconjunto fechado, localmente contrátil e não vazio de $X$ e $f: X \rightarrow X$ uma $G$-aplicação tal que $p \circ f=p, A \subset F i x(f), A^{K}$ intercepta todas as $W K$-classes de pontos fixos essenciais de $f_{b_{0}}^{K}: W K\left(p^{K}\right)^{-1}\left(b_{0}\right) \rightarrow$ $W K\left(p^{K}\right)^{-1}\left(b_{0}\right)$ para todo $(K) \in I s o(X)$. 
Seja $Z$ um fechado $G$-invariante que intercepta todas as componentes de $A$ tal que $(A, Z)$ é um $G$-par fibrado de $\mathfrak{F}_{0}$. Então existe uma $G$-aplicação $h: X \rightarrow X$ tal que $F i x(h)=Z$ e h é G-homotópica a f por uma homotopia que preserva fibra.

Prova do Lema 3.10: Pela Proposição $3.5(X, p, B)$ é $G$-equivalente a $G$-fibração trivial $(B \times Y, \pi, B)$, onde $\pi$ é a projeção em $B$. Assim, existe $\Phi: B \times Y \rightarrow X$ um $G$-homeomorfismo tal que $\Phi\left(B \times Y_{0}\right)=A$ e $p \circ \Phi=\pi$.

Definimos a $G$-aplicação $f^{*}=\Phi^{-1} \circ f \circ \Phi: B \times Y \rightarrow B \times Y$ e notemos que:

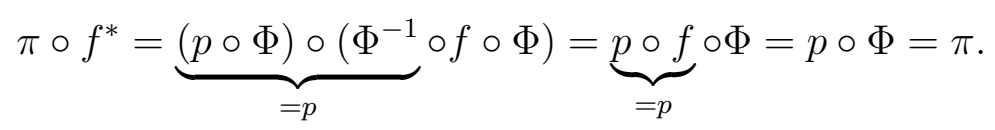

Dessa forma, podemos escrever $f^{*}(b, y)=\left(b, f_{b}^{*}(y)\right)$ e $g f^{*}(b, y)=\left(g b, f_{g b}^{*}(g y)\right)$, para todo $g \in G$.

$B$ se contrai equivariantemente em $b_{0}$, assim existe uma $G$-homotopia $D: B \times I \rightarrow B$ tal que para todo $b \in B$ temos que $D(b, 0)=b$ e $D(b, 1)=b_{0}$. Definimos a $G$-homotopia $U^{*}: B \times Y \times I \rightarrow B \times Y$ dada por:

$$
U^{*}(b, y, t)= \begin{cases}\left(b, f_{D(b, 2 t)}^{*}(y)\right), & \text { se } 0 \leq t \leq \frac{1}{2} \\ \left(b, f_{D\left(b_{0}, 2-2 t\right)}^{*}(y)\right), & \text { se } \frac{1}{2} \leq t \leq 1 .\end{cases}
$$

Assim, $U^{*}(b, y, 0)=\left(b, f_{b}^{*}(y)\right)=f^{*}(b, y)$ e $U^{*}(b, y, 1)=\left(b, f_{b_{0}}^{*}(y)\right)$ o que garante que $f^{*}$ é $G$-homotópica a $i d \times f_{b_{0}}^{*}$.

Lembrando que $\Phi\left(B \times Y_{0}\right)=A \subset F i x(f)$ temos que para todo $(b, y) \in B \times Y_{0}$ :

$$
f^{*}(b, y)=\Phi^{-1} \circ \underbrace{f \circ \underbrace{\Phi(b, y)}_{\in A}}_{=\Phi(b, y)}=\Phi^{-1} \circ \Phi(b, y)=(b, y) .
$$

Assim, $B \times Y_{0} \subset F i x\left(f^{*}\right)$ e $Y_{0} \subset F i x\left(f_{b}^{*}\right)$, pois $\left(b, f_{b}^{*}(y)\right)=f^{*}(b, y)=(b, y)$. Em particular, $Y_{0} \subset F i x\left(f_{b_{0}}^{*}\right)$.

Por hipótese, $A$ intercepta todas as $W K$-classes de pontos fixos essenciais de $f_{b_{0}}^{K}$ : $W K\left(p^{K}\right)^{-1}\left(b_{0}\right) \rightarrow W K\left(p^{K}\right)^{-1}\left(b_{0}\right)$. Daí, $A \cap W K\left(p^{K}\right)^{-1}\left(b_{0}\right)$ intercepta todas as $W K$ classes de pontos fixos essenciais de $f_{b_{0}}^{K}$. Lembramos que $\Phi^{-1}$ é um $G$-homeomorfismo e com isso estabelece uma correspondência biunívoca entre as $W K$-classes de pontos fixos de $f_{b_{0}}^{K}$ e $\left(f_{b_{0}}^{*}\right)^{K}$. Dessa forma, temos que $Y_{0}$ intercepta todas as $W K$-classes de pontos fixos essenciais de $\left(f_{b_{0}}^{*}\right)^{K}$, pois:

$$
\left(\Phi^{-1}\right)^{K}\left(A^{K} \cap W K\left(p^{K}\right)^{-1}\left(b_{0}\right)\right)=W K\left\{b_{0}\right\} \times Y_{0}^{K} .
$$

O $G$-par fibrado $\left((A, Z), p, B,\left(Y_{0}, \Omega\right)\right)$ é tal que o conjunto $\Omega$ intercepta todas as componentes conexas de $Y_{0}$, pois $Z$ intercepta todas as componentes conexa de $A$ por hipótese. 
Pela Proposição $3.7 Y_{0}$ satisfaz $(C 1)_{\mathfrak{F}}$ e $(C 2)_{\mathfrak{F}}$ para $f_{b_{0}}^{*}$. Segue do Teorema 2.10 que existe uma homotopia $V^{*}: Y \times I \rightarrow Y$ entre $f_{b_{0}}^{*}=V^{*}(\bullet, 0)$ e uma aplicação $V^{*}(\bullet, 1)=$ $g_{b_{0}}^{*}: Y \rightarrow Y$ tal que $\operatorname{Fix}\left(g_{b_{0}}^{*}\right)=\Omega$. Definimos a $G$-homotopia que preserva fibra $H^{*}$ : $B \times Y \times I \rightarrow B \times Y$ dada por:

$$
H^{*}(b, y, t)= \begin{cases}U^{*}(b, y, 2 t), & \text { se } 0 \leq t \leq \frac{1}{2} \\ \left(b, V^{*}(y, 2 t-1)\right), & \text { se } \frac{1}{2} \leq t \leq 1\end{cases}
$$

Assim, $f^{*}$ é $G$-homotópico a $i d \times g_{b_{0}}^{*}$ e $F i x\left(i d \times g_{b_{0}}^{*}\right)=B \times \Omega$.

Por fim, definimos a $G$-homotopia $H: X \times I \rightarrow X$ dada por $H(e, t)=\Phi \circ H^{*}\left(\Phi^{-1}(e), t\right)$. Assim, $H(e, 0)=f(e)$ e $\operatorname{Fix}(H(\bullet, 1))=\Phi(B \times \Omega)=Z$.

Teorema 3.11 Dados $G$ um grupo finito, $\mathfrak{F}=(X, p, B, Y)$ um $G$-fibrado onde $X, B$ e $Y$ são $G$-variedades suaves e compactas, $\operatorname{dim}\left(B^{K}\right) \geq 3, \operatorname{dim}\left(B^{K}\right)-\operatorname{dim}\left(B^{K}-B_{K}\right) \geq 2$, para $(K) \in I \operatorname{Iso}(B)$, e $\operatorname{dim}\left(Y^{K}\right) \geq 3$ e $\operatorname{dim}\left(Y^{K}\right)-\operatorname{dim}\left(Y^{K}-Y_{K}\right) \geq 2$, para $(K) \in I \operatorname{Iso}(Y)$.

Seja $A$ um $G$-subconjunto não vazio, fechado e localmente contrátil de $X$ tal que $(X, A)$ é um $G$-par de fibrados para $\mathfrak{F}, p(A)$ é um $G$-subconjunto fechado de $B$, cada componente $p(A)_{j}$ de $p(A)$ se contrai equivariantemente em um ponto $b_{j} \in p(A)_{j}, p^{K}\left(A^{K}\right)$ é contornável em $B^{K}$, para $(K) \in I s o(B)$.

Supondo que $f: X \rightarrow X$ é uma $G$-aplicação que preserva fibra, que para todo subfibrado $Y_{j}$ de A temos que $Y_{j}$ é um G-subconjunto fechado, localmente contrátil de $Y$ e $Y_{j}^{K}$ é contornável em $Y^{K}$, para todo $(K) \in I s o(Y)$, e que $A^{K}$ intercepta todas as $W K$-classes de pontos fixos essenciais de $f_{b_{j}}^{K}: W K\left(p^{K}\right)^{-1}\left(b_{j}\right) \rightarrow W K\left(p^{K}\right)^{-1}\left(b_{j}\right)$ para pelo menos um $b_{j}$ na componente $p^{K}\left(A^{K}\right)_{j}$, para todo $(K) \in I s o(X)$.

Se $Z$ é um $G$-subconjunto fechado e fibrado de $A$ que intercepta todas as componentes de $A$, então, existe uma G-aplicação $g: X \rightarrow X$ que é G-homotópica a $f$ por uma homotopia que preserva fibra tal que $F i x(g)=Z$ se as condições seguintes condições forem válidas:

$(C 1)_{\mathfrak{F}}$ existe uma G-homotopia parcial que preserva fibra $H_{A}:(X \times\{0\}) \cup(A \times I) \rightarrow X$ tal que $H_{A}(\bullet, 0)=f$ e $H_{A}(\bullet, 1)=i: A \hookrightarrow X($ inclusão $)$;

$(C 2)_{\mathfrak{F}}$ toda $W K$-classe de pontos fixos essencial $F$ de $f^{K}$ está conectada a $A^{K}$ por um caminho $\gamma: I \rightarrow X^{K}$ tal que $\gamma(0) \in F, \gamma(1) \in A^{K}$ e $\{\gamma(t)\}$ é homotópica à $\left\{f^{K} \circ \gamma\right\} *\left\{H_{A}^{K}(\gamma(1), t)\right\}$ com pontos extremos fixados.

Prova do Teorema 3.11: Com o Lema 3.9 podemos assumir que:

1. $A \subset F i x(f) \subset p^{-1}(p(A))$;

2. $\bar{f}: B \rightarrow B$ possui um conjunto finito $F$ de pontos fixos em $B-p(A)$; 
Seja $f_{j}=\left.f\right|_{p^{-1}\left(p(A)_{j}\right)}: p^{-1}\left(p(A)_{j}\right) \rightarrow p^{-1}\left(p(A)_{j}\right)$ uma restrição da $f$ e notemos que $p \circ f_{j}=p$.

Observamos que usando $X=p^{-1}\left(p(A)_{j}\right), \quad A=A \cap p^{-1}\left(p(A)_{j}\right), B=p(A)_{j}, \quad b_{0}=$ $b_{j}, Y_{0}=Y_{j}$ e $f=f_{j}$ temos que as hipóteses do Lema 3.10 estão satisfeitas, e com isso, encontramos uma $G$-homotopia $H_{j}: p^{-1}\left(p(A)_{j}\right) \times I \rightarrow p^{-1}\left(p(A)_{j}\right)$ que preserva fibra entre $f_{j}$ e uma $G$-aplicação $h_{j}=H_{j}(\bullet, 1)$ tal que $F i x\left(h_{j}\right)=Z_{j}$.

Seja

$$
\begin{aligned}
\widetilde{H}_{2}:(X \times\{0\}) \cup\left(p^{-1}(F \cup p(A)) \times I\right) & \rightarrow X \\
(x, t) & \mapsto \begin{cases}f(x), & \text { se } t=0 \text { ou } p(x) \in F \\
H_{j}(x, t), & \text { se } p(x) \in p(A)_{j} .\end{cases}
\end{aligned}
$$

Com a Proposição 3.4 encontramos a $G$-homotopia $H: X \times I \rightarrow X$ tal que $p(H(x, t))=$ $\bar{f} \circ p(x)$, e tomando $h=H(\bullet, 1): X \rightarrow X$ temos que $F i x(h)=Z$ e $h$ é $G$-homotópico a $f$ por uma homotopia que preserva fibra.

Uma consequência imediata do Teorema 3.11 é dada no seguinte corolário:

Corolário 3.12 Dados $G$ um grupo finito, $\mathfrak{F}=(X, p, B, Y)$ um $G$-fibrado onde $X, B$ e $Y$ são $G$-variedades suaves e compactas, $\operatorname{dim}\left(B^{K}\right) \geq 3, \operatorname{dim}\left(B^{K}\right)-\operatorname{dim}\left(B^{K}-B_{K}\right) \geq 2$, para $(K) \in I \operatorname{so}(B)$, e $\operatorname{dim}\left(Y^{K}\right) \geq 3$ e $\operatorname{dim}\left(Y^{K}\right)-\operatorname{dim}\left(Y^{K}-Y_{K}\right) \geq 2$, para $(K) \in \operatorname{Iso}(Y)$.

Seja $A$ um $G$-subconjunto não vazio, fechado e localmente contrátil de $X$ tal que $(X, A)$ é um $G$-par de fibrados para $\mathfrak{F}, p(A)$ é um $G$-subconjunto fechado de $B$, cada componente $p(A)_{j}$ de $p(A)$ se contrai equivariantemente em um ponto, $p^{K}\left(A^{K}\right)$ é contornável em $B^{K}$, para $(K) \in I s o(B)$.

Supondo que $f: X \rightarrow X$ é uma $G$-aplicação que preserva fibra, que para todo subfibrado $Y_{j}$ de $A$ temos que $Y_{j}$ é um $G$-subconjunto fechado, localmente contrátil de $Y e$ $Y_{j}^{K}$ é contornável em $Y^{K}$, para todo $(K) \in I$ so $(Y)$, e que $A^{K}$ intercepta todas as $W K$ classes de pontos fixos essenciais de $f_{b_{j}}^{K}: G p^{-1}\left(b_{j}\right) \rightarrow G p^{-1}\left(b_{j}\right)$ para pelo menos um $b_{j}$ na componente $p^{K}\left(A^{K}\right)_{j}$, para todo $(K \in I s o(X)$.

Então, existe uma $G$-aplicação $h: X \rightarrow X$ que é $G$-homotópica a $f$ por uma homotopia que preserva fibra tal que Fix $(h)=A$ se, e somente se, as condições seguintes condições forem válidas:

$(C 1)_{\mathfrak{F}}$ existe uma G-homotopia parcial que preserva fibra $H_{A}:(X \times\{0\}) \cup(A \times I) \rightarrow X$ tal que $H_{A}(\bullet, 0)=f$ e $H_{A}(\bullet, 1)=i: A \hookrightarrow X($ inclusão $)$;

$(C 2)_{\mathfrak{F}}$ toda WK-classe de pontos fixos essencial $F$ de $f^{K}$ está conectada a $A^{K}$ por um caminho $\gamma: I \rightarrow X^{K}$ tal que $\gamma(0) \in F, \gamma(1) \in A^{K}$ e $\{\gamma(t)\}$ é homotópica à $\left\{f^{K} \circ \gamma\right\} *\left\{H_{A}^{K}(\gamma(1), t)\right\}$ com pontos extremos fixados. 
Prova do Corolário 3.12: Se as condições são válidas então aplicamos o Teorema 3.11 para $Z=A$ para garantir a existência da aplicação $h$. Se existir $h$ então o Lema 3.1 garante que $(C 1)_{\mathfrak{F}}$ e $(C 2)_{\mathfrak{F}}$ são válidas. 


\section{Apêndice A}

\section{Retração Local}

Nesse apêndice vamos construir duas retrações bem particulares que usamos nos procedimentos do capítulo 2. Sejam $\mathbb{R}^{n+1}$ o espaço real $(n+1)$ dimensional e $\epsilon>0$ um número real suficientemente pequeno. Consideremos $J=\left\{(x, 0, \ldots, 0) \in \mathbb{R}^{n+1} ; 0 \leq x \leq 1\right\} \mathrm{e}$

$$
U=\left\{\left(x_{1}, \ldots, x_{n+1}\right) \in \mathbb{R}^{n+1} ; d\left(\left(x_{1}, \ldots, x_{n+1}\right), J\right) \leq \epsilon\right\}
$$

a $\epsilon$-vizinhança fechada de $J$ em $\mathbb{R}^{n+1}$. A primeira retração que construiremos será:

$$
R_{1}: U \times I \rightarrow(U \times\{0\}) \cup(J \times I)
$$

tal que $R_{1}(x, t)=(x, 0)$ se $(x, t) \in \partial(U) \times I$ e $R_{1}(x, t)=(x, t)$ se $(x, t) \in(U \times\{0\}) \cup(J \times I)$.

Seja $\left(x, x_{1}, \ldots, x_{n}, t\right) \in U \times I \subset \mathbb{R}^{n+1} \times \mathbb{R}$, com $0 \leq x \leq 1$. Tomemos o plano $\pi$ gerado pelos pontos $\left(x, x_{1}, \ldots, x_{n}, t\right),(x, 0, \ldots, 0, t)$ e $(x, 0, \ldots, 0,0)$. Então $\pi$ é dado por:

$$
\pi=\left\{\left(x, r x_{1}, \ldots, r x_{n}, s\right) \in \mathbb{R}^{n+1} \times \mathbb{R} ; r, s \in \mathbb{R}\right\} .
$$

Se $\left(\sum_{i=1}^{n} x_{i}^{2}\right)^{\frac{1}{2}}=\epsilon$ então $R_{1}\left(x, x_{1}, \ldots, x_{n}, t\right)=\left(x, x_{1}, \ldots, x_{n}, 0\right)$, pois $\left(x, x_{1}, \ldots, x_{n}\right) \in$ $\partial(U)$. Suponhamos que $q=\left(\sum_{i=1}^{n} x_{i}^{2}\right)^{\frac{1}{2}}<\epsilon$ então:

$$
R_{1}\left(x, x_{1}, \ldots, x_{n}, t\right)= \begin{cases}(x, 0, \ldots, 0,0) & \text { se } t=\frac{2 q}{\epsilon} \\ \left(x,\left(\frac{2 q-t \epsilon}{2 q-t q}\right) x_{1}, \ldots,\left(\frac{2 q-t \epsilon}{2 q-t q}\right) x_{n}, 0\right) & \text { se } t<\frac{2 q}{\epsilon} \\ \left(x, 0, \ldots, 0, \frac{t \epsilon-2 q}{\epsilon-q}\right) & \text { se } t>\frac{2 q}{\epsilon}\end{cases}
$$

Dessa forma, $R_{1}$ é contínua em $\pi$, pois se $t$ tende a $\frac{2 q}{\epsilon}$ então tanto $\frac{2 q-t \epsilon}{2 q-t q}$ quanto $\frac{t \epsilon-2 q}{\epsilon-q}$ tendem a 0 (zero) e se $q$ tende a $\epsilon$ então $\frac{2 q-t \epsilon}{2 q-t q}$ tende a 1 (um) e $\frac{2 q}{\epsilon}$ tende a 2 (dois), $2>1 \geq t$, por isso não temos que analisar $\frac{t \epsilon-2 q}{\epsilon-q}$.

Vamos construir a fórmula acima para que ela possa ser reproduzida sempre que necessário. Primeiramente, é bom que fique claro que trabalharemos somente no plano $\pi$. Queremos projetar o ponto $\left(x, x_{1}, \ldots, x_{n}, t\right)$ em $(U \times\{0\}) \cup(J \times I) \subset \mathbb{R}^{n+1} \times \mathbb{R}$, para tanto tomemos o ponto $\left(x, \frac{x_{1} \epsilon}{q}, \ldots, \frac{x_{n} \epsilon}{q}, 2\right)$. Daí, $R_{1}$ projeta o seguimento de reta:

$$
l=\left\{\left(x, r x_{1}, \ldots, r x_{n}, \frac{2 q r}{\epsilon}\right) \in \pi ; 0 \leq r \leq \frac{\epsilon}{q}(\geq 1)\right\}
$$


no ponto $(x, 0, \ldots, 0,0)$, onde $l$ é o seguimento de reta em $\pi$ que passa pelos pontos $(x, 0, \ldots, 0,0)$ quando $r=0,\left(x, x_{1}, \ldots, x_{n}, \frac{2 q}{\epsilon}\right)$ quando $r=1$ e $\left(x, \frac{x_{1} \epsilon}{q}, \ldots, \frac{x_{n} \epsilon}{q}, 2\right)$ quando $r=\frac{\epsilon}{q}$. Os pontos de $\pi$ que estão abaixo de $l$, isto é, $t \leq \frac{2 q}{\epsilon}$, são levados em $U \times\{0\}$ e os pontos de $\pi$ que estão acima de $l$, isto é, $t \geq \frac{2 q r}{\epsilon}$, são levados em $J \times I$.

A imagem de um ponto que está abaixo de $l$ é escolhida da seguinte forma: a reta em $\pi$ que passa por $\left(x, \frac{x_{1} \epsilon}{q}, \ldots, \frac{x_{n} \epsilon}{q}, 2\right)$ e pelo ponto em questão tem que interceptar $U \times\{0\}$. O ponto de interseção entre tal reta e $U \times\{0\}$ será a imagem do ponto em questão.

A imagem de um ponto que está acima de $l$ é escolhida da seguinte forma: a reta em $\pi$ que passa por $\left(x, \frac{x_{1} \epsilon}{q}, \ldots, \frac{x_{n} \epsilon}{q}, 2\right)$ e pelo ponto em questão tem que interceptar $J \times I$. O ponto de interseção entre tal reta e $J \times I$ será a imagem

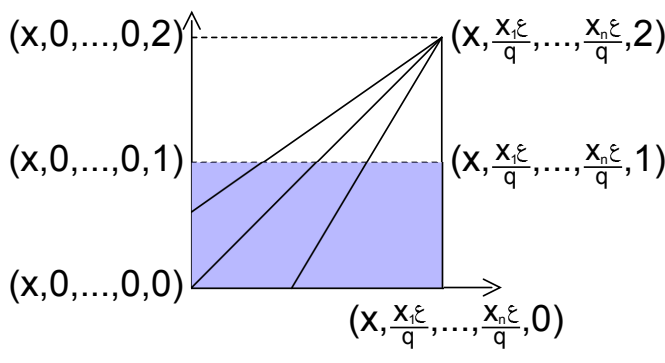

Figura A.1: Plano $\pi$ do ponto em questão.

Com o mesmo raciocínio construiremos $R_{1}$ nos pontos $\left(x_{1}, \ldots, x_{n+1}, t\right) \in U \times I \subset$ $\mathbb{R}^{n+1} \times \mathbb{R}$, com $-\epsilon \leq x_{1}<0$ e $1<x_{1} \leq 1+\epsilon$. Para construir $R_{1}$ nos pontos $\left(x_{1}, \ldots, x_{n+1}, t\right) \in U \times I \subset \mathbb{R}^{n+1} \times \mathbb{R}$, com $-\epsilon \leq x_{1}<0$, tomemos o plano $\pi$ gerado pelos pontos $\left(x_{1}, \ldots, x_{n+1}, t\right),(0, \ldots, 0, t)$ e $(0, \ldots, 0,0)$. Então $\pi$ é dado por:

$$
\pi=\left\{\left(r x_{1}, \ldots, r x_{n+1}, s\right) \in \mathbb{R}^{n+1} \times \mathbb{R} ; r, s \in \mathbb{R}\right\} .
$$

Assim, se $\left(\sum_{i=1}^{n+1} x_{i}^{2}\right)^{\frac{1}{2}}=\epsilon$ então $R_{1}\left(x_{1}, \ldots, x_{n+1}, t\right)=\left(x_{1}, \ldots, x_{n+1}, 0\right)$, pois neste caso $\left(x_{1}, \ldots, x_{n+1}\right) \in \partial(U)$. Suponhamos que $q=\left(\sum_{i=1}^{n+1} x_{i}^{2}\right)^{\frac{1}{2}}<\epsilon$ então:

$$
R_{1}\left(x_{1}, \ldots, x_{n+1}, t\right)= \begin{cases}(0, \ldots, 0,0) & \text { se } t=\frac{2 q}{\epsilon} \\ \left(\left(\frac{2 q-t \epsilon}{2 q-t q}\right) x_{1}, \ldots,\left(\frac{2 q-t \epsilon}{2 q-t q}\right) x_{n+1}, 0\right) & \text { se } t<\frac{2 q}{\epsilon} \\ \left(0, \ldots, 0, \ldots, 0, \frac{t-2 q}{\epsilon-q}\right) & \text { se } t>\frac{2 q}{\epsilon}\end{cases}
$$

Para construir $R_{1}$ nos pontos $\left(1+\delta, x_{1}, \ldots, x_{n}, t\right) \in U \times I \subset \mathbb{R}^{n+1} \times \mathbb{R}$, com $0<\delta \leq \epsilon$. Tomemos o plano $\pi$ gerado pelos pontos $\left(1+\delta, x_{1}, \ldots, x_{n}, t\right),(1,0, \ldots, 0, t)$ e $(1,0, \ldots, 0,0)$. Então $\pi$ é dado por:

$$
\pi=\left\{\left(1+r \delta, r x_{1}, \ldots, r x_{n}, s\right) \in \mathbb{R}^{n+1} \times \mathbb{R} ; r, s \in \mathbb{R}\right\} .
$$

Assim, se $\left(\delta^{2}+\sum_{i=1}^{n} x_{i}^{2}\right)^{\frac{1}{2}}=\epsilon$ então $R_{1}\left(1+\delta, x_{1}, \ldots, x_{n}, t\right)=\left(1+\delta, x_{1}, \ldots, x_{n}, 0\right)$, pois neste caso $\left(1+\delta, x_{1}, \ldots, x_{n+1}\right) \in \partial(U)$. Suponhamos que $q=\left(\delta^{2}+\sum_{i=1}^{n} x_{i}^{2}\right)^{\frac{1}{2}}<\epsilon$ então:

$$
R_{1}\left(1+\delta, x_{1}, \ldots, x_{n}, t\right)= \begin{cases}(1,0, \ldots, 0,0) & \text { se } t=\frac{2 q}{\epsilon} \\ \left(1+\left(\frac{2 q-t \epsilon}{2 q-t q}\right) \delta,\left(\frac{2 q-t \epsilon}{2 q-t q}\right) x_{1}, \ldots,\left(\frac{2 q-t \epsilon}{2 q-t q}\right) x_{n}, 0\right) & \text { se } t<\frac{2 q}{\epsilon} \\ \left(1,0, \ldots, 0, \ldots, 0, \frac{t \epsilon-2 q}{\epsilon-q}\right) & \text { se } t>\frac{2 q}{\epsilon}\end{cases}
$$


Agora, consideremos $J^{\prime}=\left\{(x, 0, \ldots, 0) \in \mathbb{R}^{n+1} ; 0 \leq x<1+\epsilon\right\}$ e $P=(1+\epsilon, 0, \ldots, 0) \in$ $\mathbb{R}^{n+1}$, a segunda retração que construiremos será:

$$
R_{2}:(U-P) \times I \rightarrow((U-P) \times\{0\}) \cup\left(J^{\prime} \times I\right)
$$

tal que $R_{2}(x, t)=(x, 0)$ se $x \in \partial(U)-P$, com $t \in I$, e $R_{2}(x, t)=(x, t)$ se $(x, t) \in$ $((U-P) \times\{0\}) \cup\left(J^{\prime} \times I\right)$.

Usando a construção feita definimos $R_{2}\left(x_{1}, \ldots, x_{n+1}, t\right)=R_{1}\left(x_{1}, \ldots, x_{n+1}, t\right)$, com $-\epsilon \leq x_{1} \leq 1$. Resta definir $R_{2}$ nos pontos da forma $\left(1+\delta, x_{1}, \ldots, x_{n}, t\right)$, com $0<\delta<\epsilon$. A construção que faremos para esses pontos será análoga a que fizemos para os pontos da forma $\left(x, x_{1}, \ldots, x_{n}, t\right)$, com $0 \leq x \leq 1$.

Seja $\left(1+\delta, x_{1}, \ldots, x_{n}, t\right) \in(U-P) \times I \subset \mathbb{R}^{n+1} \times \mathbb{R}$, com $0<\delta<\epsilon$. Tomemos o plano $\pi$ gerado pelos pontos $\left(1+\delta, x_{1}, \ldots, x_{n}, t\right),(1+\delta, 0, \ldots, 0, t)$ e $(1+\delta, 0, \ldots, 0,0)$. Então $\pi$ é dado por:

$$
\pi=\left\{\left(1+\delta, r x_{1}, \ldots, r x_{n}, s\right) \in \mathbb{R}^{n+1} \times \mathbb{R} ; r, s \in \mathbb{R}\right\} .
$$

Se $\left(\delta^{2}+\sum_{i=1}^{n} x_{i}^{2}\right)^{\frac{1}{2}}=\epsilon$ então $R_{2}\left(1+\delta, x_{1}, \ldots, x_{n}, t\right)=\left(1+\delta, x_{1}, \ldots, x_{n}, 0\right)$, pois $\left(1+\delta, x_{1}, \ldots, x_{n}\right) \in \partial(U)-P$. Suponhamos que $q=\left(\delta^{2}+\sum_{i=1}^{n} x_{i}^{2}\right)^{\frac{1}{2}}<\epsilon$ e tomemos $\Delta=\left(\epsilon^{2}-\delta^{2}\right)^{\frac{1}{2}}$ então:

$$
R_{2}\left(1+\delta, x_{1}, \ldots, x_{n}, t\right)= \begin{cases}(1+\delta, 0, \ldots, 0,0) & \text { se } t=\frac{2 q}{\Delta} \\ \left(1+\delta, x_{1}\left(\frac{2 q-t \Delta}{2 q-t q}\right), \ldots, x_{n}\left(\frac{2 q-t \Delta}{2 q-t q}\right), 0\right) & \text { se } t<\frac{2 q}{\Delta} \\ \left(1+\delta, 0, \ldots, 0, \frac{t \Delta-2 q}{\Delta-q}\right) & \text { se } t>\frac{2 q}{\Delta}\end{cases}
$$

Observamos que tanto $R_{1}$ quanto $R_{2}$ são contínuas. De fato, $R_{i}$ é contínua em $\left(x, x_{1}, \ldots, x_{n}, t\right) \in U \times I$, com $0<x<1$, por ser a composta de funções contínuas que coincidem em sua colagem, para $i=1,2$. Pelo mesmo motivo $R_{i}$ é contínua nos pontos $\left(x_{1}, \ldots, x_{n+1}, t\right) \in U \times I, \operatorname{com}-\epsilon \leq x_{1}<0$, e nos pontos $\left(1+\delta, x_{1}, \ldots, x_{n}, t\right) \in U \times I$, com $0<\delta \leq \epsilon$, para $i=1,2$.

Observamos que na colagem de $R_{i}$ nos pontos $\left(x, x_{1}, \ldots, x_{n}, t\right) \in U \times I$, com $0 \leq x \leq 1$, com os pontos $\left(x_{1}, \ldots, x_{n+1}, t\right) \in U \times I$, com $-\epsilon \leq x_{1} \leq 0$, temos que se $x>0$ tende a 0 então $R_{i}\left(x, x_{1}, \ldots, x_{n}, t\right)$ tende a

$$
\begin{cases}(0,0, \ldots, 0,0) & \text { se } t=\frac{2 q}{\epsilon} \\ \left(0,\left(\frac{2 q-t \epsilon}{2 q-t q}\right) x_{1}, \ldots,\left(\frac{2 q-t \epsilon}{2 q-t q}\right) x_{n}, 0\right) & \text { se } t<\frac{2 q}{\epsilon} \\ \left(0,0, \ldots, 0, \frac{t \epsilon-2 q}{\epsilon-q}\right) & \text { se } t>\frac{2 q}{\epsilon}\end{cases}
$$

e se $x_{1}<0$ tende a 0 então $R_{i}\left(x_{1}, \ldots, x_{n+1}, t\right)$ tende a

$$
\begin{cases}(0, \ldots, 0,0) & \text { se } t=\frac{2 q}{\epsilon} \\ \left(0,\left(\frac{2 q-t \epsilon}{2 q-t q}\right) x_{2}, \ldots,\left(\frac{2 q-t \epsilon}{2 q-t q}\right) x_{n+1}, 0\right) & \text { se } t<\frac{2 q}{\epsilon} \\ \left(0, \ldots, 0, \ldots, 0, \frac{t \epsilon-2 q}{\epsilon-q}\right) & \text { se } t>\frac{2 q}{\epsilon}\end{cases}
$$

o que mostra que $R_{i}$ é contínua em $\left(0, x_{1}, \ldots, x_{n}, t\right) \in U \times I$, com $i=1,2$. 
Na colagem de $R_{i}$ nos pontos $\left(x, x_{1}, \ldots, x_{n}, t\right) \in U \times I$, com $0<x<1$, com os pontos $\left(1+\delta, x_{1}, \ldots, x_{n}, t\right) \in U \times I$, com $0<\delta<\epsilon$, temos que se $x<1$ tende a 1 então $R_{i}\left(x, x_{1}, \ldots, x_{n}, t\right)$ tende a

$$
\begin{cases}(1,0, \ldots, 0,0) & \text { se } t=\frac{2 q}{\epsilon} \\ \left(1,\left(\frac{2 q-t \epsilon}{2 q-t q}\right) x_{1}, \ldots,\left(\frac{2 q-t \epsilon}{2 q-t q}\right) x_{n}, 0\right) & \text { se } t<\frac{2 q}{\epsilon} \\ \left(1,0, \ldots, 0, \frac{t \epsilon-2 q}{\epsilon-q}\right) & \text { se } t>\frac{2 q}{\epsilon}\end{cases}
$$

e se $1+\delta>1$ tende a 1 então $\delta$ tende a $0, \Delta$ tende a $\epsilon$ e $R_{i}\left(1+\delta, x_{1}, \ldots, x_{n}, t\right)$ tende a

$$
\begin{cases}(1,0, \ldots, 0,0) & \text { se } t=\frac{2 q}{\Delta} \\ \left(1, x_{1}\left(\frac{2 q-t \epsilon}{2 q-t q}\right), \ldots, x_{n}\left(\frac{2 q-t \epsilon}{2 q-t q}\right), 0\right) & \text { se } t<\frac{2 q}{\epsilon} \\ \left(1,0, \ldots, 0, \frac{t \epsilon-2 q}{\epsilon-q}\right) & \text { se } t>\frac{2 q}{\epsilon}\end{cases}
$$

o que mostra que $R_{i}$ é contínua em $\left(1, x_{1}, \ldots, x_{n}, t\right) \in U \times I$, com $i=1,2$. 


\section{Apêndice B}

\section{Fibrado vs Fibração}

O objetivo desse apêndice é demonstrar a proposição:

Proposição B.1 Sejam $G$ um grupo finito e $\mathfrak{F}=(X, p, B, Y)$ um G-fibrado com espaço base $B$ Hausdorff e paracompacto, então $(X, p, B)$ é uma $G$-fibração regular.

Não o fizemos na seção 3.2 por ser uma prova um tanto longa e não queríamos perder o foco do que estávamos fazendo. A prova que faremos é uma generalização do que é feito no Corolário 2.7.13 de [2] para o caso equivariante.

Para começar, tomemos $G$ um grupo de Lie compacto, uma $G$-aplicação $p: X \times B$ entre os $G$-espaços topológicos $X$ e $B$ e um $G$-subconjunto $W$ do $G$-espaço de caminhos $B^{I}$ de $B$. Definimos

$$
\tilde{W}=\{(x, w, s) \in X \times W \times I ; w(s)=p(x)\}
$$

e diremos que uma $G$-aplicação $Z: \tilde{W} \rightarrow E^{I}$ é uma $G$-aplicação de levantamento estendida sobre $W$ se $p(Z(x, w, s)(t))=w(t)$ e $Z(x, w, s)(s)=x$ e diremos que $Z$ é regular se $Z(x, p(x), s)(t)=x$ para todo $(x, p(x), s) \in \tilde{W}$ e todo $t \in I$, onde $p(x)$ é visto como o caminho constante.

Lema B.2 Sejam $G$ um grupo de Lie compacto e uma $G$-aplicação $p: X \rightarrow B$ entre os $G$-espaços topológicos $X$ e B. Então $p: X \rightarrow B$ possui uma $G$-aplicação de levantamento (regular) se, e somente se, existir alguma $G$-aplicação de levantamento estendida sobre $B^{I}$ (regular).

Prova do Lema B.2: Se $Z$ for uma $G$-aplicação de levantamento estendida sobre $B^{I}$ então definimos a $G$-aplicação de levantamento por $\Lambda(x, \gamma)(t)=Z(x, \gamma, 0)(t)$, para todo $(x, \gamma) \in \Omega_{p}$ e todo $t \in I$. Se $Z$ for regular então $\Lambda(x, p(x))(t)=Z(x, p(x), 0)(t)=x$, para todo $(x, \gamma) \in \Omega_{p}$ e todo $t \in I$, assim, $\Lambda$ é regular.

Suponhamos que $\Lambda: \Omega_{p} \rightarrow X^{I}$ seja uma $G$-aplicação de levantamento e tomemos $\gamma$ um caminho em $B^{I}$. Para cada $s \in I$ definimos os caminhos

$$
\gamma_{s}(t)=\left\{\begin{array}{cl}
\gamma(s-t) & \text { se } 0 \leq t \leq s ; \\
\gamma(0) & \text { se } s \leq t \leq 1,
\end{array} \quad \text { e } \quad \gamma^{s}(t)=\left\{\begin{array}{cl}
\gamma(s+t) & \text { se } 0 \leq t \leq 1-s \\
\gamma(1) & \text { se } 1-s \leq t \leq 1
\end{array}\right.\right.
$$


Daí, definimos a $G$-aplicação de levantamento estendida sobre $B^{I}$ por

$$
Z(x, \gamma, s)(t)= \begin{cases}\Lambda\left(x, \gamma_{s}\right)(s-t) & \text { se } 0 \leq t \leq s \\ \Lambda\left(x, \gamma^{s}\right)(t-s) & \text { se } s \leq t \leq 1\end{cases}
$$

Observamos que se $\Lambda$ for regular então para todo $s \in I$ temos que $p(x)_{s}=p(x)^{s}=p(x)$. Com isso, para todo $(x, p(x), s) \in \tilde{B}^{I}$ temos que $\Lambda\left(x, p(x)_{s}\right)(s-t)=x$ para $0 \leq t \leq s$ e $\Lambda\left(x, p(x)^{s}\right)(t-s)=x$ para $s \leq t \leq 1$, provando que $Z(x, p(x), s)(t)=x$ e que $Z$ é regular.

Dados um grupo de Lie compacto $G$ e dois $G$-espaços $E$ e $B$ dizemos que uma aplicação equivariante $p: E \rightarrow B$ é uma $G$-fibração local se existir uma cobertura $\left\{U_{\alpha}\right\}_{\alpha}$ de $B$ por abertos $G$-invariantes tal que $\left.p\right|_{p^{-1}\left(U_{\alpha}\right)}=p_{\alpha}: p^{-1}\left(U_{\alpha}\right) \rightarrow U_{\alpha}$ é uma $G$-fibração e dizemos que $p: E \rightarrow B$ é uma $G$-fibração local regular se $\left.p\right|_{p^{-1}\left(U_{\alpha}\right)}=p_{\alpha}: p^{-1}\left(U_{\alpha}\right) \rightarrow U_{\alpha}$ for uma $G$-fibração regular para cada aberto $G$-invariante da cobertura $\left\{U_{\alpha}\right\}_{\alpha}$ de $B$.

Lema B.3 Sejam $G$ um grupo de Lie compacto e $\mathfrak{F}=(X, p, B, Y)$ um $G$-fibrado, então $(X, p, B)$ é uma $G$-fibração local regular.

Prova do Lema B.3: Como $\mathfrak{F}$ é um $G$-fibrado temos que para cada $b \in B$ existe um aberto $U_{b}$ de $B$ que contém $b$ e uma trivialização local $\phi_{b}: U_{b} \times Y \rightarrow p^{-1}\left(U_{b}\right)$ tais que $p \circ \phi_{b}=\pi_{1}: U_{b} \times Y \rightarrow U_{b}$ a projeção na primeira coordenada.

Observamos que se $\pi_{2}: U_{b} \times Y \rightarrow Y$ é a projeção na segunda coordenada então $\left(p, \pi_{2} \circ \phi_{b}^{-1}\right): p^{-1}\left(U_{b}\right) \rightarrow U_{b} \times Y$ é a inversa de $\phi_{b}$. Para checar essa afirmação basta, por ser $\phi_{b}$ um homeomorfismo, verificar que $\left(p, \pi_{2} \circ \phi_{b}^{-1}\right) \circ \phi_{b}=I d_{U_{b} \times Y}$.

De fato, dado $(u, y) \in U_{b} \times Y$ temos que:

$$
\begin{aligned}
\left(p, \pi_{2} \circ \phi_{b}^{-1}\right) \circ \phi_{b}(u, y) & =\left(p, \pi_{2} \circ \phi_{b}^{-1}\right) \circ \phi_{b}(u, y) \\
& =(\underbrace{p \circ \phi_{b}}_{=\pi_{1}}(u, y), \pi_{2} \circ \underbrace{\phi_{b}^{-1} \circ \phi_{b}}_{=I d}(u, y)) \\
& =\left(\pi_{1}(u, y), \pi_{2}(u, y)\right) \\
& =(u, y) .
\end{aligned}
$$

Daí, dados um ponto $b \in B$, um $G$-espaço $Z$, uma $G$-aplicação $f: Z \rightarrow p^{-1}\left(U_{b}\right)$ e uma $G$-homotopia $F: Z \times I \rightarrow U_{b}$ tais que $p \circ f(x)=F(x, 0)$, para todo $x \in Z$,

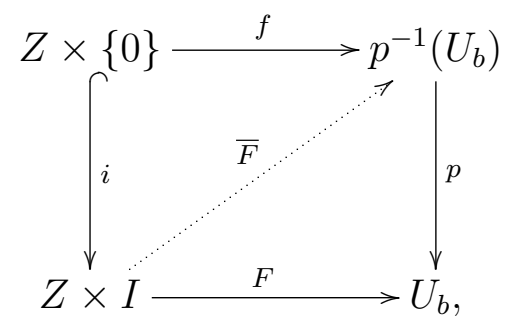

então definimos a $G$-homotopia $\bar{F}: Z \times I \rightarrow p^{-1}\left(U_{b}\right)$ por: 


$$
\begin{array}{rlccc}
Z \times I & \rightarrow & U_{b} \times Y & \stackrel{\phi_{b}}{\rightarrow} & p^{-1}\left(U_{b}\right) \\
(z, t) & \mapsto & \left(F(z, t), \pi_{2} \circ \phi_{b}^{-1} \circ f(z)\right) & \mapsto & \phi_{b}\left(F(z, t), \pi_{2} \circ \phi_{b}^{-1} \circ f(z)\right),
\end{array}
$$

onde $\pi_{2}: U_{b} \times Y \rightarrow Y$ é a projeção na segunda coordenada. Daí,

$$
p \circ \bar{F}(z, t)=\underbrace{p \circ \phi_{b}}_{=\pi_{1}}\left(F(z, t), \pi_{2} \circ \phi_{b}^{-1} \circ f(z)\right)=F(z, t)
$$

e para todo $z \in Z$ temos

$$
\begin{aligned}
\bar{F}(z, 0) & =\phi_{b}\left(F(z, 0), \pi_{2} \circ \phi_{b}^{-1} \circ f(z)\right) \\
& =\phi_{b}\left(p \circ f(z), \pi_{2} \circ \phi_{b}^{-1} \circ f(z)\right) \\
& =\underbrace{\phi_{b} \circ\left(p, \pi_{2} \circ \phi_{b}^{-1}\right)}_{=I d} \circ f(z) \\
& =f(z) .
\end{aligned}
$$

Por fim, vamos provar que essa $G$-fibração é regular. Se $z \in Z$ for tal que $F(z, 0)=$ $F(z, t)$ para todo $t \in I$ então dado $t \in I$ temos que

$$
\begin{aligned}
\bar{F}(z, t) & =\phi_{b}\left(F(z, t), \pi_{2} \circ \phi_{b}^{-1} \circ f(z)\right) \\
& =\phi_{b}\left(F(z, 0), \pi_{2} \circ \phi_{b}^{-1} \circ f(z)\right) \\
& =\bar{F}(z, 0)
\end{aligned}
$$

Dizemos que um espaço $X$ é paracompacto se para cada cobertura aberta $\left\{U_{\alpha}\right\}_{\alpha}$ de $X$ existir uma outra cobertura aberta $\left\{V_{\beta}\right\}_{\beta}$ com as seguintes propriedades:

(i) para todo $\alpha$ existe um $\beta$ tal que $V_{\beta} \subset U_{\alpha}$;

(ii) para todo ponto $x \in X$ existe um aberto $W_{x}$ de $X$ tal que $x \in W_{x}$ e $W_{x} \cap V_{\beta}=\emptyset$ a menos de uma quantidade finita de índices.

Observamos que se uma cobertura $\left\{V_{\beta}\right\}_{\beta}$ possui a propriedade (ii) então essa cobertura é dita localmente finita .

É conhecido que toda cobertura por abertos de um espaço Hausdorff paracompacto possui um refinamento enumerável. No caso de um $G$-espaço $X$ Hausdorff paracompacto, para algum grupo de Lie compacto $G$, temos que se $\left\{U_{\alpha}\right\}_{\alpha}$ é uma cobertura por abertos $G$-invariantes então existe um refinamento $\left\{\tilde{U}_{i}\right\}_{i \in \mathbb{N}}$, mas não sabemos se $\tilde{U}_{i}$ é $G$-invariante, para $i$ natural.

Contudo, temos que

$$
U_{i}=G \tilde{U}_{i}=\left\{g x \in X ; g \in G \text { e } x \in \tilde{U}_{i}\right\}
$$


é $G$ invariante e $\left\{U_{i}\right\}_{i \in \mathbb{N}}$ continua sendo um refinamento enumerável de $\left\{U_{\alpha}\right\}_{\alpha}$. Dessa forma, toda cobertura por abertos $G$-invariantes de um $G$-espaço Hausdorff paracompacto possui um refinamento $G$-invariante e enumerável.

Uma segunda observação que fazemos é que se $G$ é um grupo finito e $(E, p, B)$ uma $G$-fibração local regular com espaço base $B$ Hausdorff e paracompacto, então existe uma cobertura $\left\{\tilde{U}_{\alpha}\right\}_{\alpha}$ de $B$ por abertos $G$-invariantes tal que $\left.p\right|_{p^{-1}\left(\tilde{U}_{\alpha}\right)}=p_{\alpha}: p^{-1}\left(\tilde{U}_{\alpha}\right) \rightarrow$ $\tilde{U}_{\alpha}$ é uma $G$-fibração regular. Segue da Proposição 3.3 que existe uma $G$-aplicação de levantamento regular, $\Lambda_{\alpha}: \tilde{\Omega}_{p, \alpha} \rightarrow\left(p^{-1}\left(\tilde{U}_{\alpha}\right)\right)^{I}$, onde $\tilde{\Omega}_{p, \alpha}=\left\{(x, \gamma) \in p^{-1}\left(\tilde{U}_{\alpha}\right) \times \tilde{U}_{\alpha}^{I} ; p(x)=\right.$ $\gamma(0)\}$.

Como vimos, existe um refinamento $G$-invariante $\left\{U_{i}\right\}_{i \in \mathbb{N}}$ de $\left\{\tilde{U}_{\alpha}\right\}_{\alpha}$. Daí, dado um natural $i$ temos que existe um índice $\alpha_{i}$ tal que $U_{i} \subset \tilde{U}_{\alpha_{i}}$. Seja

$$
\Omega_{p, i}=\left\{(x, \gamma) \in p^{-1}\left(U_{i}\right) \times U_{i}^{I} ; p(x)=\gamma(0)\right\} \subset \tilde{\Omega}_{p, \alpha_{i}}
$$

e observemos que dados $(x, \gamma) \in \Omega_{p, i}$ e $t \in I$ temos que $p \circ \Lambda_{\alpha_{i}}(x, \gamma)(t)=\gamma(t) \in U_{i}$, e portanto, $\Lambda_{\alpha_{i}}(x, \gamma)(t) \in p^{-1}\left(U_{i}\right)$.

Consequentemente, $\Lambda_{i}=\left.\Lambda_{\alpha_{i}}\right|_{\Omega_{p, i}}: \Omega_{p, i} \rightarrow\left(p^{-1}\left(U_{i}\right)\right)^{I}$ é uma $G$-aplicação de levantamento regular para $\left(p^{-1}\left(U_{i}\right), p, U_{i}\right)$. Segue da Proposição 3.3 que $\left(p^{-1}\left(U_{i}\right), p, U_{i}\right)$ é uma $G$-fibração regular.

O lema de Zorn será usado no próximo resultado.

Lema B.4 Seja $G$ um grupo de Lie compacto e $(E, p, B)$ uma $G$-fibração local regular com espaço base $B$ Hausdorff e paracompacto então $(E, p, B)$ é uma $G$-fibração regular.

Prova do Lema B.4: Seja $\left\{\tilde{U}_{\alpha}\right\}_{\alpha}$ a cobertura de $B$ por abertos $G$-invariantes tal que $\left.p\right|_{p^{-1}\left(\tilde{U}_{\alpha}\right)}=p_{\alpha}: p^{-1}\left(\tilde{U}_{\alpha}\right) \rightarrow U_{\alpha}$ é uma $G$-fibração regular. Tomemos $\left\{U_{i}\right\}_{i \in \mathbb{N}}$ uma cobertura $G$-invariante de $B$ que refina $\left\{\tilde{U}_{\alpha}\right\}_{\alpha}$. Dado um subconjunto finito $T=\left\{j_{1}, \ldots, j_{k}\right\} \subset \mathbb{N}$ definimos um $G$-subconjunto do espaço dos caminhos $B^{I}$ de $B$ dado por

$$
W_{T}=\left\{w \in B^{I} ; w\left(\left[\frac{l-1}{k}, \frac{l}{k}\right]\right) \subset U_{j_{l}}, l=1, \ldots, k\right\} .
$$

Seja $\wp(\mathbb{N})$ a coleção de todos os subconjuntos finitos de $\mathbb{N}$, e dado um natural $k$ definimos $\wp_{k}(\mathbb{N})$ como a coleção de todos os subconjuntos com $k$ elementos de $\mathbb{N}$, segue que $\left\{W_{T}\right\}_{T \in \wp(\mathbb{N})}$ é uma cobertura de $B^{I}$ por abertos $G$-invariantes. Cada $T \in \wp(\mathbb{N})$ pode ser escrito da forma $T=\left\{j_{1}, \ldots, j_{k}\right\}$ para algum número natural $k$, e não estamos relacionando a ordem dos elementos de $T$ com a ordem $\leq$ de $\mathbb{N}$.

Para cada $j \in T \in \wp(\mathbb{N})$ existe uma $G$-aplicação de levantamento regular $\Lambda_{j}: \Omega_{p, j} \rightarrow$ $\left(p^{-1}\left(U_{j}\right)\right)^{I}$. Segue do Lema B.2 que existe uma $G$-aplicação de levantamento estendida regular sobre $U_{j}^{I}, Z_{j}: \tilde{U}_{j}^{I} \rightarrow\left(p^{-1}\left(U_{j}\right)\right)^{I}$, definida por

$$
Z_{j}(x, \gamma, s)(t)= \begin{cases}\Lambda_{j}\left(x, \gamma_{s}\right)(s-t) & \text { se } 0 \leq t \leq s \\ \Lambda_{j}\left(x, \gamma^{s}\right)(t-s) & \text { se } s \leq t \leq 1\end{cases}
$$


onde

$$
\tilde{U}_{j}^{I}=\left\{(x, \gamma, t) \in p^{-1}\left(U_{j}\right) \times\left(U_{j}\right)^{I} \times I ; \gamma(t)=p(x)\right\} .
$$

Com $Z_{j}$ podemos definir uma $G$-aplicação de levantamento estendida regular $Z_{T}$ : $\tilde{W}_{T} \rightarrow \bigcup_{j \in T}\left(p^{-1}\left(U_{j}\right)\right)^{I}$ sobre $W_{T}$ dada por

$$
Z_{T}(x, \gamma, s)(t)=Z_{j_{l}}\left(x_{j_{l}}, \gamma_{j_{l}}, \frac{l-1}{k}\right)(t), \text { se } \frac{l-1}{k} \leq t \leq \frac{l}{k},
$$

onde $j_{l} \in T=\left\{j_{1}, \ldots, j_{k}\right\}, \tilde{W}_{T}=\left\{(x, \gamma, t) \in\left(\bigcup_{j \in T} p^{-1}\left(U_{j}\right)\right) \times W_{T} \times I ; \gamma(t)=p(x)\right\}$,

$$
\gamma_{j_{l}}(t)=\left\{\begin{array}{cl}
\gamma\left(\frac{l-1}{k}\right) & \text { se } t \leq \frac{l-1}{k} \\
\gamma(t) & \text { se } \frac{l-1}{k} \leq t \leq \frac{l}{k} \\
\gamma\left(\frac{l}{k}\right) & \text { se } \frac{l-1}{k} \leq t
\end{array}\right.
$$

e dado $(x, \gamma, s) \in \tilde{W}_{T}$ tal que $\frac{d-1}{k} \leq s \leq \frac{d}{k}$ definimos $x_{j_{d}} \in p^{-1}\left(U_{j_{d}}\right)$ indutivamente, para $d \in\{1, \ldots, k\}$, por

$$
\begin{aligned}
x_{j_{1}} & =Z_{j_{1}}\left(x, \gamma_{j_{1}}, s\right)\left(\frac{1}{k}\right) ; \\
& \vdots \\
x_{j_{d}} & =Z_{j_{d}}\left(x, \gamma_{j_{d}}, s\right)\left(\frac{d}{k}\right) .
\end{aligned}
$$

De fato, $Z_{T}$ é regular porque dado $(x, p(x), s) \in \tilde{W}_{T}$ temos que $p(x) \in W_{T}$, e assim, $p(x) \in \cap_{j \in T} U_{j}$. Desse modo, $Z_{j}(x, p(x), s)(t)=x$, para todo $j \in T$, todo $(x, p(x), s) \in \tilde{U}_{j}^{I}$ e todo $t \in I$. $\operatorname{Logo}, Z_{T}(x, p(x), s)(t)=x$, para todo $(x, p(x), s) \in \tilde{W}_{T}$ e todo $t \in I$. Segue do Lema B.2 que $Z_{T}(\bullet, \bullet, 0)$ é uma $G$-aplicação que levanta todos os pontos $(e, \gamma) \in \Omega_{p}$ tais que $\gamma \in W_{T}$ de forma regular.

O próximo passo é um ajuste na cobertura $\left\{W_{T}\right\}_{T \in \wp(\mathbb{N})}$.

Para cada natural $j$ tomamos uma aplicação $f_{j}: B \rightarrow I$ tal que $f_{j}(b) \neq 0$ se, e somente se, $b \in U_{j}$. Com as $f_{j}$ 's definimos, para cada $T \in \wp_{k}(\mathbb{N})$ com $k$ natural, a aplicação $\tilde{f}_{T}: B^{I} \rightarrow I$ dada por

$$
\tilde{f}_{T}(\gamma)=\inf \left\{f_{j_{l}}(\gamma(t)) ; \frac{l-1}{k} \leq t \leq \frac{l}{k}, l=1, \ldots, k\right\} .
$$

Dessa forma, $\tilde{f}_{T}(\gamma) \neq 0$ se, e somente se, $\gamma \in W_{T}$.

Daí, para cada $T \in \wp_{m}(\mathbb{N})$ definimos a aplicação não negativa $f_{T}: B^{I} \rightarrow I$ dada por

$$
f_{T}(\gamma)=\inf \left\{\sup \left\{0, \tilde{f}_{T}(\gamma)-m\left(\sum_{H \in \wp^{m-1}(\mathbb{N})} \tilde{f}_{H}(\gamma)\right)\right\}, 1\right\},
$$

onde $\wp^{m}(\mathbb{N})=\bigcup_{1 \leq l \leq m} \wp_{l}(\mathbb{N})$. Desse modo, para cada $T \in \wp(\mathbb{N})$ definimos $W_{T}^{\prime}=\{\gamma \in$ $\left.B^{I} ; f_{T}(\gamma) \neq 0\right\} \subset W_{T}$. Notemos que a restrição de $Z_{T}$ para $\tilde{W}_{T}^{\prime}$ é uma $G$-aplicação de levantamento estendida sobre $W_{T}^{\prime}$ regular. 
Observamos que para cada $\gamma \in B^{I}$ existe um menor natural $m$ tal que existe um subconjunto dos naturais $T=\left\{j_{1}, \ldots, j_{m}\right\}$ formado por $m$ elementos tal que $\tilde{f}_{T}(\gamma) \neq 0$. Então $\sum_{H \in \wp^{m-1}(\mathbb{N})} \tilde{f}_{H}(\gamma)=0$ e $f_{T}(\gamma)=\tilde{f}_{T}(\gamma) \neq 0$, isto é, $\gamma \in W_{T}^{\prime}$, o que prova que $\left\{W_{T}^{\prime}\right\}_{T \in \wp(\mathbb{N})}$ cobre $B^{I}$.

Ainda pensando em $\gamma$, tomemos um natural $M>m$ tal que $\tilde{f}_{T}(\gamma)>\frac{1}{M}$. Então

$$
\sum_{H \in \wp^{M-1}(\mathbb{N})} \tilde{f}_{H}(\gamma)>\frac{1}{M} \text { e } M\left(\sum_{H \in \wp^{M-1}(\mathbb{N})} \tilde{f}_{H}(\gamma)\right)>1
$$

Dessa forma, $M\left(\sum_{H \in \wp^{M-1}(\mathbb{N})} \tilde{f}_{H}(\alpha)\right)>1$ para todo caminho $\alpha$ em alguma vizinhança de $V_{\gamma}$ de $\gamma$. Portanto, $f_{H}(\alpha)=0$ para todo $H \in \wp_{k}(\mathbb{N})$ com $k>M$ e $\alpha \in V$, e assim $W_{H}^{\prime}$ é disjunto de $V$. Consequentemente, apenas uma quantidade finita de elementos da subcoleção $\left\{W_{T}^{\prime}\right\}_{T \in\left(\wp^{M}(\mathbb{N})-\wp^{m-1}(\mathbb{N})\right)}$ intercepta $V$ e assim a coleção $\left\{W_{T}^{\prime}\right\}_{T \in \wp(\mathbb{N})}$ é localmente finita.

Nosso último passo é criar uma $G$-aplicação de levantamento regular sobre $B^{I}$ para $(E, p, B)$. Para cada $T \in \wp(\mathbb{N})$ definimos $\Omega_{p, T}=\left\{(e, \gamma) \in \Omega_{p} ; \gamma \in W_{T}^{\prime}\right\}$ e lembramos que $\Lambda_{T}=Z_{T}(\bullet, \bullet, 0)$ é uma $G$-aplicação de levantamento regular para $\Omega_{p, T}$.

Observamos que a coleção formada pelos pares $\left(T, \Lambda_{T}\right)$, com $T \in \wp(\mathbb{N})$, pode ser parcialmente ordenada pela relação: $\left(T, \Lambda_{T}\right) \preceq\left(H, \Lambda_{H}\right)$ se, e somente se, $T \subseteq H$ e $\Lambda_{T}(e, \gamma)=\Lambda_{H}(e, \gamma)$ sempre que $(e, \gamma) \in \Omega_{p, T}$.

Sejam $\left\{\left(H, \Lambda_{H}\right)\right\}_{H \in J}$ uma subcoleção totalmente ordenada por $\preceq, T=\bigcup_{H \in J} H$ e $\Omega_{p, T}=\bigcup_{H \in J} \Omega_{p, H}$

Seja $U$ um aberto de $\Omega_{p, T}$ que intercepta uma quantidade finita de $\Omega_{p, H}$ tal que $H \subset T$ e $H \in J$, digamos que $U \cap \Omega_{p, H_{j_{l}}} \neq \emptyset$ se, e só se, $l=1, \ldots, k$ e que $H_{j_{1}} \subset \cdots \subset H_{j_{k}}$. Assim, para todo $H \in J$ tal que $H_{j_{k}} \subseteq H$ e para todo $(x, \gamma) \in \Omega_{p, H_{j_{k}}}$ temos que $\Lambda_{H_{j_{k}}}(x, \gamma)=$ $\Lambda_{H}(x, \gamma)$

Portanto, para cada $(e, \gamma) \in \Omega_{p, T}$ definimos $\Lambda_{T}(e, \gamma)=\Lambda_{H}(e, \gamma)$ para algum $H \in J$ tal que $(e, \gamma) \in \Omega_{p, H}$. Observamos que $\Lambda_{T}$ é regular porque se $(x, p(x)) \in \Omega_{p, T}$ e $t \in I$ então $\Lambda_{T}(x, p(x))(t)=\Lambda_{H}(x, p(x))(t)=x$ para algum $H \in J$ tal que $(x, p(x)) \in \Omega_{p, H}$.

Segue do lema de Zorn que existe um elemento maximal $\left(M, \Lambda_{M}\right)$ para a coleção formada pelos pares $\left(T, \Lambda_{T}\right)$, com $T \in \wp(\mathbb{N})$. Para concluir vamos provar que $M=\mathbb{N}$. Suponhamos que exista $j \in \mathbb{N}-M$ e seja $\tilde{M}=M \cup\{j\}$. Definimos $h: W_{\tilde{M}}^{\prime} \rightarrow \mathbb{R}$ por $h(\gamma)=\frac{f_{M}(\gamma)}{f_{\tilde{M}}(\gamma)}, \operatorname{assim}$

$$
h(\gamma) \neq\left\{\begin{array}{lll}
0 & \Leftrightarrow & \gamma \in W_{M}^{\prime} \\
1 & \Leftrightarrow \gamma \in W_{\tilde{M}}^{\prime}
\end{array}\right.
$$

Dessa forma, definimos uma $G$-aplicação auxiliar $\mu: \Omega_{p,\{j\}} \rightarrow X$ dada por

$$
\mu(x, \gamma)= \begin{cases}x & \text { se } h(\gamma)=0 \\ \Lambda_{M}(x, \gamma)(h(\gamma)) & \text { se } h(\gamma) \neq 0\end{cases}
$$


para definir uma $G$-aplicação de levantamento regular $\Lambda_{\tilde{M}}$ para $\Omega_{p, \tilde{M}}$ dada por

$$
\Lambda_{\tilde{M}}(x, \gamma)(t)= \begin{cases}\Lambda_{M}(x, \gamma)(t) & \text { se } 0 \leq t \leq h(\gamma), h(\gamma) \neq 0 \\ Z_{M}(\mu(x, \gamma), \gamma, h(\gamma))(t) & \text { se } h(\gamma) \leq t \leq 1, h(\gamma) \neq 1\end{cases}
$$

O que é um absurdo porque $\left(M, \Lambda_{M}\right)$ é maximal e $\left(M, \Lambda_{M}\right) \preceq\left(\tilde{M}, \Lambda_{\tilde{M}}\right)$. Portanto $M=\mathbb{N}$ e segue da Proposição 3.3 que $(E, p, B)$ é uma $G$-fibração regular.

Prova da Proposição B.1: Dado o $G$-fibrado $\mathfrak{F}=(X, p, B, Y)$, segue do Lema B.3 que $(X, p, B)$ é uma $G$-fibração local regular. Daí, segue do Lema B.4 que $(X, p, B)$ é uma $G$-fibração regular. 


\section{Referências Bibliográficas}

[1] R. F. Brown. The Lefschetz Fixed Point Theorem. Scott, Foresman and Co., Glenview, I11., 1971.

[2] E. H. Spanier. Algebraic Topology. McGraw-Hill, New York, 1966.

[3] A. Dold. Lectures on Algebraic Topology. Springer-Verlag, Berlin, (1972).

[4] J. Dugundji. Topology. Boston, Allyn and Bacon, 1965.

[5] S.-T. Hu. Homotopy Theory. Academic Press, 1959.

[6] T. tom Dieck. Transformation Groups. de Gruyter, Berlin, 1987.

[7] A. Dold. Fixed point index and fixed point theorems for euclidean neighborhood retracts. Topology, 4 (1966), 1-8.

[8] B. Jiang. On the least number of fixed points. Amer. J. Math. 102 (1980) ,no.4 , 749-763.

[9] H. Schirmer. Fixed point sets in a prescribed homotopy class. Topology Appl. 37 (1990), 153-162.

[10] H. Schirmer. Fixed point sets of deformations of pairs of spaces. Topology Appl. 23 (1986), 193-205.

[11] H. Schirmer. A relative Nielsen number. Pacific J. Math. 122 (1986), 459-473.

[12] E. Fadell e P. Wong. On deforming G-maps to be fixed point free. Pacific J. Math. 132 (1988), 277-281.

[13] P. Wong. Equivariant Nielsen fixed point theory for G-maps. Pacific J. Math. 150 (1991), 179-200.

[14] P. Wong. On the location of fixed points of G-deformations. Topology Appl. 39 (1991), 159-165.

[15] P. Wong. Equivariant path fields on G-complexes. Rocky Mountain J. Math. 22 (1992), 1139-1145. 
[16] P. Wong. Equivariant Nielsen numbers. Pacific J. Math. 159 (1993), 153-175.

[17] J. Better. Nielsen fixed point theory for G-compactly fixed maps of pairs. JP J. Geom. Topol. 4 (2004), 269-293.

[18] D. Wilczyński. Fixed point free equivariant homotopy classes. Fund. Math. 123 (1984), 47-59.

[19] R. Brown e C. Soderlund. Fixed point sets of fiber-preserving maps. J. Fixed Point Theory Appl. 2 (2007), 41-53.

[20] C. Soderlund. Fixed poins sets of maps homotopic to a given map. Fixed Point Theory Appl. 2006, Article ID 46052, 20pp.

[21] Chi W. Ng. Fixed point sets of maps of pairs. Ph.D. thesis, UCLA, 1995.

[22] M. Murayama. On G-ANR's and their G-homotopy types. Osaka J. Math. 20 (1983), 479-512.

[23] S. Illman. Smooth equivariant triangulations of $G$-manifolds for $G$ a finite group. Math. Ann. 233 (1978), 199-220.

[24] G. Allaud e E. Fadell A Fiber Homotopy Extension Theorem. Trans. Amer. Math. Soc. 104 (1962), 239-251.

[25] T. N. Fomenko On The Least Number Of Fixed Points Of An Equivariant Map. Math. Notes 69, vol 1 (2001), 88-98. 


\section{Índice Remissivo}

$G$ -

ação, 15

ANR, 17

aplicação, 15

aplicação de levantamento, 19

aplicação de levantamento regular, 19

classe de pontos fixos, 18

classe de pontos fixos essencial, 18

complexo simplicial, 18

conjunto, 15

ENR, 17

espaço, 15

fibração, 19

fibração local, 77

fibrado, 19

homotopia, 15

homotopia relativa, 15

invariante, 15

módulo, 21

par fibrado, 19

par topológico, 17

retrato de uma vizinhança, 17

retrato forte de uma vizinhança, 17

subconjunto fibrado, 19

triangularização, 18

$\epsilon$-homotopia, 2

órbita, 22

indice

da $G$-classe de pontos fixos, 18

da classe de pontos fixos, 11

de pontos fixos $\left(\mathbb{S}^{n}\right), 9$

de pontos fixos (ENR), 10

ação

simplicial, 17 ação de um grupo, 15

ANR, 2

aplicação, 2

índice de pontos fixos $\left(\mathbb{S}^{n}\right), 9$

índice de pontos fixos(ENR), 10

compactamente fixada, 8

de levantamento, 8

de levantamento (equivariante), 19

de levantamento estendida, 76

de levantamento regular, 8

de levantamento regular (equivariante), 19

equivariante, 15

grau local, 9

induzida equivariante, 19

número de Nielsen, 11

preserva fibra (fibração), 7

preserva fibra (fibrado), 6

preserva filtração, 16

proximal, 5

atlas, 17

suave, 17

bordo, 2

cadeia, 16

caminho, 3

especial, 6

especialmente homotópicos, 6

PL, 5

carta, 17

classe

de conjugação, 16

de pontos fixos, 10

de pontos fixos equivariante, 18 
de pontos fixos essencial, 11

equivariante de pontos fixos essencial,

18

fundamental, 9

cobertura

localmente finita, 78

complexo simplicial, 3

equivariante, 18

conjunto de pontos fixos, 8

contornável, 3

dimensão

complexo simplicial, 3

ENR, 3

equivariantemente triangularizável, 18

espaço

dos caminhos, 3

equivariante, 15

homogêneo, 22

paracompacto, 78

extensão por equivariância, 41

face, 3

maximal, 3

fecho, 2

fibração, 7

$G$-equivalente, 19

local, 77

classe de espaços, 7

equivalente, 8

equivariante, 19

preserva fibra, 7

regular, 7,8

fibrado, 6

equivariante, 19

filtração, 16

função

coordenada, 17

de classe $C^{\infty}, 17$

simplicial, 3

suave, 17

grau local, 9 grupo

de Lie, 17

de Weyl, 16

topológico, 15

homotopia

preserva fibra, 6

equivariante, 15

especial, 8

estacionária, 8

relativa, 2

relativa equivariante, 15

interior, 2

invariante, 15

levantamento

de $G$-homotopias, 19

de homotopia, 7

localmente compacto, 3

localmente contrátil, 3

métrica $G$-invariante, 18

mergulho, 3

número de Nielsen, 11

normalizador, 15

ordem admissível, 16

p-simplexo, 3

par

G-ENR, 17

de espaços, 2

fibrado, 6

fibrado equivariante, 19

topológico equivariante, 17

paracompacto, 78

PL-caminho, 5

normal, 5

poliedro, 5

ponto de corte local, 3

preservar

fibra em fibração, 7 
fibra em fibrado, 6

fibra equivariantemente (fibração), 19

fibra equivariantemente (fibrado), 19

filtração, 16

realização geométrica

complexo simplicial, 4

simplexo, 4

representação, 21

retração, 2

retrai, 2

equivariantemente, 17

retrato

de uma vizinhança, 2

equivariante de uma vizinhança, 17

equivariante forte de uma vizinhança,

17

forte de uma vizinhança, 2

segmento, 5

simplexo, 3

maximal, 3

$\mathrm{p}, 3$

subcomplexo, 3

subconjunto fibrado, 7

equivariante, 19

subdivisão, 4

subgrupo de isotropia, 16

tipo de

órbita, 22

isotropia, 16

triangularização, 5

equivariante, 18

trivialização local, 6

vértice, 3

variedade

suave, 17

vizinhança, 2

aberta, 2

fechada, 2 